\title{
PROBLEMAS ABSTRATOS DE CAUCHY \\ EM ESPAÇOS DE BANACH
}

Doherty Andrade

TESE APRESENTADA

AO

INSTITUTO DE MATEMÁTICA E ESTATISTICA

DA

UNIVERSIDADE DE SÃO PAULO

PARA OBTENÇÃO DO GRAU DE DOUTOR

EM

MATEMÁTICA

Área de concentração: Análise

Orientador: Profa. Dra. Carmen Sílvia Cardassi

- São Paulo, janeiro de 1994 - 
A meus pais e

a todos que

amam Deus com sinceridade. 


\section{AGRADECIMENTOS}

Agradeço a todos que, mesmo de forma indireta, estiveram envolvidos neste trabalho.

Agradecimentos especiais à Profa. Dra. Carmen Sílvia Cardassi por sua orientação dedicada e competente, o que tornou possível a realização deste trabalho.

Agradeço ao Prof. Luiz Augusto F. de Oliveira pelo apoio e valiosas sugestões.

Agradeço aos amigos do IME-USP e do DMA-UEM pelo carinho e incentivo; aos Profs. Carlos Humes Jr., Vera Giusti, Roseli Fernandes, Jorge Aragona, Chaim, Iracema Bund, César Polcino, Ruy Exel, Zara, Luiz Fichimann, José Carlos, Luciano Barbanti, Antônio Pereira, Plínio Simões, Elói Galego, João Toledo, M. T. Fu, Carla Maria Fossi e Rosa Maria Tassi pelo pelo incentivo e exemplo.

Agradeço também aos meus pais e à minha família pelo carinho e apoio que foram muito importantes neste trabalho. 


\section{RESUMO}

Neste trabalho estudamos problemas abstratos de Cauchy $P A(t)(F u, x)$

$$
\left\{\begin{array}{l}
\frac{d u}{d t}+A(t) u(t) \ni(F u)(t), 0 \leq t \leq T \\
u(0)=x
\end{array}\right.
$$

onde $A(t), 0 \leq t \leq T$, é uma família de operadores acretivos sobre um espaço de Banach $X$ e $F$ é uma aplicação contínua de $C([0, T] ; X)$ em $C([0, T] ; X)$. Provamos existência de solução (segundo Pavel) local e estudamos propriedades do conjunto solução da família de problemas $P A(t) C(F u ; x), F \in N$ e $N$ com algumas propriedades. Definimos problemas normais e demonstramos que sob determinadas condições estes problemas têm solução. Provamos que sob algumas condições estes problemas têm soluções Lipschitz contínuas. Provamos existência de duas soluções não triviais para um problema elíptico com ressonância forte no infinito

$$
\left\{\begin{array}{l}
-\Delta u=\lambda_{1} u+g(x, u) \text { em } \Omega \\
u=0 \text { em } \partial \Omega
\end{array}\right.
$$

onde $\Omega$ é um domínio limitado em $\mathbf{R}^{N}$ com fronteira $\partial \Omega$ suave, $\lambda_{1}$ é o primeiro autovalor de $\left(-\Delta, H_{0}^{1}(\Omega)\right)$ e $g: \Omega \times \mathbf{R} \longrightarrow \mathbf{R}$ é uma função limitada e de Carathéodory. Não supomos condição de simetria em $g$ ou hipóteses que implicam a condição Palais-Smale $(P S)_{c}, \forall c \in \mathbf{R}$. 


\section{ABSTRACT}

In this work we study abstract Cauchy's problems

$$
\left\{\begin{array}{l}
\frac{d u}{d t}+A(t) u(t) \ni(F u)(t), 0 \leq t \leq T \\
u(0)=x
\end{array}\right.
$$

where $A(t), 0 \leq t \leq T$, is a family of accretive operators on a Banach space $X$ and $F$ is a continuous mapping of $C([0, T] ; X)$ into itself. We prove existence of local solutions and study properties of the set of solutions of the family problems $P A(t)(F u ; x), F \in N$, and $N$ with some properties. Normal problems are defined and we prove that under some conditions these problems have solutions. We prove the existence of solutions for an elliptic problem with strong resonance at infinity

$$
\left\{\begin{array}{l}
-\Delta u=\lambda_{1} u+g(x, u) \text { in } \Omega \\
u=0 \text { in } \partial \Omega
\end{array}\right.
$$

where $\Omega$ is a bounded domain in $\mathbf{R}^{N}$ with smooth boundary $\partial \Omega, \lambda_{1}$ is the first eigevalue of $\left(-\Delta, H_{0}^{1}(\Omega)\right)$ and $g: \Omega \times \mathbf{R} \longrightarrow \mathbf{R}$ is a bounded Carathéodory function. We do not assume any kind of symetry of $g$ or hypoteses that implies Palais-Smale condition $(P S)_{c} \forall c \in \mathbf{R}$. 


\section{Índice}

1 Resultados Básicos 1

1.1 Espaços de Banach . . . . . . . . . . . . . . . . . . . . . 1

1.2 Funções a valores vetoriais . . . . . . . . . . . . . . . . 4

1.3 A propriedade de Radon-Nikodym . . . . . . . . . . . . . . . . 7

1.4 Alguns teoremas importantes . . . . . . . . . . . . . . . . . . 7

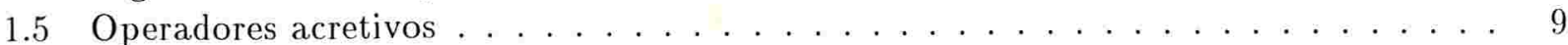

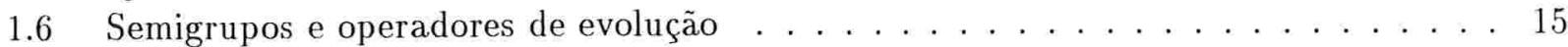

1.7 Medidas de não compacidade . . . . . . . . . . . . . . . . . . 15

2 Soluções locais de problemas abstratos de Cauchy 17

2.1 Introdução . . . . . . . . . . . . . . . . . . . . . 17

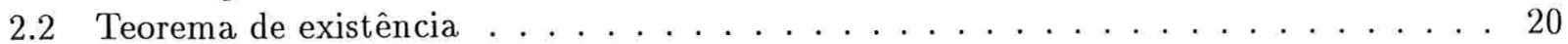

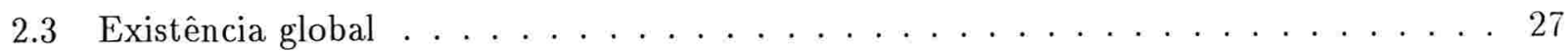

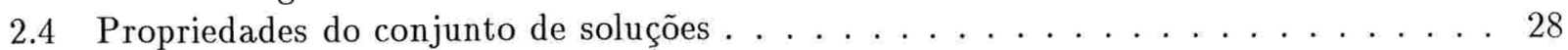

2.5 Um Resultado de convergência . . . . . . . . . . . . . . . . 35

3 Soluções Lipschitz contínuas $\quad 37$

3.1 Resultados de existência . . . . . . . . . . . . . . . . . . . . 37

3.2 Teorema de existência II . . . . . . . . . . . . . . . . . . . . 40

4 Problemas Normais $\quad 46$

4.1 Introdução . . . . . . . . . . . . . . . . . . . . . . . . 46

4.2 Aplicações . . . . . . . . . . . . . . . . . . . . . . . . 47

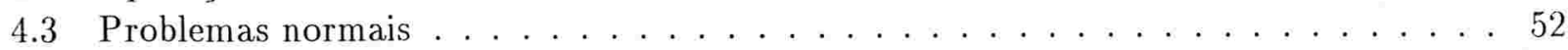

5 Solução de um problema elíptico $\quad 57$

5.1 Preliminares . . . . . . . . . . . . . . . . . . . . . 57

5.2 Resultados auxiliares . . . . . . . . . . . . . . . . . . 59

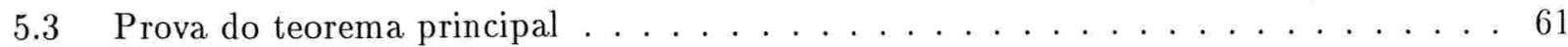




\section{INTRODUÇÃO}

O estudo de problemas de valor inicial do tipo

$$
\left\{\begin{array}{l}
\frac{d u}{d t}+A u \ni f \\
u(0)=x,
\end{array}\right.
$$

onde $A$ é um operador acretivo sobre um espaço de Banach $X$ e $f \in L^{1}(0, T ; X)$, foi basicamente iniciado com Komura [36]. Komura provou que se $X$ é um espaço de Hilbert, $A$ é $m$-acretivo, $x \in D(A)$ e $f \equiv 0$, então existe uma única função Lipschitziana $u:[0, \infty) \rightarrow D(A)$, derivável em quase toda a parte de $[0, \infty)$ e satisfazendo o problema (1) acima quase sempre. O resultado de Komura não se estende a espaços de Banach arbitrários como mostra o exemplo de Webb em [63].

Crandall e Liggett [15] demonstraram o teorema da fórmula exponencial, isto é, para $A$ acretivo sobre um espaço de Banach $X, f \equiv 0$ e $x \in \overline{D(A)}$, a sequência

$$
\left(I+\frac{t}{n} A\right)^{-n} x
$$

converge uniformemente, para $t$ em intervalos limitados de $[0, \infty)$, quando $n$ tende ao infinito. $\mathrm{Na}$ época, o significado do teorema de Crandall-Liggett não ficou claro, pois o conceito de solução mild ainda não havia sido introduzido.

Brézis e Pazzy [8] provaram que se $u$ é solução Lipschitziana de (1) em $[0, \infty)$ com $f \equiv 0$ então, (2) converge uniformemente para $u$, para $t$ em intervalos limitados de $[0, \infty)$.

Com a introdução de conceitos mais fracos de solução para (1) novos resultados de existência foram obtidos. O conceito de solução forte foi usado para definir solução fraca (não confundir com solução na topologia fraca de $X$ ) e este, por sua vez, usado por Bénilan [6] para introduzir as soluções integrais. Bénilan provou que o problema (1) com $A m$-acretivo tem sempre uma única solução integral. Sob algumas condições adicionais a solução integral é solução forte e por isso este conceito tem sido amplamente estudado e utilizado. Outros problemas mais gerais do que (1) passaram a ser abordados desde então, essencialmente de duas maneiras distintas: sob condições de compacidade ou sob condições dissipativas.

Dentre os problemas gerais estudados está o problema (3) abaixo, onde $F$ é um operador de $C([0, T] ; X)$ em si mesmo, $T>0$,

$$
\left\{\begin{array}{l}
\frac{d u}{d t}+A u(t) \ni(F u)(t), 0 \leq t \leq T \\
u(0)=x
\end{array}\right.
$$

onde $u:[0, T] \rightarrow X$ é a função desconhecida, $A$ é um operador $m$-acretivo e $x \in \overline{D(A)}$. Os problemas do tipo (3) foram estudados por Pazy [49] e por Volkmann [60], por exemplo. A existência de solução para tais problemas foi garantida sob várias condições, dentre elas, as 
condições de compacidade dos operadores $A$ e $F$ associadas a teoremas de ponto fixo. Veja por exemplo, Schechter [54] ou Gutman [25].

A maioria dos trabalhos sobre equações diferenciais em espaços de Banach usava a topologia forte de $X$. Estudos análogos relativos à topologia fraca de $X$ foram deixados de lado por muito tempo. Só recentemente problemas abstratos de Cauchy na topologia fraca de $X$ passaram a ser estudados. Szep [57] em 1971 provou um teorema tipo Peano para EDO definida num espaço de Banach reflexivo e tendo um campo de vetores fracamente contínuo. Este resultado foi estendido para espaços não-reflexivos por Cramer, Lakshimikanthamm e Mitchel [14]. Veja também Falkner [21], Knight [34] e Fitzgibbon [23].

Problemas de valor inicial do tipo (1) com o operador $A$ dependente do tempo foram objeto de estudo de Kobayashi-Kobayasi-Oharu [35] e de Pavel [48]. Pavel estendeu os conceitos de operador acretivo e solução integral demonstrou a existência de solução para equações de evolução com operador dependente do tempo.

Neste trabalho provamos resultados de existência de diversos tipos de solução para problemas do tipo (3) com o operador dependente do tempo. Definimos problemas normais, que contém os anteriores, e provamos também a existência de solução sob algumas condições. Provamos ainda a existência de duas soluções não triviais de um problema elíptico com ressonância forte. Exemplos e aplicações estão distribuídos em todo o texto. Os resultados que são conhecidos ou que são extensões de outros estão devidamente identificados com nome ou referência.

Este trabalho está dividido em cinco capítulos. No primeiro estão os principais resultados que serão utilizados mais adiante no texto e serve para introduzir as notações usadas.

No Capítulo 2 provamos a existência de solução para o problema $P A(t)(F u ; x)$

$$
\left\{\begin{array}{l}
\frac{d u}{d t}+A(t) u(t) \ni(F u)(t), 0 \leq t \leq T \\
u(0)=x
\end{array}\right.
$$

damos alguns exemplos e estudamos propriedades do conjunto solução de uma família de problemas do tipo (4). Neste capítulo provamos que se a família de operadores $A(t), 0 \leq$ $t \leq T$, gera um operador de evolução compacto então o conjunto de soluções integrais é relativamente compacto. Os resultados deste capítulo são originais.

No Capítulo 3 estudamos em que condições as soluções obtidas do teorema de existência do Capítulo 2 são Lipschitz contínuas. Resultados para um caso particular foram obtidos sob a hipótese de que a família de operadores $A(t), 0 \leq t \leq T$, seja constante independente de $t$. O teorema 3.11 é novo e generaliza um resultado de Fitzgibbon [23].

No Capítulo 4 estudamos algumas propriedades do operador de Bénilan-Pavel, $B_{x}$, e existência de solução para problemas normais, isto é, problemas da forma

$$
\left\{\begin{array}{l}
\frac{d u}{d t}+A(t) u(t) \bar{\ni}(F u)(t) \\
u(0)=x \in \overline{D(A(0))}
\end{array}\right.
$$

onde $F$ aplica $C([0,1] ; X)$ nas partes de $C([0,1] ; X)$. Os problemas normais são uma extensão de (4). O problema (5) é interpretado da seguinte forma: encontrar $u:[0,1] \rightarrow X$ contínua 
que seja solução integral de

$$
\left\{\begin{array}{l}
\frac{d u}{d t}+A(t) u(t) \ni f(t) \\
u(0)=x \in \overline{D(A(0))}
\end{array}\right.
$$

para alguma $f \in F u$. Provamos que sob determinadas condições estes problemas admitem solução. Os resultados deste capítulo também são originais.

No Capítulo 5 provamos a existência de duas soluções não triviais de um problema elíptico semi-linear com ressonância forte no infinito. Mais precisamente, mostramos que

$$
\left\{\begin{array}{l}
-\Delta u=\lambda_{1} u+g(x, u) \text { em } \Omega \\
u=0 \text { em } \partial \Omega
\end{array}\right.
$$

onde $\Omega$ é um domínio limitado em $\mathbf{R}^{N}$ com fronteira $\partial \Omega$ suave, $\lambda_{1}$ é o primeiro autovalor de $\left(-\Delta, H_{0}^{1}(\Omega)\right)$ e $g: \Omega \times \mathbf{R} \longrightarrow \mathbf{R}$ é uma função limitada e de Carathéodory, admite pelo menos duas soluções não triviais. Não fazemos hipótese alguma de simetria em $g$ e não admitimos a condição $(P S)_{c}, \forall c \in \mathbf{R}$, para o funcional associado ao problema. Estamos portanto numa situação limite. Neste capítulo utilizamos teorema de deformação, teorema de linking e métodos minimax. O teorema principal é original enquanto que para o lema 5.7 damos uma prova diferente de um caso particular de um resultado devido a Silva [55]. 


\section{Capítulo 1}

\section{Resultados Básicos}

Este capítulo tem por objetivo listar alguns resultados que serão úteis nos capítulos seguintes bem como fixar notações.

\subsection{Espaços de Banach}

Definição 1.1 Seja $X$ um espaço vetorial sobre um corpo $K$. Dizemos que $X$ é um espaço de Banach se existe uma norma

$$
x \mapsto\|x\|
$$

tal que toda sequência de Cauchy de elementos de $X$ é uma sequência convergente. Em outras palavras, $X$ munido da métrica

$$
d(x, y)=\|x-y\|
$$

induzida pela norma é espaço métrico completo.

Exceto por menção explícita em contrário, todos os espaços de Banach considerados neste trabalho serão reais e separáveis. Se $X$ é um espaço de Banach representaremos por $X^{*}$ o dual topológico de $X$, isto é,

$$
X^{*}=\left\{x^{*}: X \rightarrow \mathbf{R}: x^{*} \text { é linear e contínua }\right\} .
$$

O valor de $x^{*} \in X^{*}$ em $x \in X$ será indicado por $\left(x^{*}, x\right), x^{*}(x)$ ou $\left\langle x^{*}, x\right\rangle$. A aplicação

$$
X^{*} \ni x^{*} \mapsto\left\|x^{*}\right\|=\operatorname{Sup}\left\{\left(x^{*}, x\right): x \in X \text { e }\|x\| \leq 1\right\}
$$

torna $X^{*}$ um espaço Banach.

Além da topologia forte em $X$, isto é a topologia dada pela norma de $X$, consideraremos as topologias fraca e fraca-estrela, conforme passamos a descrever.

Definição 1.2 A topologia fraca sobre $X$, denotada por $w$-topologia, $\sigma\left(X, X^{*}\right)$ ou $\sigma_{w}$, é a menos fina das topologias de $X$ que torna todos os elementos $x^{*} \in X^{*}$ contínuos.

Todo aberto da topologia fraca é um aberto da topologia forte. O espaço $\left(X, \sigma_{w}\right)$ é um espaço de Hausdorff. 
Proposição 1.3 São equivalentes:

1) $x_{\alpha} \stackrel{\sigma_{u}}{\longrightarrow} x_{0}$,

2) $x^{*}\left(x_{\alpha}\right) \rightarrow x^{*}\left(x_{0}\right)$, para todo $x^{*} \in X^{*}$.

Para uma demonstração, veja [5], [20] ou [29].

Definição 1.4 A topologia fraca-estrela de $X^{*}$, denotada por $w^{*}, \sigma_{w^{*}}$ ou $\sigma\left(X^{*}, X\right)$, é a menos fina das topologias que torna todos os elementos $\hat{x}$ contínuos, onde

$$
X^{*} \ni x^{*} \stackrel{\hat{x}}{\rightarrow} x^{*}(x) \in \mathbf{R}
$$

para cada $x \in X$.

Proposição 1.5 São equivalentes:

1) $x_{\alpha}^{*} \stackrel{\sigma_{w^{*}}}{\rightarrow} x_{0}^{*}$

2) $x_{\alpha}^{*}(x) \rightarrow x_{0}^{*}(x)$, para todo $x \in X$.

Veja [5] ou [29] para outras informações.

Definição 1.6 Dizemos que um espaço de Banach $X$ é reflexivo se $J(X)=X$, onde $J$ é a inclusấo canônica de $X$ em $X^{* *}$, isto é,

$$
X \in x \mapsto J(x)=\hat{x} \in X^{* *} .
$$

Definição 1.7 1) Uma função $f: X \rightarrow(-\infty, \infty]$ é semicontínua inferiormente, abreviadamente sci, no ponto $x_{0}$ se,

$$
f\left(x_{0}\right) \leq \liminf _{x \rightarrow x_{0}} f(x) .
$$

Dizemos que $f$ é sci se $f$ for sci em cada ponto de $X$.

$O$ dominio efetivo de $f$ é definido por

$$
\operatorname{dom}(f)=\{x \in X: f(x)<\infty\} .
$$

e $f$ é dita própria se dom $(f)$ é não vazio.

2) $f: X \rightarrow[-\infty, \infty]$ é convexa se para cada $\lambda \in[0,1]$ vale a desigualdade

$$
f(\lambda x+(1-\lambda) y) \leq \lambda f(x)+(1-\lambda) f(y), x, y \in X,
$$

sempre que o lado direito fizer sentido. Adotamos as seguintes convenções: $( \pm r) \cdot \infty= \pm \infty$, se $r>0$ e $r \pm \infty= \pm \infty, \forall r \in \mathbf{R}$.

Lembramos que o epigráfico de uma função $f: X \rightarrow(-\infty, \infty]$ é definido por

$$
\operatorname{epi}(f)=\{(x, r) \in X \times \mathbf{R}: r \geq f(x)\} .
$$

Proposição 1.8 1) Para que $f$ seja sci é necessário e suficiente que o epigráfico de $f$ seja fechado em $X \times \mathbf{R}$.

2) Para que $f$ seja convexa é necessário e suficiente que seu epigráfico seja convexo.

3) Seja $f: X \rightarrow(-\infty,+\infty]$ convexa. Se $f$ é sci, então $f$ é sci na topologia fraca de $X$. Também $f$ é contínua no interior de $\operatorname{dom}(f)$. 
Paras as provas e outros detalhes veja Phelps [50].

Teorema 1.9 Sejam $X$ espaço de Banach reflexivo, $C \subset X$ conjunto convexo e fechado $e$ $f: C \rightarrow(-\infty,+\infty]$ uma função convexa, própria e sci tal que

$$
\lim _{\|x\| \rightarrow \infty} f(x)=+\infty
$$

caso $C$ seja ilimitado. Então, $f$ tem um mínimo em $C$, isto é, existe $x_{0} \in C$ tal que

$$
f\left(x_{0}\right)=\inf \{f(x): x \in C\} .
$$

A demonstaração pode ser encontrada em [20] ou [5].

Definição 1.10 Seja $f: X \rightarrow(-\infty,+\infty]$ uma função. Para cada $x_{0} \in \operatorname{dom}(f)$, indicamos por $\partial f\left(x_{0}\right)$ o conjunto

$$
\partial f\left(x_{0}\right)=\left\{x^{*} \in X^{*}:\left(x^{*}, y-x_{0}\right) \leq f(y)-f\left(x_{0}\right), \forall y \in \operatorname{dom}(f)\right\} .
$$

Este conjunto é denominado o subdiferencial de $f$ em $x_{0}$.

Uma consequência do teorema de Hahn-Banach é que toda função convexa, própria e semicontínua inferior tem sempre subdiferencial não vazio em todo ponto do seu domínio efetivo.

Definição 1.11 Sejam $X$ e $Y$ espaços de Banach e $\varphi: X \rightarrow Y$ uma função. Dizemos que $\varphi$ é Gateaux-diferenciável em $x \in X$ se existe uma aplicação linear contínua $\varphi^{\prime}(x): X \rightarrow Y$ tal que

$$
\lim _{h \rightarrow 0} \frac{\varphi(x+h y)-\varphi(x)}{h}=\varphi^{\prime}(x) y, \forall y \in X .
$$

A demonstração do próximo teorema pode ser encontrada em [20] ou [50].

Teorema 1.12 Seja $f: X \rightarrow(-\infty,+\infty]$ uma função convexa e própria. Se $f$ é Gateaux diferenciável no ponto $x \in \operatorname{dom}(f)$, então

$$
\partial f(x)=\left\{f^{\prime}(x)\right\}
$$

Definição 1.13 Dizemos que $F: X \rightarrow Y$ é Fréchet diferenciável no ponto $x \in X$ se existe uma aplicação linear contínua $d F(x): X \rightarrow Y$ tal que para todo $y \in X$

$$
F(x+y)-F(x)=d F(x) y+\omega(x, y)
$$

onde $\lim _{\|y\| \rightarrow 0} \frac{\omega(x, y)}{\|y\|}=0, \forall y \neq 0$.

Exemplo 1.14 Se $\varphi(x)=\|x\|$, então

$$
\partial \varphi(x)=\left\{x^{*} \in X^{*}: x^{*}(x)=\|x\| e\left\|x^{*}\right\|=1\right\} .
$$


Exemplo 1.15 Se $\phi(x)=\frac{\|x\|^{2}}{2}$, então

$$
\partial \phi(x)=J(x)=\left\{x^{*} \in X^{*}: x^{*}(x)=\|x\|^{2}=\left\|x^{*}\right\|^{2}\right\} .
$$

A aplicação J definida anteriormente é a aplicação de dualidade.

Definição 1.16 Um espaço de Banach $X$ é chamado de suave se para todo $x \in X$ com $\|x\|=1, J(x)$ tem um único elemento.

Teorema 1.17 Seja X um espaço de Banach. São equivalentes:

1) $X$ é suave.

2) A norma de $X$ é Gateaux diferenciável fora da origem.

Um espaço suave é também conhecido como espaço Gateaux suave. Veja [5] ou [3].

\subsection{Funções a valores vetoriais}

Sejam $X$ um espaço de Banach e $T$ um real positivo. Resumiremos nesta secção alguns resultados sobre funções definidas em $[0, T]$ com valores em $X$.

Definição 1.18 Uma função $f:[0, T] \rightarrow X$ é absolutamente contínua se para cada $\varepsilon>0$ existe um $\delta(\varepsilon)>0$ tal que

$$
\sum_{n=1}^{N}\left\|f\left(\beta_{n}\right)-f\left(\alpha_{n}\right)\right\| \leq \varepsilon
$$

sempre que

$$
\sum_{n=1}^{N}\left|\beta_{n}-\alpha_{n}\right| \leq \delta(\varepsilon),
$$

onde $\left(\alpha_{n}, \beta_{n}\right)$ são intervalos arbitrários de $[0, T]$ e dois a dois disjuntos.

Sabemos que funções absolutamente contínuas com valores em $X=\mathbf{R}$ são diferenciáveis quase sempre em $(0, T)$, mas este resultado não se estende para espaços de Banach quaisquer. Veja exemplo em Barbu [3]. De fato, isto caracteriza os espaços $X$ que tenham a propriedade de Radon-Nikodym.

Definição 1.19 Lembramos que uma função $f: X \rightarrow Y$ é fraco-fraco-contínua se é contínua com $X$ e $Y$ munidos da topologia fraca.

Uma função $f:[0, T] \rightarrow X$ é fraco-diferenciável em $t_{0} \in(0, T)$ se existe um elemento $f^{\prime}\left(t_{0}\right) \in X$ tal que

$$
\lim _{\delta \rightarrow 0}\left|x^{*}\left(\frac{f\left(t_{0}+\delta\right)-f\left(t_{0}\right)}{\delta}\right)-x^{*}\left(f^{\prime}\left(t_{0}\right)\right)\right|=0,
$$

para cada $x^{*} \in X^{*}$. 
Analogamente definimos função fraco-absolutamente contínua em $[0, T]$.

Indicaremos por $\mathcal{D}(0, T)$ o espaço das funções de $[0, T]$ em $\mathbf{R}$ que são infinitamente diferenciáveis em $[0, T]$ e que têm suporte compacto em $(0, T)$. Por $\mathcal{D}^{\prime}(0, T ; X)$ entendemos o espaço de todos os operadores lineares contínuos de $\mathcal{D}(0, T)$ em $X$, isto é, o espaço das distribuições de $(0, T)$ com valores em $X$. Se $f \in \mathcal{D}^{\prime}(0, T ; X)$, definimos como é usual,

$$
D^{k} f(\varphi)=(-1)^{k} f\left(D^{k} \varphi\right)
$$

para toda $\varphi \in \mathcal{D}(0, T), k \in \mathrm{N}$. $D^{k} f$ é a distribuição derivada de ordem $k$ de $f$.

Lembramos que uma função $f:[0, T] \rightarrow X$ é fortemente mensurável se existe uma sequência de funções simples mensuráveis $\left(f_{n}\right)$ tal que

$$
\lim _{n \rightarrow \infty}\left\|f_{n}(t)-f(t)\right\|=0
$$

para quase todo $t \in[0, T]$.

Para $1 \leq p \leq \infty, L^{p}(0, T ; X)$ indicará o espaço das funções $f$ definidas quase sempre em $(0, T)$ a valores em $X$, fortemente mensuráveis tais que $\|f(.)\|^{p}$ é Lebesgue integrável em $[0, T]$. Funções de $L^{p}(0, T ; X)$ são chamadas funções $p$-Bochner integráveis. Se introduzirmos em $L^{p}(0, T ; X)$ a norma

$$
\|f\|_{p}=\left(\int_{0}^{T}\|f(t)\|^{p} d t\right)^{1 / p}
$$

$1 \leq p<\infty \mathrm{e}$,

$$
\|f\|_{\infty}=\operatorname{ess} \sup \{\|f(t)\| ; 0 \leq t \leq T\},
$$

se $p=\infty$, então $L^{p}(0, T ; X)$ é um espaço de Banach.

Se $X$ é reflexivo e se $1<p<\infty$, então $L^{p}(0, T ; X)$ é reflexivo; e se $X$ tem a propriedade de Radon-Nikodym, então $L^{p}(0, T ; X)$ também tem esta propriedade.

O espaço $L^{1}(0, T ; X)$, como no caso de dimensão finita, pode ser identificado com um subconjunto de $\mathcal{D}^{\prime}(0, T ; X)$. Por $W^{k, p}(0, T ; X)$, onde $k$ é natural e $1 \leq p \leq \infty$, entendemos o espaço das distribuiçôes vetoriais que satisfazem

$$
D^{i} f \in L^{p}(0, T ; X), 0 \leq i \leq k,
$$

onde $D^{i}$ é a derivada no sentido de distribuições .

Denotamos por $A^{k, p}(0, T ; X), k$ natural e $1 \leq p \leq \infty$, o espaço das funções $f:[0, T] \rightarrow X$ absolutamente contínuas cujas derivadas $\frac{d^{i} f}{d t^{i}}$, definidas quase sempre são absolutamente contínuas para $1 \leq i \leq k-1$ e pertencem a $L^{p}(0, T ; X)$.

A relação entre $W^{k, p}(0, T ; X)$ e $A^{k, p}(0, T ; X)$ é dada pelo seguinte resultado, cuja demonstração pode ser encontrada [3] ou [8].

Teorema 1.20 Sejam $X$ um espaço de Banach e $f \in L^{p}(0, T ; X), 1 \leq p \leq \infty$. São equivalentes:

1) $f \in W^{k, p}(0, T ; X)$

2) Existe $g \in A^{k, p}(0, T ; X)$ tal que $f(t)=g(t)$ quase sempre em $(0, T)$. 
Definição 1.21 Dizemos que $f:[0, T] \rightarrow X$ é de variação limitada se existe uma constante $C$ tal que para toda subdivisão

$$
0=a_{0}<\ldots<a_{n}=T
$$

tem-se

$$
\sum_{k=1}^{n}\left\|f\left(a_{k}\right)-f\left(a_{k-1}\right)\right\| \leq C .
$$

$\dot{A}$ menor constante $C$ verificando a relação acima chamamos variação total de $f$ em $[0, T]$. É comum chamar uma função de variação limitada, segundo esta definição, de funçâa de variaçâo limitada forte.

Uma função $f:[0, T] \rightarrow X$ de variação limitada (forte) tem limites laterais em quase todo ponto e é contínua exceto para um conjunto enumerável de descontinuidades de primeiro tipo.

Teorema 1.22 Se $f:[0, T] \rightarrow X$ é de variação limitada forte e fraca-diferenciável quase sempre e com derivada $g$, então $g \in L^{1}(0, T ; X)$. Se $f$ é também fraca-absolutamente contínua, entâo ela pode ser representada como uma integral indefinida de $g$, isto é,

$$
f(x)-f(0)=\int_{0}^{x} g(\sigma) d \sigma,
$$

para todo $x \in[0, T]$.

Veja Hille-Phillips [28] para uma demonstração.

Os seguintes resultados relacionando funções absolutamente contínuas e funções de variação limitada serão também úteis.

Definição 1.23 Denotamos por $L_{l o c}^{p}(0, T ; X)$ o espaço das funções fortemente mensuáveis $f:(0, T) \rightarrow X$ tal que $f \in L^{p}(0, k ; X)$ para todo $0<k<T$.

Teorema 1.24 1) Seja $f \in L_{\text {loc }}^{1}(0, T ; X)$. Então são equivalentes:

a) $\frac{d f}{d t} \in L_{l o c}^{1}(0, T ; X)$

b) Existe $f_{1}:[0, T] \rightarrow X$ contínua em $[0, T]$, derivável em $(0, T)$ com $f_{1}^{\prime} \in L_{\text {loc }}^{1}$ e $f=f_{1}$ quase sempre.

2) Para $f:[0, T] \rightarrow X$ são equivalentes:

a) $f$ é absolutamente continua.

b) $f$ é de variação limitada e $V_{f}$ é absolutamente contínua, onde $V_{f}(t)$ é igual à variação total de $f$ em $[0, t]$.

c) Existe $g \in L^{1}(0, T ; \mathbf{R})$ tal que

$$
\lim _{h \rightarrow 0^{+}} \int_{0}^{T-h}\left|\left\|\frac{f(t+h)-f(t)}{h}\right\|-g(t)\right| d t=0
$$

3) Seja $f \in C([0, T] ; X)$. São equivalentes:

a) $\frac{d f}{d t} \in L^{1}(0, T ; X)$

b) f é absolutamente contínua em $[0, T]$ e diferenciável para quase todo $t \in[0, T]$.

As demonstrações podem ser encontradas em [40]. 


\subsection{A propriedade de Radon-Nikodym}

Definição 1.25 Seja $(\Omega, \Sigma, \mu)$ um espaço de medida finita. Um espaço de Banach $X$ tem a propriedade de Radon-Nikodym (RNP) com relação a $(\Omega, \Sigma, \mu)$ se para cada medida vetorial $G: \Sigma \rightarrow X$ de variaçấo limitada e $\mu$-contínua existe ua função função $g \in L^{1}(\Omega, \Sigma, \mu ; X)$ tal que

$$
G(E)=\int_{E} g d \mu, \forall E \in \Sigma .
$$

Um espaço $X$ tem $R N P$ se $X$ tem $R N P$ com relaçâa a todo espaço de medida finita $(\Omega, \Sigma, \mu)$.

Lembramos que $L^{p}(\Omega, \Sigma, \mu ; X), 1 \leq p \leq \infty$, é definido de modo inteiramente análogo a $L^{p}(0, T ; X)$ da seç̧ão anterior. Para uma medida vetorial $F$, a variação de $F$, é a função não negativa $|F|: \Sigma \rightarrow[0, \infty]$ definida por

$$
|F|(E)=\sup _{\Pi} \sum_{A \in \Pi}\|F(A)\|,
$$

onde o supremo é tomado sobre todas as partições $\Pi$ de $E$ em número finito de elementos disjuntos de $\Sigma$. Se $|F|(\Omega)<\infty$, diremos que $F$ tem variação limitada.

Dizemos que a medida vetorial $F$ é $\mu$-contínua se

$$
\lim _{\mu(E) \rightarrow 0} F(E)=0 .
$$

Neste caso escrevemos $F \ll \mu$.

O próximo resultado mostra a importância dos espaços de Banach com a propriedade de Radon-Nikodym.

Teorema 1.26 Seja $X$ um espaço de Banach. São equivalentes:

1) $X$ tem a $R N P$,

2) Toda $f:[0,1] \rightarrow X$ absolutamente contínua é diferenciável em quase todo $t \in[0,1]$.

3) Toda $f:[0,1] \rightarrow X$ de variação limitada é diferenciável quase sempre em $[0,1]$.

Mais sobre medidas vetoriais e propriedade de Radon-Nikodym pode ser encontrado no livro "Vector Measures" de Diestel e Uhl [19].

\subsection{Alguns teoremas importantes}

Definição 1.27 Sejam $(\Omega, \Sigma, \mu)$ espaço de medida $\sigma$-finita e $K \subset L^{1}(\Omega, \Sigma, \mu ; X)$. Dizemos que $K$ é uniformemente integrável se para qualquer $\varepsilon>0$ existir $\delta(\varepsilon)=\delta>0$ tal que se $E \in \Sigma$ e $\mu(E)<\delta$, então

$$
\int_{E}\|f(\omega)\| \mu(d \omega)<\varepsilon, \forall f \in K .
$$


Definição 1.28 Uma família $K \subset C([0, T] ; X)$ é equicontínua se para todo $\varepsilon>0$ e todo $t_{0} \in[0, T]$ existe uma vizinhança $U$ de $t_{0}$ tal que $\left\|f(t)-f\left(t_{0}\right)\right\|<\varepsilon$ para todo $t \in U$ e para toda $f \in K$.

A família $K$ é uniformemente equicontínua se, para cada $\varepsilon>0$ existir $\delta>0$ tal que $|t-s|<\delta, t, s \in[0, T]$, implica que

$$
\|f(t)-f(s)\|<\varepsilon, \forall f \in K .
$$

A família é fraco-equicontínua se dado $\varepsilon>0, x^{*} \in X^{*}$, existe um $\delta=\delta\left(x^{*}, \varepsilon\right)>0$ tal que

$$
\left|x^{*}(x(t)-x(s))\right|<\varepsilon
$$

se $|t-s|<\delta$ para todo $x$ da família.

Teorema 1.29 (Dunford) Sejam $(\Omega, \Sigma, \mu)$ espaço de medida finita e $X$ espaço de Banach tal que $X$ e $X^{*}$ tenham $R N P$. Um subconjunto $K \subset L^{1}(\Omega, \Sigma, \mu ; X)$ é relativamente fraco compacto se:

1) $K$ é limitado.

2) $K$ é uniformemente integrável.

3) Para cada $E \in \Sigma$, o conjunto

$$
\left\{\int_{E} f d \mu ; f \in K\right\}
$$

é relativamente fraco compacto em $X$.

Para uma demonstração veja [19].

Teorema 1.30 (Ascoli-Arzelá) Um subconjunto $K \subset C([0, T] ; X)$ é relativamente (sequencialmente) compacto se, e somente se, $K$ é equicontínuo em $[0, T]$ e toda secção

$$
K(t)=\{f(t) ; f \in K\}
$$

é relativamente compacto.

Teorema 1.31 (Ascoli-Arzelá-fraco) Um subconjunto $K \subset C([0, T] ; X)$ é relativamente sequencialmente fraco compacto se, e somente se, $K$ é fraco equicontínuo em $[0, T]$ e toda seção $K(t)$ é relativamente fraco-compacto em $X$.

Veja [37] e [19] para as demonstrações.

Teorema 1.32 (Ponto Fixo de Schauder) Seja $C$ subconjunto convexo, limitado e fechado de um espaço Banach $X$. Se $T: C \rightarrow X$ é função compacta com $T(C) \subset C$, entâa existe $x \in C$ tal que $T(x)=x$.

Veja [31] ou [29]. 
Teorema 1.33 Sejam $(M, d)$ um espaço métrico completo, $T: M \rightarrow M$ uma aplicação e $\varphi$ uma função real definida sobre a imagem $P$ da métrica d tal que em $\bar{P}-\{0\}$ vale:

1) $\varphi(t)<t$

2) $d(T x, T y)<\varphi(d(x, y))$

3) $\varphi$ é semicontínua superior.

Então, T tem um único ponto fixo.

Uma aplicação $T: M \rightarrow M$, onde $M$ é um espaço métrico completo, é uma contração de Rakotch se existe uma função $\alpha(t)<1$ decrescente, tal que para todo $x, y \in M$, tem-se

$$
d(T x, T y)<\alpha(d(x, y)) d(x, y) .
$$

Estas contrações satisfazem às condições do teorema acima. Para demonstração veja [31].

Teorema 1.34 Sejam $(M, d)$ um espaço métrico completo, $T: M \rightarrow M$ contínua e $\varphi$ : $M \rightarrow \mathbf{R}^{+}$semicontinua inferior satisfazendo

$$
d(x, T x) \leq \varphi(x)-\varphi(T x), \forall x \in M .
$$

Então, T tem um ponto fixo.

Qualquer contração forte satisfaz à condição do teorema 1.34. Veja [31] para as provas.

Teorema 1.35 [Nadler] Seja $(X, d)$ um espaço métrico completo e seja $f: X \rightarrow B C(X)$ uma contração. Então, $f$ tem um ponto fixo.

\subsection{Operadores acretivos}

Sejam $X$ um espaço de Banach com norma $\|$. $\|$ e $x, y \in X$. Definimos

$$
[x, y]_{h}=\frac{1}{h}[\|x+h y\|-\|x\|] .
$$

Sendo $[x, y]_{+}=\lim _{h \bigcup_{0}}[x, y]_{h}$ e $[x, y]_{-}=\lim _{h \nearrow 0}[x, y]_{h}$, temos os seguinte resultados que decorrem da convexidade da norma de $X$. Abreviaremos semicontinuidade superior e inferior por scs e sci, respectivamente.

Lema 1.36 1) Para cada par de pontos $x$ e $y$ de $X$ os limites $[x, y]_{+} e[x, y]_{-}$sempre existem. 2) $[x, y]_{+}$é scs e $[x, y]_{-}$é sci.

3) Se $x:[0, T] \rightarrow X$ tem as derivadas laterais $x_{+}^{\prime}(t)$ e $x_{-}^{\prime}(t)$, para algum $t \in(0, T)$, entâo $m(t)=\|x(t)\|$ tem derivadas laterais $e$

$$
\begin{aligned}
m_{+}^{\prime}(t) & =\left[x(t), x_{+}^{\prime}(t)\right]_{+} \\
m_{-}^{\prime}(t) & =\left[x(t), x_{-}^{\prime}(t)\right]_{-}
\end{aligned}
$$


Veja [38] ou [3].

Seja $\phi$ dada por $\phi(x)=\frac{\|x\|^{2}}{2}$, onde $x \in X$. Como esta função é convexa e contínua, uma aplicação do Teorema de Hahn-Banach mostra que $\phi$ é subdiferenciável em qualquer ponto. De fato, como já vimos temos

$$
\partial \phi(x)=J(x)=\left\{x^{*} \in X^{*} ;\left\langle x^{*}, x\right\rangle=\left\|x^{*}\right\|\|x\| \text { e }\left\|x^{*}\right\|=\|x\|\right\} .
$$

$J$ é a chamada aplicação de dualidade.

Usando $J$ definimos:

$$
\begin{aligned}
& (x, y)_{+}=\sup \left\{\left\langle x^{*}, y\right\rangle ; x^{*} \in J(x)\right\} \\
& (x, y)_{-}=\inf \left\{\left\langle x^{*}, y\right\rangle ; x^{*} \in J(x)\right\}
\end{aligned}
$$

chamados produtos semi-internos.

Definição 1.37 Um operador $A: X \rightarrow 2^{X}$ é dito acretivo se para todo $\lambda \geq 0, x^{\prime} \in A x \in$ $y^{\prime} \in$ Ay vale a desigualdade

$$
\|x-y\| \leq\left\|(x-y)+\lambda\left(x^{\prime}-y^{\prime}\right)\right\|
$$

Denotaremos por $D(A)$ e $R(A)$ o domínio e a imagem de $A$, respectivamente, dados por:

$$
\begin{gathered}
D(A)=\{x \in X ; A x \neq \emptyset\} \\
R(A)=\bigcup_{x \in D(A)} A x .
\end{gathered}
$$

O conceito de operador acretivo foi primeiramente introduzido por T. Kato [32] e por F. E. Browder [11]. É uma extensão natural do conceito de operador monótono num espaço de Hilbert.

É comum identificar o operador $A$ com o seu gráfico em $X \times X$, isto é,

$$
\{(x, y) \in X \times X ; y \in A x \text { e } x \in D(A))\} .
$$

Assim, se $x^{\prime} \in A x$, escrevemos $\left(x, x^{\prime}\right) \in A$.

Denotamos por $A^{-1}$ o operador dado por $x \in A^{-1} y$ se, e somente se, $y \in A x$. O conjunto de operadores é ordenado pela inclusão, isto é, $A \subseteq B$ se, $D(A) \subseteq D(B)$ e se $A x \subseteq B x$, para todo $x \in D(A)$. Dados operadores $A$ e $B, \lambda$ e $\mu$ em $\mathbf{R}$, definimos $(\lambda A+\mu B)(x)=$ $\{\lambda y+\mu z ; y \in A x$ e $z \in B x\}$, claramente $D(\lambda A+\mu B)=D(A) \cap D(B)$.

Para um operador acretivo $A$ e $\lambda>0$ definimos:

$$
\begin{aligned}
& J_{\lambda}=(I+\lambda A)^{-1}, \\
& A_{\lambda}=\frac{1}{\lambda}\left(I-J_{\lambda}\right),
\end{aligned}
$$

chamados, respectivamente, de o resolvente e a aproximação de Yosida de $A$.

Observe que $D\left(J_{\lambda}\right)=R(I+\lambda A)=D\left(A_{\lambda}\right)$ e $A$ é acretivo se e, somente se, $A_{\lambda}$ é contração. 
Lema 1.38 Se A é um operador acretivo num espaço de Banach $X$, entẫo:

1) $A_{\lambda}$ é unívoca, Lipschitziana com constante $2 / \lambda$ e também acretiva para todo $\lambda>0$.

2) $A_{\lambda} \subset A J_{\lambda}$, para todo $\lambda>0$.

3) $A_{\lambda+\mu}=\left(A_{\lambda}\right)_{\mu}$

4) $J_{\lambda}$ é não-expansiva.

5) $J_{\lambda} x \in J_{\mu}\left(\frac{\mu}{\lambda} x+\frac{\lambda-\mu}{\lambda} J_{\lambda} x\right), \forall x \in D\left(J_{\lambda}\right), \lambda>0$ e $\mu \geq 0$

Para a prova consulte a referência [3] ou [37].

Definição 1.39 Seja $A$ um operador acretivo em $X$. Dizemos que $A$ é maximal se toda extensão de $A$ coincide com $A$ e dizemos que $A$ é $m$-acretivo se

$$
R(I+\lambda A)=X
$$

para todo $\lambda \geq 0$

Kato [32] provou que se $R(I+\lambda A)=X$ para algum $\lambda>0$, então $A$ é $m$-acretivo. Num espaço de Banach todo operador $m$-acretivo é maximal. A recíproca é falso; é verdadeira quando o espaço $X$ for, por exemplo, Hilbert. Veja [3] para este e outros resultados.

Lema 1.40 Seja A um operador m-acretivo. Então:

1) A é fechado.

2) Se $\left(x_{\lambda}\right)$ converge para $x$ e $A_{\lambda} x_{\lambda}$ converge para y quando $\lambda$ tende a zero, então $y \in A x$.

Em geral se $R(I+A)$ é fechado, então $A$ é fechado. Veja [59] ou [3] para outros resultados.

Lema 1.41 1) Se $\lambda$ é um número real satisfazendo

$$
[x, y]_{-} \leq \lambda \leq[x, y]_{+}
$$

entâo, existe $x^{*} \in J(x)$ tal que $\lambda\|x\|=\left\langle x^{*}, y\right\rangle$. Em particular,

$$
\begin{aligned}
& (x, y)_{+}=\|x\|[x, y]_{+}, \\
& (x, y)_{-}=\|x\|[x, y]_{-} .
\end{aligned}
$$

2) Sejam $x:[0, T] \rightarrow X$ e $m(t)=\|x(t)\|^{2}$. Se $x_{+}^{\prime}$ e $x_{-}^{\prime}$ existem para algum $t \in(0, T)$, então

$$
\begin{aligned}
& m_{+}^{\prime}=2\left(x(t), x_{+}^{\prime}(t)\right)_{+}, \\
& m_{-}^{\prime}=2\left(x(t), x_{-}^{\prime}(t)\right)_{-} .
\end{aligned}
$$

3) $(x, y)_{+}$éscs e $(x, y)_{-}$é sci.

Veja as demonstrações em [37].

Uma notação também usual para o produto $(x, y)_{+}$é $\langle y, x\rangle_{s}$, a qual foi primeiramente introduzido por Crandall-Liggett e por Sato.

A aplicação de dualidade $J$ está relacionada à regularidade da norma do espaço. 
Definição 1.42 Um espaço normado $X$ é chamado estritamente convexo se a bola unitária $S$ de $X$ é estritamente convexa, isto é, a fronteira de $S$ não contém segmentos de reta.

Definição 1.43 Um espaço normado $X$ é uniformemente convexo se para cada $\varepsilon \in(0,2]$ existe um $\delta(\varepsilon)=\delta>0$ tal que $\|x\| \leq 1,\|y\| \leq 1$ e $\|x-y\| \geq \varepsilon$ implicam $\|x+y\| \leq 2(1-\delta)$.

Lembramos que todo espaço de Banach uniformemente convexo é reflexivo. Asplund provou que todo Banach reflexivo $X$ pode ser renormado de modo que $X$ e $X^{*}$ sejam estritamente convexos. Mais sobre o assunto pode ser encontrado em [30].

Teorema 1.44 Seja X um espaço de Banach

1) Se $X^{*}$ é estritamente convexo, então a aplicação de dualidade $J$ é unívoca e contínua de $X$ em $X_{\omega^{*}}^{*}$.

2) Se $X^{*}$ é uniformemente convexo, então $J$ é uniformemente contínua em todo subconjunto limitado de $X$.

Demonstração em [30] ou [3].

Uma caracterização para operador acretivo é a seguinte:

$$
\left[x-x^{\prime}, y-y^{\prime}\right]_{+} \geq 0
$$

para cada $x, x^{\prime} \in D(A), y \in A x$ e $y^{\prime} \in A x^{\prime}$.

Claramente $A$ é acretivo se, se e somente se, $\left(x-x^{\prime}, y-y^{\prime}\right)_{+} \geq 0$.

Kato [32] provou que a acretividade de um operador $A$ é equivalente a

$$
\left\langle y-y^{\prime}, \omega\right\rangle \leq 0
$$

para todo $y \in A x$ e $y^{\prime} \in A x^{\prime}$ e algum $\omega \in J\left(x-x^{\prime}\right)$.

As propriedades dos operadores acretivos dependem muito mais das propriedades geométricas do espaço em que estão definidos do que os operadores monótonos.

Teorema 1.45 Seja $X$ um espaço de Banach.

1) $X$ é Gateaux suave, se somente se, $A(u)$ é convexo para todo $A: X \rightarrow 2^{X}$ acretivo maximal e para todo $u \in D(A)$.

2) A dimensấo do espaço gerado por $\{J(u)\}$ é finita se, e somente se, $A(u)$ é w-fechado para qualquer operador acretivo maximal $A$ e qualquer $u \in D(A)$.

Logo, para um espaço $X$ Gateaux suave, $A(u)$ é convexo e fraco-fechado e portanto, $A(u)$ é convexo fechado para todo operador $A$ acretivo maximal em $X$ e para todo $u \in D(A)$. Caracterizar os espaços de Banach $X$ tais que todo operador acretivo maximal $A: X \rightarrow 2^{X}$ tem $A(u)$ convexo ou $A(u) w$-fechado é um problema ainda em aberto. Veja [59].

Usaremos também as noções de continuidade e de seleção para aplicações do tipo $A$ : $X \rightarrow 2^{Y}$.

Definição 1.46 Sejam $X$ e $Y$ espaços topológicos e $F: X \rightarrow 2^{Y}$ uma aplicação tal que $F(x) \neq \emptyset, \forall x \in X$. Dizemos que $F$ é semicontínua superior (scs) em $x_{0} \in X$ se para qualquer vizinhança $V\left(F\left(x_{0}\right)\right)$ existir uma vizinhança $V\left(x_{0}\right)$ tal que

$$
F\left(V\left(x_{0}\right)\right) \subset V\left(F\left(x_{0}\right)\right) .
$$


A aplicação $F$ é semicontínua inferior (sci) em $x_{0} \in X$ se para qualquer $y \in F\left(x_{0}\right)$ e qualquer vizinhança $V(y)$ existir uma vizinhança $N\left(x_{0}\right)$ tal que se $x \in N\left(x_{0}\right)$, então

$$
F(x) \cap V(y) \neq \emptyset \text {. }
$$

Se $F$ for scs e sci em $x_{0}$ dizemos que $F$ é contínua em $x_{0}$.

Definição 1.47 Uma seleção para $F: X \rightarrow 2^{Y}$ é uma função $f: X \rightarrow Y$ tal que $f(x) \in$ $F(x), \forall x \in X$.

A existência de seleções contínuas tem aplicações, por exemplo, na Análise Convexa onde estuda-se a existência de seleções contínuas para o subdiferencial de uma função convexa.

Se $A: X \rightarrow 2^{X}$ é um operador acretivo, é fácil ver que toda seleção de $A$ é também acretiva. Além disso, se $A$ admite uma seleção $m$-acretiva, então $A$ é um operador $m$-acretivo.

Existe ainda outra noção de acretividade, que a título de curiosidade incluiremos aqui.

Definição $1.48 U m$ operador $A$ é $k$-acretivo, para algum $k \geq 0$, se para todo $\lambda \geq 0$,

$$
\left\|\left(x-x^{\prime}\right)-\lambda k\left(y-y^{\prime}\right)\right\| \leq\left\|\left(x-x^{\prime}\right)+\lambda\left(y-y^{\prime}\right)\right\|,
$$

onde $x, x^{\prime} \in D(A), y \in A x$ e $y^{\prime} \in A x^{\prime}$.

Quando $k=0$ obtemos a noção anterior de operador acretivo. Além disso, todo operador $k$-acretivo é também $k^{\prime}$-acretivo sempre que $0 \leq k^{\prime} \leq k$. Os operadores $k$-acretivos estão associados a aplicações assintoticamente regulares. Tais aplicações são importantes em problemas de programação convexa e princípios variacionais. Sabemos que muitos destes problemas podem ser convertidos em encontrar uma solução para o problema de inclusão

$$
A x \ni 0
$$

onde $A$ é um operador monótono maximal sobre um espaço de Hilbert $H$. O algoritmo do ponto próximo é um método eficiente para resolver tais inclusões. Este método se baseia no fato que a aplicação $P=(I+A)^{-1}$ é unívoca e não expansiva sobre todo o Hilbert $H$. Este algoritmo gera, para qualquer ponto inicial $x_{1}$, uma sequência de iteradas $\left(x_{n}\right)$ satisfazendo

$$
\left\|x_{n+1}-P x_{n}\right\| \leq \varepsilon_{n}
$$

$\operatorname{com}\left(\varepsilon_{n}\right)$ reais positivos tais que $\sum \varepsilon_{n}<\infty$. A questão é se a sequência $\left(x_{n}\right)$ converge para um ponto fixo de $P$, se tal ponto existe. Temos o seguinte resultado:

Teorema 1.49 [Khang] Admitindo $P: C \rightarrow C$ estritamente não expansiva com pelo menos um ponto fixo e $C$ convexo limitado de um espaço de Banach $X$, se $Q^{-1}=(I-P)^{-1}$ é semicontínua superior na origem, então o algoritmo do ponto próximo aplicado a $P$ converge a um ponto fixo de $P$. 
Um operador $k$-acretivo $A$ é dito $m-k$-acretivo se $R(I+\lambda A)=X$. Para um operador $A$ $m-k$-acretivo com $k>0, J_{\lambda}$ é estritamente $t=\frac{k}{1-k}$-não expansivo. Seja $P=t I+(1-t) J_{\lambda}$ e suponha $A^{-1}(0) \neq \emptyset$. Se $A^{-1}$ é semicontínua superior na origem, entâo $Q^{-1}=(I-P)^{-1}$ também o é. Logo, o algoritmo do ponto próximo aplicado a $P$ gera uma sequência que converge para um ponto fixo $x$ de $P$, ou seja,

\section{$A x \ni 0$.}

Mais sobre regularidade assintótica poder ser encontrado em [33].

Definição 1.50 Sejam $X$ e $Y$ espaços de Banach. Um operador $T: X \rightarrow Y$ (nâo necessariamente linear) é chamado compacto se ele é contínuo e leva subconjuntos limitados em subconjuntos relativamente compactos.

O seguinte resultado também será útil e a prova pode ser encontrada em [29] ou [28].

Teorema 1.51 Seja $T_{\lambda}: D \subset X \rightarrow X$ uma familia de operadores compactos. Se $\lim _{\lambda} T_{\lambda}=$ $T$ uniformemente sobre subconjuntos limitados de $D$, entâo $T$ é compacto.

Também precisaremos dos seguintes resultados devido a D. B. Khang [33].

Teorema 1.52 (D. B. Khang) Sejam $X$ um espaço de Banach, $\left(v_{n}\right)$ e $\left(w_{n}\right)$ sequências em $X$. Suponha que para uma constante $0<t<1$ as seguintes afirmaçôes sejam satisfeitas:

1) $\left(\left\|v_{n}\right\|\right)$ é não-crescente.

2) $\left\|w_{n}\right\| \leq\left\|v_{n}\right\|, \forall n e\left(\left\|\sum_{1}^{N} w_{n}\right\|\right)_{N}$ é limitado.

3) $v_{n+1}=(1-t) v_{n}+t w_{n}, \forall n$.

Então, para todo $\varepsilon>0$ existe um inteiro $N$ tal que

$$
\left\|v_{n}\right\| \leq \varepsilon
$$

sempre que $n \geq N$.

Além disso, $N$ pode ser determinado como se segue: se $s=1-t e$

$$
M=\sup \left\{\left\|\sum_{i=1}^{n+m} w_{i}\right\| ; n \text { e } m \text { são naturais }\right\}<\infty,
$$

escolha $k \geq \max \left\{\frac{1}{t}, \frac{2 M}{\varepsilon}\right\}$ e um número positivo $r<\frac{\varepsilon s^{k}}{2}$. Então,

$$
N=k\left\{\left[\frac{\left\|v_{0}\right\|-\varepsilon}{r}\right]+1\right\}
$$

onde $[\alpha]$ é o maior inteiro menor ou igual a $\alpha$.

Teorema 1.53 [Khang] Seja $D$ subconjunto convexo e limitado de um espaço normado $X$ e $T: D \rightarrow C B(D)$ uma aplicação não expansiva. Para uma sequência $\left(t_{n}\right)$ de reais satisfazendo $0<t_{n} \leq t<1$ e $\sum t_{n}=\infty$, seja $P_{n}=\left(1-t_{n}\right) I+t_{n} T$. Então $\left(P_{n}\right)$ é assintoticamente regular. 


\subsection{Semigrupos e operadores de evolução}

Definição 1.54 Sejam $D \subset X$ e $X$ um espaço de Banach. Uma família de operadores

$$
S=\{S(t) ; S(t): D \rightarrow D, t \geq 0\},
$$

é chamada um $C^{0}$ semigrupo em $D$ se:

1) $S(t+s)=S(t) S(s)$ para cada, $t, s \geq 0$

2) $S(0)=I$,

3) $\lim _{t \searrow 0} S(t) x=x$, para cada $x \in D$.

Se além das condições acima a família $S$ satisfaz

4) $\|S(t) x-S(t) y\| \leq\|x-y\|, x$ e $y \in D$ e $t \geq 0$, dizemos que $S$ é um semigrupo não expansivo.

A fórmula exponencial de Crandall-Liggett para um operador $m$-acretivo define um semigrupo de aplicações não-expansivas, i.e,

$$
S_{A}(t) x=\lim _{n \rightarrow \infty}\left(I+\frac{t}{n} A\right)^{n} x
$$

onde $x \in \overline{D(A)}$ e $t \geq 0$, tem as propriedades acima. Veja [3] ou [47].

Este semigrupo é dito gerado por $-A$. Se cada operador $S(t), t>0$ é compacto, dizemos que o semigrupo é compacto.

Definição 1.55 Sejam X um espaço de Banach,

$$
D=\{D(t) \subset X ; 0 \leq s \leq t \leq T\}
$$

uma família de subconjuntos não vazios, onde $[0, T] \subset \mathbf{R}$. Uma família de operadores

$$
U=\{U(t, s): D(s) \rightarrow D(t), 0 \leq s \leq t \leq T\},
$$

é chamado um operador de evolução com relação a $D$ se satisfaz:

1) Para cada $0 \leq s \leq T, U(s, s)=I$ em $D(s)$.

2) $U(t, s) U(s, r)=U(t, r), \forall 0 \leq r \leq s \leq t \leq T$.

3) $t \rightarrow U(t, s) x$ é contínua em $[s, T]$, para cada $x \in D(s)$ e $s \in[0, T]$.

Veja [47] para outros resultados.

\subsection{Medidas de não compacidade}

Comecemos com a medida de não compacidade $\alpha$ de Kuratowski [37].

Definição 1.56 Seja $B$ um subconjunto limitado de um espaço de Banach $X$, definimos $\alpha(B)=\inf \{d>0 ; B$ é coberto por um número finito de conjuntos com diâmetro máximo $d\}$. 
Note que $\alpha(B) \leq \operatorname{diam}(B)$, onde $\operatorname{diam}(B)$ denota o diâmetro de $B$.

Proposição 1.57 Sejam $A$ e $B$ subconjuntos limitados de $X$.

1) $\alpha(A)=0$ se, e somente se, $\bar{A}$ é compacto.

2) $\alpha(A)=\alpha(\bar{A})$.

3) $\alpha(A \cup B)=\max \{\alpha(A), \alpha(B)\}$.

Quando consideramos subconjuntos de $C([0, T] ; X)$ é conveniente usar a seguinte notação: se $K \subset C([0, T] ; X), K(t)$ e $K([0, T])$ representam os conjuntos dados por $\{f(t) ; f \in K\}$ e $\bigcup\{f(t) ; f \in K\}$ respectivamente.

$t \in[0, T]$

O próximo resultado está relacionado com os teoremas 1.30 e 1.31 .

Proposição 1.58 Se $K \subset C([0, T] ; X)$ é qualquer conjunto limitado e equicontínuo, então

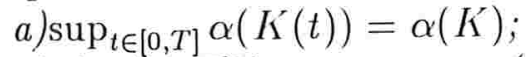

b) $\alpha(K([0, T]))=\sup _{t \in[0, T]} \alpha(K(t))$.

Para um conjunto $B \subset X$ limitado, a medida $\gamma$ de não compacidade de Hausdorff é definida por:

Definição $1.59 \gamma(B)=\inf \{r>0: B$ é coberto por um número finito de bolas de raio $r\}$.

A medida de não compacidade fraca (de DeBlase [17]) é dada por:

Definição $1.60 \beta(B)=\inf \{t>0$ : existe um compacto fraco $C$ tal que $B \subset C+t \bar{S}\}$, onde $\bar{S}$ denota a bola unitária fechada de $X$.

\section{PROPRIEDADES:}

Sejam $A$ e $B$ subconjuntos limitados de $X$.Então:

1) $\beta(A)=\beta\left(\bar{A}^{w}\right)$.

2) $A \subset B$ implica $\beta(A) \leq \beta(B)$.

3) $\beta(A)=0$ se, e somente se, $\bar{A}^{w}$ é fraco compacto.

4) $\beta(A)=\beta(\operatorname{co}(A))$.

5) $\beta(A+B) \leq \beta(A)+\beta(B)$.

6) $\beta(A) \leq \operatorname{diam}(A)$ e $\beta(A) \leq \alpha(A)$.

Veja DeBlase [17] ou Kuratowski [37] para as demonstrações. Veja também [27].

Dizemos que $g \in C\left(\mathbf{R}^{+}, \mathbf{R}^{+}\right)$é uma função de Kamke [2] se $u(t) \equiv 0$ é a única solução de

$$
\left\{\begin{array}{l}
u^{\prime}=g(u) \\
u(0)=0
\end{array}\right.
$$

em $[0, T]$.

No Capítulo 3 vamos usar funções de Kamke em associação com medida de não compacidade fraca em hipóteses que nos garantam existência de solução de equações diferenciais em espaços de Banach.

Definição 1.61 Dizemos que $S: X \rightarrow X$ satisfaz à condição de Kamke associada à função de Kamke $g$ se

$$
\beta(S(N)) \leq g(\beta(N)), \forall N \subset X \text { limitado. }
$$

Veja também [14] para mais informações e aplicações desta condição. 


\section{Capítulo 2}

\section{Soluções locais de problemas abstratos de Cauchy}

\subsection{Introdução}

Neste capítulo, vamos estudar problemas abstratos de Cauchy associados a uma família $A(t), 0 \leq t \leq T$, de operadores $m$-acretivos. Mais precisamente, vamos abordar problemas do tipo $P A(t) C(F u ; x)$, isto é,

$$
\left\{\begin{array}{l}
\frac{d u}{d t}+A(t) u(t) \ni(F u)(t), 0 \leq t \leq T \\
u(s)=x \in \overline{D(A(s))}, 0 \leq s \leq t \leq T
\end{array}\right.
$$

onde $F: C([0, T] ; X) \rightarrow C([0, T] ; X)$ é um operador contínuo e $D(A(s))$ é o domínio do operador $A(s)$.

Estaremos sempre supondo que $F$ seja um operador causal, isto é, se as imagens $F u$ e $F v$ são iguais em $[0, a] \subset[0, T]$, então $u$ e $v$ são iguais em $[0, a]$. Duas outras hipóteses serão usadas em todo o resto deste texto, abaixo designadas por $\mathbf{H 1}$ e $\mathbf{H} 2$.

Seja $A(t), 0 \leq t \leq T$, uma família de operadores $m$-acretivos sobre um espaço de Banach $X$. Consideremos as seguintes condições:

H1- Para $a<0 \leq s \leq t \leq T<b, \lambda \geq 0$, podendo acontecer $a=-\infty$ ou $b=\infty$, existem $h:(a, b) \rightarrow X$ contínua e $\bar{L}: \mathbf{R}^{+} \rightarrow \mathbf{R}^{+}$limitada sobre subconjuntos limitados satisfazendo

$$
\left\|x_{1}-x_{2}\right\| \leq\left\|\left(x_{1}-x_{2}\right)+\lambda\left(y_{1}-y_{2}\right)\right\|+\|h(t)-h(s)\| L\left(\left\|x_{2}\right\|\right)
$$

para todo $\left(x_{1}, y_{1}\right) \in A(t),\left(x_{2}, y_{2}\right) \in A(s)$.

H2- Se $t_{n} \downarrow t$ em $(s, T], x_{n} \in D\left(A\left(t_{n}\right)\right)$ e $x_{n} \rightarrow x$ em $X$, então $x \in \overline{D(A(t))}$.

Observamos que em $\mathbf{H 1}, s=t$ e $h \equiv 0$ ou $L \equiv 0$ obtemos o conceito de acretividade.

A existência e unicidade de soluções de problemas abstratos do tipo $P A(t) C(f ; x)$, com $f \in L^{1}(0, T ; X)$ e $A(t)$ satisfazendo hipóteses $\mathbf{H} 1$ e $\mathbf{H} 2$ foi obtida por meio de esquemas 
discretos (DS). Quando $F \equiv 0$ e $A(t)$ é uma família de operadores $m$-acretivos, obtemos um operador de evolução $U$ que dá a solução do problema,

$$
U=\{U(t, s): \overline{D(A(s))} \rightarrow \overline{D(A(t))}, 0 \leq s \leq t \leq T\}
$$

dado por:

$$
U(t, s) x=\lim _{n \rightarrow \infty} \prod_{k=1}^{n}\left(I+\frac{t-s}{n} A\left(s+k \frac{t-s}{n}\right)\right)^{-1} x
$$

Veja Pavel [47] para uma prova destas afirmações.

Pode-se provar que se a família $A(t), 0 \leq t \leq T$, for $m$-acretiva e valem $\mathbf{H} \mathbf{1}$ e $\mathbf{H 2}$, entào os domínios $\overline{D(A(t))}$ independem de $t$. Veja [47].

Logo, podemos supor sempre que

$$
\overline{D(A(t))}=\overline{D(A(0))}:=D
$$

e $u(0)=x \in D$.

Em geral, $D(A(t)) \subset \hat{D}(A(t)) \subset \overline{D(A(t))}$, onde

$$
\hat{D}(A(t))=\left\{x \in \overline{D(A(t))}: \liminf _{h \searrow 0} \frac{1}{h}\|U(t+h, t) x-x\|<\infty\right\},
$$

é denominado o domínio generalizado de $A(t)$. Este conjunto desempenha um papel importante na teoria das equações de evolução em espaços de Banach, pois sob algumas condições e $x \in \hat{D}(A(s))$, a aplicação

$$
t \mapsto U(t, s) x
$$

é Lipschitz contínua em [ $s, T]$. Veja [47].

Antes de enunciarmos o principal resultado desta secção precisamos introduzir alguns conceitos.

Definição 2.1 Se $x, y \in X$, definimos

$$
\begin{gathered}
(x, y)_{+}=\langle y, x\rangle_{s}=\lim _{h \downarrow 0} \frac{\|x+h y\|^{2}-\|x\|^{2}}{2 h} \\
{[x, y]_{+}=\langle y, x\rangle_{+}=\lim _{h \downarrow 0} \frac{\|x+h y\|-\|x\|}{h}} \\
(x, y)_{-}=\langle y, x\rangle_{i}=\lim _{h \uparrow 0} \frac{\|x+h y\|^{2}-\|x\|^{2}}{2 h} \\
{[x, y]_{-}=\langle y, x\rangle_{-}=\lim _{h \uparrow 0} \frac{\|x+h y\|-\|x\|}{h}}
\end{gathered}
$$

O seguinte conceito de solução integral em $[s, T]$ para problemas abstratos $P A(t) C\left(f: x_{0}\right)$, é devido a Pavel [47]. 
Definição 2.2 Por uma soluçâo integral em $[s, T]$ para problemas abstratos de Cauchy $P A(t) C\left(f ; x_{0}\right), x_{0} \in \overline{D(A(s))}$, sob a condiçấo $\mathbf{H 1}$, entendemos uma função contínua $u:[s, T] \rightarrow X$ satisfazendo:

1) $u(s)=x_{0}$

2) $u(t) \in \overline{D(A(t))}$ para quase todo $t \in[s, T]$,

3) $\|u(\bar{t})-x\|^{2} \leq\|u(t)-x\|^{2}+2 \int_{t}^{\bar{t}}\left[\langle f(\bar{s})-y, u(\bar{s})-x\rangle_{s}+C\|u(\bar{s})-x\|\|h(\bar{s})-h(r)\|\right] d \bar{s}$, onde $C=\max \left\{L\left(c_{1}\right), L(\|x\|)\right\}$ e $c_{1}=\sup \{\|u(t)\| ; s \leq t \leq T\}, s \leq t \leq \bar{s} \leq \bar{t} \leq T, r \in[s, T]$ $e(x, y) \in A(r)$.

Definição 2.3 Uma solução forte de PA(t)C(f; $\left.x_{0}\right)$ em $[s, T]$ é uma função $u:[s, T] \rightarrow X$ satisfazendo:

1) u é contínua em $[s, T]$ e absolutamente continua em subconjuntos compactos de $(s, T)$.

2) $u(t) \in D(A(t))$, para quase todo $t \in(s, T)$.

3) $u$ é fortemente diferenciável em quase todo $t \in(s, T)$ e satisfaz ao $P A(t) C\left(f ; x_{0}\right)$ quase sempre em $(s, T)$.

Teorema 2.4 (Pavel) Sejam $A(t), 0 \leq t \leq T$, uma familia de operadores $m$-acretivos que satisfaz $\mathbf{H 1}$ e $\mathbf{H 2}$, e $f:[0, T] \rightarrow X$ uma função de $L^{1}([0, T] ; X)$. Então, existe uma única soluçâo integral de

$$
\left\{\begin{array}{l}
\frac{d u}{d t}+A(t) u(t) \ni f(t), 0 \leq t \leq T \\
u(s)=x \in \overline{D(A(s))}, 0 \leq s \leq t \leq T
\end{array}\right.
$$

Se $u$ e $v$ são soluções integrais dos problemas $P A(t) C\left(f ; x_{1}\right)$ e $P A(t) C\left(g ; x_{2}\right)$, respectivamente, com $x_{i} \in \overline{D(A(s))}, A(t), 0 \leq t \leq T$, é uma família de operadores $m$-acretivos satisfazendo $\mathbf{H 1}$ e $\mathbf{H} 2$, e $f, g:[0, T] \rightarrow \bar{X}$ são funções de $C([0, T] ; X)$, então tem-se a seguinte desigualdade:

$$
\|u(t)-v(t)\| \leq\|u(\tau)-v(\tau)\|+\int_{\tau}^{t}\|f(r)-g(r)\| d r,
$$

$0 \leq s \leq \tau \leq t \leq T$

Definição 2.5 Denotaremos por $B_{x_{0}}$ o operador, chamado de Bénilan-Pavel, que a cada $f \in L^{1}(0, T ; X)$ associa a única solução integral de $P A(t) C\left(f ; x_{0}\right)$ em $[0, T]$.

A desigualdade acima mostra que o operador de Bénilan-Pavel depende continuamente dos dados iniciais.

Para $a \in(0, T]$, definimos os operadores $E_{a}$ e $T_{a}$ que estendem e truncam, respectivamente, funções contínuas:

$$
\begin{aligned}
& E_{a}: C([0, a] ; X) \rightarrow C([0, T] ; X) \\
& T_{a}: C([0, T] ; X) \rightarrow C([0, a] ; X)
\end{aligned}
$$

dados por

$$
\left(E_{a} u\right)(t)= \begin{cases}u(t), & \text { se } t \in[0, a] \\ u(a), & \text { se } t \in[a, T]\end{cases}
$$




$$
\left(T_{a} u\right)(t)=u(t) .
$$

Claramente, $T_{a}$ e $E_{a}$ são contínuos. Usamos estes operadores para definir solução integral local para problemas do tipo

$$
\left\{\begin{array}{l}
\frac{d u}{d t}+A(t) u(t) \ni(F u)(t), 0 \leq t \leq T \\
u(s)=x \in \overline{D(A(s))}, 0 \leq s \leq t \leq T
\end{array}\right.
$$

Definição 2.6 Uma soluçâo (integral) local em $[0, r], 0<r \leq T$, para o problema $P A(t) C(F u ; x)$, é uma funçâo contínua $u:[0, r] \rightarrow D$, com $u(0)=x$ que é ponto fixo de

$$
T_{r} B_{x} F E_{r}: C([0, r] ; X) \rightarrow C([0, r] ; X) .
$$

Uma soluçấo local em $[0, s)$ é uma solução local em $[0, r]$, para todo $0 \leq r<s$.

A seguinte hipótese será usada em todo o restante deste trabalho.

H3- Seja $N$ um conjunto de operadores contínuos (causais) de $C([0, T] ; X)$ em $C([0, T] ; X)$. Suponha que $N$ leva subconjuntos limitados em subconjuntos uniformemente integráveis. isto é,

$$
N(B)=\bigcup_{F \in N} F(B)
$$

é uniformemente integrável para cada $B$ limitado.

Como estamos supondo que $F$ seja causal, toda solução local em $[0, r] \subset[0, T]$ é também solução local em $[0, s]$ com $0 \leq s \leq r$. Agora estamos prontos para demonstrar o principal resultado deste capítulo.

\subsection{Teorema de existência}

Teorema 2.7 (Teorema de Existência): Sejam $A(t), 0 \leq t \leq T$, uma família de operadores m-acretivos em um espaço de Banach $X$ que satisfaz $\mathbf{H 1}$ e $\mathbf{H} 2$, e $N=\{F\}$ como em H3. Suponha que $x \in D=\overline{D(A(0))}$ e que $F$ satisfaz a uma das seguintes condições:

H4- $B_{x} F$ é compacto,

H5-Existe $\varphi: C([0, T] ; X) \rightarrow \mathbf{R}^{+}$sci tal que

$$
\left\|v-B_{x} F v\right\|_{\infty} \leq \varphi(v)-\varphi\left(B_{x} F v\right),
$$

para todo $v \in C([0, T] ; X)$,

H6-Existe $\varphi: \mathbf{R}^{+} \rightarrow \mathbf{R}^{+}$scs tal que $\varphi(t)<t e$

$$
\left\|B_{x} F u-B_{x} F v\right\|_{\infty} \leq \varphi(\|u-v\|),
$$

para todos $u$ e $v \in C([0, T] ; X)$,

Então, existe $\delta>0$ tal que o problema $P A(t) C(F u ; x)$ tem pelo menos uma solução integral no intervalo $[0, \delta]$. Além disso, o conjunto de soluções $I(F)$ é limitado e fechado em $C([0, \delta] ; X)$. 
Prova: Seja $u_{0}$ a única solução integral de $P A(t) C(0 ; x)$ (que existe e está definida em $[0, T]$, pelo Teorema 2.4). Para $\varepsilon>0$, seja

$$
C_{\varepsilon}=\left\{u \in C([0, T] ; X):\left\|u(t)-u_{0}(t)\right\| \leq \varepsilon, t \in[0, T] \text { e } u(0)=x\right\} .
$$

Este conjunto é não vazio, convexo, limitado e fechado em $C([0, T] ; X)$. Para $r \in(0, T]$, escrevemos

$$
C_{\varepsilon}^{r}=T_{r}\left(C_{\varepsilon}\right)
$$

que também é convexo, limitado e fechado.

Consideremos o conjunto

$$
S_{\varepsilon}=\left\{u \in C([0, T] ; X) ;\|u\|_{\infty} \leq\left\|u_{0}\right\|_{\infty}+\varepsilon\right\} .
$$

Então, claramente $E_{r}\left(C_{\varepsilon}^{r}\right) \subset S_{\varepsilon}$. Como $S_{\varepsilon}$ é limitado, segue da hipótese H3 que $F\left(S_{\varepsilon}\right)$ é uniformemente integrável e assim para o $\varepsilon>0$ dado existe $\delta \in(0, T]$ tal que

$$
\int_{0}^{\delta}\|f(s)\| d s \leq \varepsilon, \forall f \in F\left(S_{\varepsilon}\right)
$$

Tomando $r=\delta$ e $v \in C_{\varepsilon}^{\delta}$, temos

$$
\begin{aligned}
\left\|\left(T_{\delta} B_{x} F E_{\delta} v\right)(s)-T_{\delta} u_{0}(s)\right\| & =\left\|\left(B_{x} F E_{\delta} v\right)(s)-u_{0}(s)\right\| \\
& \leq \int_{0}^{\delta}\left\|F E_{\delta} v(s)\right\| d s \leq \varepsilon,
\end{aligned}
$$

pois $\left(B_{x} F E_{\delta} v\right)$ é solução integral de $P A(t) C\left(F E_{\delta} v ; x\right)$ e $\left(F E_{\delta} v\right) \in C([0, T] ; X)$. Logo, $\left(T_{\delta} B_{x} F E_{\delta}\right)\left(C_{\varepsilon}^{\delta}\right) \subset C_{\varepsilon}^{\delta}$.

Segue das hipóteses $\mathbf{H 4}$ ou $\mathbf{H 5}$ ou $\mathbf{H 6}$ e dos teoremas de ponto fixo do Capítulo 1 que existe uma solução local em $[0, \delta]$ para $P A(t) C(F u ; x)$.

É fácil ver que o conjunto de soluções $I(F)$ é limitado. Vamos verificar que é fechado. Note que $\mathbf{H} 6$ implica que $I(F)$ é unitário, nos demais casos nada podemos garantir. Vamos admitir que todos os elementos de $I(F)$ estejam definidos em $[0, \delta]$. Seja $\left(u_{n}\right)$ uma sequência de elementos de $I(F)$ convergente para $u$. Provaremos que $u$ é ponto fixo de $\left(T_{\delta} B_{x} F E_{\delta}\right)$, em $[0, \delta]$. Dado $\varepsilon_{1}>0$ existe um natural $n_{0}$ tal que

$$
\left\|u_{n}-u\right\|_{\infty}<\varepsilon_{1}, \forall n \geq n_{0} .
$$

Como $B_{x} F$ é contínuo, para este $\varepsilon_{1}>0$ dado existe $n_{1} \geq n_{0}$, tal que

$$
\left\|B_{x} F u_{n}-B_{x} F u\right\|_{\infty}=\left\|u_{n}-B_{x} F u\right\|_{\infty}<\varepsilon_{1},
$$

para todo $n \geq n_{1}$.

Tomando o limite quando $n$ tende ao infinito, obtemos

$$
\left\|u-B_{x} F u\right\|_{\infty} \leq \varepsilon_{1} .
$$

Logo, $B_{x} F u=u$. Concluímos assim a prova do teorema. 
Exemplo 2.8 Seja $F: C([0, T] ; X) \rightarrow C([0, T] ; X)$ contínuo tal que para cada $K \subset$ $C([0, T] ; X)$ limitado exista $g_{K} \in L^{1}\left(0, T ; \mathbf{R}^{+}\right)$tal que

$$
\|f(t)\| \leq g_{K}(t)
$$

para quase todo $t \in[0, T]$ e $f \in F(K)$. Então, se $\mathbf{H} 4$ ou $\mathbf{H 5}$ ou $\mathbf{H} 6$ é satisfeita, o problema $P A(t) C(F u ; x)$ tem solução local pelo teorema 2.7.

Exemplo 2.9 Seja $F: C([0, T] ; X) \rightarrow C([0, T] ; X)$ compacto. Como $F$ leva subconjuntos limitados em uniformemente integráveis e $B_{x}$ é contínuo então $B_{x} F$ é compacto. Pelo teorema 2.7, o problema PA(t)C(Fu;x)tem uma solução local.

Exemplo 2.10 Seja $F: X \rightarrow X$ uma contraçấo linear com constante $C<\frac{1}{T}$. Defina

$$
C([0, T] ; X) \ni u \mapsto \tilde{F}(u)=F(u(\cdot)) \in C([0, T] ; X) .
$$

Então, $\tilde{F}$ leva subconjuntos limitados em subconjuntos uniformemente integráveis e

$$
\begin{gathered}
\left\|B_{x} \tilde{F}(f)(t)-B_{x} \tilde{F}(g)(t)\right\| \leq \int_{0}^{t}\|\tilde{F}(f)(\tau)-\tilde{F}(g)(\tau)\| d \tau= \\
=\int_{0}^{t}\|F(f(\tau)-g(\tau))\| d \tau \leq C \int_{0}^{t}\|f(\tau)-g(\tau)\| d \tau \leq C t\|f-g\|_{\infty} .
\end{gathered}
$$

Logo,

$$
\left\|B_{x} \tilde{F} f-B_{x} \tilde{F} g\right\|_{\infty} \leq C T\|f-g\|_{\infty}
$$

e assim $B_{x} \tilde{F}$ satisfaz $\mathbf{H} 6 \operatorname{com} \varphi(t)=C T t$.

Exemplo 2.11 Se $B_{x} F$ é contração com constante $k<1$, então $B_{x} F$ satisfaz $\mathbf{H} 5$ com

$$
\varphi(v)=\frac{1}{1-k}\left\|v-B_{x} F v\right\|_{\infty}
$$

Veremos agora um exemplo simples da Teoria do Controle. Este exemplo sugere os resultados da próxima secção.

Exemplo 2.12 Consideremos o seguinte problema de controle:

$$
\inf _{u \in I(F)} \int_{0}^{T} V(u(t)) d t+R(u(T))
$$

sujeito ao seguinte problema abstrato:

$$
\left\{\begin{array}{l}
\frac{d u}{d t}+A(t) u(t) \ni(F u)(t), 0 \leq t \leq T \\
u(0)=0 \in \overline{D(A(0))}
\end{array}\right.
$$

onde $V, R: X \rightarrow \mathbf{R}^{+} \cup\{\infty\}$ são funçôes convexas, próprias e sci e $N=\{F\}$ satisfazendo H3. Suponha que exista $u_{F} \in I(F)$ tal que: 
- $V\left(u_{F}(\cdot)\right) \in L^{1}\left(0, T ; \mathbf{R}^{+}\right)$

- $R\left(u_{F}(T)\right)$ é finito.

Suponha que $\mathbf{H 4}$ ou $\mathbf{H 5}$ ou $\mathbf{H 6}$ seja satisfeita e que $I(F)$ seja w-compacto. Afirmamos que o problema de controle acima tem pelo menos um controle ótimo. Por simplicidade suponha que as soluçôes locais de $P A(t) C(F u ; x)$ estejam todas definidas em $[0, T]$.

Considere o funcional $L$ sobre $C([0, T] ; X)$ dado por:

$$
u \mapsto \int_{0}^{T} V(u(t)) d t+R(u(T))
$$

que é convexo, próprio e sci. Como $I(F) \subset C([0, T] ; X)$ é $w$-compacto e $L$ é w-sci segue que $L$ assume o infimo sobre $I(F)$. Logo, existe $u_{0} \in I(F)$ tal que

$$
\int_{0}^{T} V\left(u_{0}(t)\right) d t+R\left(u_{0}(T)\right) \leq \int_{0}^{T} V(u(t)) d t+R(u(T))
$$

para todo $u \in I(F)$.

Exemplo 2.13 Sejam $X$ um espaço de Banach, $k:[0, T] \times[0, T] \rightarrow L(X, X)$ uma função continua tal que

$$
\|k(t, s)\| \leq M_{1}, \forall t, s \in[0, T] .
$$

Sejam $V:[0, T] \times X \rightarrow X$ uma função contínua e limitada e $F: C([0, T] ; X) \rightarrow L^{1}(0, T ; X)$ o operador definido por:

$$
F(u)(t)=\int_{0}^{t} k(t, s) V(s, u(s)) d s .
$$

Note que $F u \in L^{1}(0, T ; X)$. De fato,

$$
\begin{aligned}
\|F u\|_{1} & =\int_{0}^{T}\left\|\int_{0}^{t} k(t, s) V(s, u(s)) d s\right\| d t \\
& \leq \int_{0}^{T} \int_{0}^{t}\|k(t, s) V(s, u(s))\| d s d t \\
& \leq M_{1} \int_{0}^{T} \int_{0}^{t}\|V(s, u(s))\| d s d t \\
& \leq M_{1} \frac{T^{2}}{2} \sup _{s \in[0, T]}\|V(s, u(s))\|<\infty .
\end{aligned}
$$

$A$ desigualdade acima mostra que $F$ é também limitado, pois $V$ é limitada. Além disso, $F$ é continuo, pois dado $\varepsilon>0$, da continuidade de $V$ existe $\delta>0$ tal que se $\left|s_{1}-s_{2}\right|+\left\|u_{1}-u_{2}\right\|<$ $\delta$, entẫo $\left\|V\left(s_{1}, u_{1}\right)-V\left(s_{2}, u_{2}\right)\right\|<\varepsilon$. Portanto, se $\|u-v\|_{\infty}<\delta$, então

$$
\begin{aligned}
\|F u-F v\|_{1} & =\int_{0}^{T}\|F u(t)-F v(t)\| d t \\
& =\int_{0}^{T}\left\|\int_{0}^{t} k(t, s)(V(s, u(s))-V(s, v(s))) d s\right\| d t \\
& \leq M_{1} \int_{0}^{T} \int_{0}^{t}\|V(s, u(s))-V(s, v(s))\| d s d t \\
& <M_{1} \varepsilon \frac{T^{2}}{2} .
\end{aligned}
$$


Temos ainda, $F$ aplica subconjuntos limitados em subconjuntos uniformemente integráveis. Para ver isto, tomemos $B \subset C([0, T] ; X)$ limitado, $\varepsilon>0$ e $E \subset[0, T]$ mensurável. Entâo, para $u \in B$,

$$
\begin{aligned}
\int_{E}\|F u(t)\| d t & =\int_{E}\left\|\int_{0}^{t} k(t, s) V(s, u(s)) d s\right\| d t \\
& \leq \int_{E} \int_{0}^{t} M_{1}\|V(s, u(s))\| d s d t \leq M_{1} \operatorname{LTm}(E)
\end{aligned}
$$

para todo $u \in B$, e alguma constante

$$
\infty>L=L(B) \geq \sup _{s \in[0, T], u \in B} V(s, u(s))
$$

Tomando $0<\delta<\frac{\varepsilon}{M_{1} L T}$ e $m(E)<\delta$, concluímos que $F(B)$ é um subconjunto uniformemente integrável.

$N a$ verdade temos que $(F u) \in C([0, T] ; X)$. De fato, dado $\varepsilon>0$ e $t_{0} \in[0, T]$ da continuidade de $k$ obtemos $\delta_{1}>0$, e como $V(\cdot, u(\cdot)) \in L^{1}(0, T ; X)$ existe $\delta_{2}>0$ tal que (supondo $\left.0 \leq t_{0} \leq t\right)$

$$
\int_{t_{0}}^{t}\|V(s, u(s))\| d s<\varepsilon e\left\|k(t, s)-k\left(t_{0}, s\right)\right\|<\varepsilon .
$$

Logo, tomando $\delta=\min \left\{\delta_{1}, \delta_{2}\right\}$, obtemos

$$
\begin{aligned}
\left\|F u(t)-F u\left(t_{0}\right)\right\| & \leq \int_{0}^{t_{0}}\left\|k(t, s)-k\left(t_{0}, s\right)\right\|\|V(s, u(s))\| d s \\
& +\int_{t_{0}}^{t}\|k(t, s)\| V(s, u(s)) \| d s \\
& \leq \varepsilon\|V(\cdot, u(\cdot))\|_{1}+M_{1} \int_{t_{0}}^{t}\|V(s, u(s))\| d s \\
& <\varepsilon\|V(\cdot, u(\cdot))\|_{1}+M_{1} \varepsilon \\
& =\varepsilon\left(M_{1}+\|V(\cdot, u(\cdot))\|_{1}\right) .
\end{aligned}
$$

Logo, $(F u)$ é uma funçâo contínua.

Este operador mostra que problemas do tipo $P A(t) C(F u ; x)$ assumem os mais variados tipos, neste caso, uma inclusão integro-diferencial. Tais problemas são de interesse prático, pois aparecem em muitas situações Físicas. Quando o operador $F$ depende de instantes anteriores estes problemas aparecem na Mecância do Contínuo e no estudo da elasticidade e da condução de calor em materiais com memória. Apresentamos uma aplicação no exemplo 2.24 .

Para estes problemas a técnica geralmente usada para provar a existência de solução é o Método de Rothes. Veja Kacur LNM 1192 ou K. Rektorys, The Method of Discretization in Time and Partial Differential Equations, Dordrecht-Boston-London, (1982). Veja também Miller, an integro-differential equation for rigid heat conductors with memory, J. Math. Anal 66(1978), 313-332.

Com frequência, uma equação diferencial parcial pode ser escrita na forma de um problema abstrato com o operador $A$ acretivo. É o que os dois exemplos abaixo ilustram. 
Exemplo 2.14 Considere a equação

$$
\left\{\begin{array}{l}
u_{t}+(g(u))_{x}=0, t>0,0<x<1, \\
u(0, x)=u_{0}(x), 0<x<1 \\
u(t, 0)=0, t>0
\end{array}\right.
$$

onde $g: \mathbf{R} \rightarrow \mathbf{R}$ é contínua, estritamente crescente, $g(0)=0$ e $g(\mathbf{R})=\mathbf{R}$.

Sejam $X=L^{1}([0,1])$ e $A u=(g(u))^{\prime}$ para todo $u \in D(A)$, onde

$$
D(A)=\{u \in C([0,1]) ; u(0)=0 \text { e } g(u) \text { abs. contínua }\} .
$$

Sabemos de [38] que A é m-acretivo em $X$ e que $\overline{D(A)}=X$. Assim o problema acima pode ser reduzido a um problema abstrato de Cauchy:

$$
\left\{\begin{array}{l}
\frac{d u}{d t}+A u \ni 0, t>0 \\
u(0)=u_{0}
\end{array}\right.
$$

onde $u:[0, \infty) \rightarrow X$ é a função procurada e $\frac{d u}{d t}$ é a derivada da função u com valores em $X$.

Segue do Teorema 2.7 que (*) tem uma solução integral local.

Exemplo 2.15 A equação da onda também pode ser escrita na forma de problema abstrato. Este exemplo é devido a Brezis [10].

Seja $\Omega \subset \mathbf{R}^{N}$ um aberto limitado com fronteira $\Gamma$. Procuramos uma funçáa $u:[0, \infty) \times$ $\bar{\Omega} \rightarrow \mathbf{R}$ que seja solução da equação das ondas:

$$
\begin{cases}\frac{\partial^{2} u}{\partial t^{2}}-\Delta u=0 & \text { em } \Omega \\ u=0 & \text { em } \Gamma \times(0, \infty) \\ u(0, x)=u_{0}(x) & \text { em } \Omega \\ \frac{\partial u}{\partial t}(0, x)=v_{0}(x) & \text { em } \Omega\end{cases}
$$

Fixado $t \geq 0, u(t)$ denotará a função $x \mapsto u(t, x)$. Fazendo $v=\frac{\partial u}{\partial t}, U=\left[\begin{array}{l}u \\ v\end{array}\right] e$ $U_{0}=\left[\begin{array}{l}u_{0} \\ v_{0}\end{array}\right]$ e $A=\left[\begin{array}{cc}0 & -I \\ -\Delta & 0\end{array}\right]$, o problema acima pode ser escrito na forma

$$
\left\{\begin{array}{l}
\frac{d U}{d t}+A U=0, t \geq 0 \\
U(0)=U_{0}
\end{array}\right.
$$

Tomando $H=H_{0}^{1}(\Omega) \times L^{2}(\Omega)$ munido do produto interno

$$
\left(U_{1}, U_{2}\right)=\int_{\Omega} \nabla u_{1} \cdot \nabla u_{2} d x+\int_{\Omega} v_{1} v_{2} d x
$$


onde $U_{i}=\left[\begin{array}{l}u_{i} \\ v_{i}\end{array}\right]$.

$O$ dominio de $A$ é $D(A)=\left(H^{2}(\Omega) \cap H_{0}^{1}(\Omega)\right) \times H_{0}^{1}(\Omega)$ e prova-se que A é monótono maximal em H. Pelo Teorema 2.7 existe uma solução local integral para a equação da onda.

Um exame rápido na prova do Teorema 2.7 mostra que para $F \equiv 0$, a solução local integral obtida do Teorema 2.7 , existe em todo intervalo $[0, T]$. Este é o caso dos exemplos 2.14 e 2.15 .

Vamos apresentar um resultado clássico sobre existência de solução.

Corolário 2.16 Seja $A(t), 0 \leq t \leq T$, uma familia de operadores $m$-acretivos sobre um espaço de Banach $X$ que satisfaz $\mathbf{H 1}$ e H2. Seja $F$ um operador em $C([0, T] ; X)$ tal que

$$
\|F u(t)-F v(t)\| \leq c t\|u-v\|_{\infty} .
$$

Então, o problema PA(t)C(Fu,x) tem uma solução local.

Prova: A hipótese implica que $F$ leva subconjuntos limitados em uniformemente integráveis, e portanto, dado $\varepsilon>0$, existe $\delta>0$ tal que

$$
T_{\delta} B_{x} F E_{\delta}\left(C_{\varepsilon}^{\delta}\right) \subset C_{\varepsilon}^{\delta} .
$$

Além disso, para $u$ e $v$ em $C_{\varepsilon}^{\delta}$ temos

$$
\left\|B_{x} F(u)(t)-B_{x} F v(t)\right\| \leq \int_{0}^{t}\|F u(s)-F v(s)\| d s .
$$

Segue que

$$
\left\|B_{x} F(u)(t)-B_{x} F(v)(t)\right\| \leq \frac{c t^{2}}{2}\|u-v\|_{\infty} .
$$

e portanto, por indução,

$$
\left\|\left(B_{x} F\right)^{n} u-\left(B_{x} F\right)^{n} v\right\|_{\infty} \leq \frac{c T^{n+1}}{(n+1) !}\|u-v\|_{\infty},
$$

para todo inteiro positivo $n$.

Logo, para $n$ suficientemente grande tem-se que

$$
\frac{c T^{n+1}}{(n+1) !}<1
$$

e $\operatorname{assim}\left(T_{\delta} B_{x} F E_{\delta}\right)$ tem um ponto fixo. 


\subsection{Existência global}

Nesta secção apresentaremos um resultado simples de existência global de solução integral para o problema $P A(t) C(F u ; x)$. Na prova utilizamos argumentos usuais.

Seja $A(t), 0 \leq t \leq T$, uma família de operadores $m$-acretivos satisfazendo $\mathbf{H} 1$ e $\mathbf{H} 2$. Seja $F$ um operador em $C([0, T] ; X)$ contínuo e limitado satisfazendo

$$
\|F(u)\|_{\infty} \leq c\|u\|_{\infty}
$$

para todo $u \in C([0, T] ; X)$. Suponha também que para cada $T>0$ o problema $P A(t) C(F u ; x)$ admite uma solução integral $u$ definida em $\left[0, T_{\max }\right) \subset[0, T]$ e que

$$
\limsup _{t \rightarrow T_{\max }}\|u(t)\|=+\infty \text {. }
$$

Teorema 2.17 Nas condições acima, PA(t)C(Fu;x) admite uma solução global.

Prova: Seja $\left[0, T_{\max }\right) \subset[0, T]$ o intervalo máximo de definição da solução integral $u$ e suponhamos que $T_{\max }<T$. Seja $0<h<\frac{1}{c}$ e $v$ solução integral do problema

$$
\left\{\begin{array}{l}
\frac{d v}{d t}+A(t) v(t) \ni 0, \quad T_{\max }-h \leq t \leq T, \\
v\left(T_{\max }-h\right)=u\left(T_{\max }-h\right)
\end{array}\right.
$$

Então, para cada $T_{\max }-h \leq t<T_{\max }$, temos

$$
\|u(t)-v(t)\| \leq\left\|u\left(T_{\max }-h\right)-v\left(T_{\max }-h\right)\right\|+\int_{T_{\max }-h}^{t}\|(F u)(\sigma)\| d \sigma
$$

Como $t \mapsto v(t)=U\left(t, T_{\max }-h\right) u\left(T_{\max }-h\right)$ é contínua em $\left[T_{\max }-h, T_{\max }\right]$, pois $U$ é o operador de evolução, seja.

$$
c_{1}=\sup _{t \in\left[T_{\max }-h, T_{\max }\right]}\left\|U\left(t, T_{\max }-h\right) u\left(T_{\max }-h\right)\right\| .
$$

Logo,

$$
\left\|u(t)-U\left(t, T_{\max }-h\right) u\left(T_{\max }-h\right)\right\| \leq 0+\int_{T_{\max }-h}^{t}\|(F u)(\sigma)\| d \sigma
$$

e portanto

$$
\|u(t)\| \leq \int_{T_{\max }-h}^{t}\|(F u)(\sigma)\| d \sigma+\left\|U\left(t, T_{\max }-h\right) u\left(T_{\max }-h\right)\right\| .
$$

Se $l=\sup \left\{\|u(\sigma)\|, \sigma \in\left[T_{\text {max }}-h, t\right]\right\}$, segue que $l \leq c_{1}+c l h$, e portanto $l \leq \frac{c_{1}}{1-c h}$ e assim $u$ é limitada contrariando a nossa hipótese. Logo, $u$ está definida em $[0, T]$, o que conclui a prova. 


\subsection{Propriedades do conjunto de soluções}

Agora estudaremos propriedades do conjunto de soluções

$$
I(N)=\bigcup_{F \in N} I(F)
$$

dos problemas $P A(t) C(F u ; x)$ onde $N$ é como em $\mathbf{H 3}$.

Estamos supondo que as soluções locais dos problemas $P A(t) C(F u ; x), F \in N$, estão todas definidas em um intervalo comum que pode ser admitido igual a $[0, T]$.

Teorema 2.18 Sejam $A(t), 0 \leq t \leq T$, uma família de operadores $m$-acretivos satisfazendo $\mathbf{H 1}$ e $\mathbf{H 2}$, e $N$ como em H3. Suponha que todo $F \in N$ satisfaz a pelo menos uma das condições $\mathbf{H 4}$, $\mathbf{H 5}$ ou $\mathbf{H 6}$. Se toda seç̧ão

$$
I(N)(t)=\left\{u_{F}(t): u_{F} \in I(F), t \in[0, T], F \in N\right\}
$$

é relativamente compacto, então $I(N)$ é equicontínuo.

Prova:Dividimos a prova em três partes.

Primeiro caso: $t=0$

Seja $u_{0}$ a solução integral de $\operatorname{PA}(t) \mathrm{C}(0 ; x)$, isto é,

$$
u_{0}(t)=U(t, 0) x .
$$

Então, para $u_{F} \in I(F)$,

$$
\begin{aligned}
& \left\|u_{F}(t)-x\right\| \leq\left\|u_{F}(t)-U(t, 0) x\right\|+\|U(t, 0) x-x\| \leq \\
& \leq\|U(t, 0) x-x\|+\left\|u_{F}(0)-x\right\|+\int_{0}^{t}\left\|F\left(u_{F}\right)(s)\right\| d s,
\end{aligned}
$$

pois $U(\cdot, 0) x$ e $u_{F}$ são soluções integrais e $F\left(u_{F}\right)$ está em $C([0, T] ; X)$.

Como $U$ é o operador de evolução associado à família $A(t), 0 \leq t \leq T$, então $U$ é contínuo. Assim, dado $\varepsilon>0$ existe $\delta^{\prime}>0$ tal que se $0 \leq t<\delta^{\prime}$, então

$$
\|U(t, 0) x-x\|<\frac{\varepsilon}{2} .
$$

Como $I(N)$ é limitado, então segue de $\mathbf{H 3}$ que existe $\delta_{0}>0$ tal que se $m(E)<\delta_{0}$,

$$
\int_{E}\|F(u)(s)\| d s<\frac{\varepsilon}{2}, \forall F \in N, u \in I(N) .
$$

Tomemos $0<\delta \leq \min \left\{\delta^{\prime}, \delta_{0}\right\}$ e obtemos para $0 \leq t<\delta$ que

$$
\left\|u_{F}(t)-x\right\|<\varepsilon, \forall F \in N .
$$

Isto prova que $I(N)$ é equicontínuo em $t=0$. 
Segundo caso: $0<t<T$

Seja $\delta>0$ tal que $t \pm \delta \in[0, T]$. Para $0<h<\delta, u_{F} \in I(F)$ e $u_{F_{1}} \in I\left(F_{i}\right) \operatorname{com} F_{i} \in N$, temos

$$
\begin{aligned}
\left\|u_{F}(t+h)-u_{F}(t)\right\| & \leq\left\|u_{F}(t+h)-u_{F_{i}}(t+h)\right\| \\
& +\left\|u_{F_{i}}(t+h)-u_{F_{i}}(t)\right\| \\
& +\left\|u_{F_{i}}(t)-u_{F}(t)\right\| .
\end{aligned}
$$

Como $u_{F}$ e $u_{F_{i}}$ são soluções integrais, temos que o primeiro termo da desigualdade acima pode ser majorado por

$$
\left\|u_{F}(t-\delta)-u_{F_{i}}(t-\delta)\right\|+\int_{t-\delta}^{t+h} \| F\left(u_{F}\right)(s)-F_{i}\left(u_{F_{i}}(s) \| d s\right.
$$

e o terceiro termo por

$$
\left\|u_{F}(t-\delta)-u_{F_{i}}(t-\delta)\right\|+\int_{t-\delta}^{t}\left\|F\left(u_{F}\right)(s)-F_{i}\left(u_{F_{i}}\right)(s)\right\| d s .
$$

Logo,

$$
\begin{aligned}
\left\|u_{F}(t+h)-u_{F}(t)\right\| & \leq 2\left\|u_{F}(t-\delta)-u_{F_{i}}(t-\delta)\right\| \\
& +\int_{t-\delta}^{t+\delta}\left\|F\left(u_{F}\right)(s)-F_{i}\left(u_{F_{i}}\right)(s)\right\| d s \\
& +\int_{t-\delta}^{t}\left\|F\left(u_{F}\right)(s)-F_{i}\left(u_{F_{i}}\right)(s)\right\| d s \\
& +\left\|u_{F_{i}}(t+h)-u_{F_{i}}(t)\right\| .
\end{aligned}
$$

Como $I(N(t))$ é relativamente compacto, dado $\varepsilon>0$ existe $0<\delta_{1} \leq \delta$ e $\left\{F_{1}, \ldots, F_{n_{\varepsilon}}\right\} \subset$ $N$ tais que para todo $F \in N$, existe $1 \leq i \leq n_{\varepsilon}$ com

$$
\left\|u_{F}(t-\delta)-u_{F_{i}}(t-\delta)\right\|<\frac{\varepsilon}{2}
$$

Como $N(I(N))$ é uniformemente integrável, existe $0<\delta_{2} \leq \delta_{1}$ tal que

$$
\int_{E}\|F(u)(s)\| d s<\frac{\varepsilon}{2}
$$

sempre que $m(E)<\delta_{2}$ e $F \in N$.

Da continuidade de $u_{F_{i}}$ obtemos $0<\delta_{3} \leq \delta_{2}$ tal que

$$
\left\|u_{F_{\mathrm{i}}}(t+h)-u_{F_{\mathrm{i}}}(t)\right\|<\varepsilon,
$$

se $0<h \leq \delta_{3}$.

Logo,

$$
\left\|u_{F}(t+h)-u_{F}(t)\right\|<5 \varepsilon .
$$

Isto prova que $I(N)$ é equicontínuo em $t \in(0, T)$. 


\section{Terceiro caso: $t=T$}

Procedendo como no caso anterior, dado $\varepsilon>0$, obtemos da compacidade de $\overline{I(N(t))}$, da continuidade das soluções e da integrabilidade uniforme de $N(I(N))$, um elemento $F_{i} \in N$ e um $\delta>0$ tal que para $0<h \leq \delta$, temos

$$
\begin{aligned}
\left\|u_{F}(T-h)-u_{F}(T)\right\| & \leq\left\|u_{F}(t-h)-u_{F_{i}}(T-h)\right\| \\
& +\left\|u_{F_{i}}(T-h)-u_{F_{i}}(T)\right\| \\
& +\left\|u_{F_{i}}(T)-u_{F}(T)\right\| \\
& \leq 2\left\|u_{F}(T-\delta)-u_{F_{i}}(T-\delta)\right\| \\
& +\int_{T-\delta}^{T-h}\left\|F u_{F}(s)-F_{i} u_{F_{i}}(s)\right\| d s \\
& +\int_{T-\delta}^{T}\left\|F u_{F}(s)-F_{i} u_{F_{i}}(s)\right\| d s \\
& +\left\|u_{F_{i}}(T-h)-u_{F}(T)\right\| \\
& \leq 5 \varepsilon .
\end{aligned}
$$

Logo, $I(N)$ é equicontínuo em $T$ e portanto $I(N)$ é equicontínuo em $[0, T]$.

Pelo Teorema de Ascoli-Arzela o teorema 2.18 pode ser reafirmado como:

Corolário $2.19 I(N)$ é relativamente compacto se, e somente se, $I(N)(t)$ é relativamente compacto para cada $t \in[0, T]$.

Mas quando $I(N)(t)$ será relativamente compacto? Uma resposta é dada no próximo teorema. Precisaremos de uma hipótese adicional:

H7- A família $A(t), 0 \leq t \leq T$, gera um operador de evolução compacto.

Teorema 2.20 Se $A(t), 0 \leq t \leq T$ satisfaz $\mathbf{H 7}$ e as hipóteses do Teorema 2.18, então toda secção $I(N)(t)$ é relativamente compacta.

Prova: $O$ caso $t=0$ é obvio. Logo, basta provar para $t \in(0, T]$. Iniciamos observando que: Sejam $U(t, 0) x=u_{0}(t)$ a solução integral de $P A(t) C(0 ; x)$ e $u_{F}$ solução de $P A(t) C(F u ; x)$, então

$$
\left\|U(t, 0) x-u_{F}(t)\right\| \leq\left\|u_{F}(s)-U(s, 0) x\right\|+\int_{s}^{t}\left\|F u_{F}(\tau)\right\| d \tau,
$$

$0 \leq s \leq t \leq T$.

Como $\bar{K}=I(N) \subset C([0, T] ; X)$ é limitado, dado $\varepsilon>0$ existe $\delta>0$ tal que se $m(E)<\delta$, então

$$
\int_{E}\|F u(\tau)\| d \tau<\varepsilon, \forall F \in N e u \in K .
$$

Como $I(N)(s) \subset \overline{D(A(s))}$ é limitado e o operador de evolução é compacto, então

$$
U(t, s) I(N)(s), 0 \leq s \leq t \leq T,
$$

é relativamente compacto. 
Tomemos $t \in(0, T]$. Então, existe $0<2 h<\delta$ tal que $(t-2 h) \in[0, T]$. Para $u_{F}$ solução de $P A(t) C(F u ; x)$, defina:

$$
v^{h}(\tau)=U(\tau+h, t-h) u_{F}(t-h), \tau \in[t-2 h, t]
$$

que é solução integral de $P A(t) C\left(0 ; u_{F}(t-h)\right)$. Logo, vale a seguinte relação:

$$
\left\|v^{h}(t)-u_{F}(t)\right\| \leq\left\|v^{h}(t-2 h)-u_{F}(t-2 h)\right\|+\int_{t-2 h}^{t}\left\|F u_{F}(\tau)\right\| d \tau,
$$

isto é,

$$
\begin{aligned}
\left\|U(t+h, t-h) u_{F}(t-h)-u_{F}(t)\right\| & \leq\left\|U(t-h, t-h) u_{F}(t-h)-u_{F}(t-2 h)\right\| \\
& +\int_{t-2 h}^{t}\left\|F u_{F}(\tau)\right\| d \tau .
\end{aligned}
$$

Da continuidade de $U$, obtemos $0<\delta^{\prime} \leq \delta$ tal que

$$
\left\|U(t-h, t-h) u_{F}(t-h)-u_{F}(t-2 h)\right\|<\varepsilon
$$

usando a integrabilidade uniforme temos que

$$
\left\|U(t+h, t-h) u_{F}(t-h)-u_{F}(t)\right\|<2 \varepsilon, \forall F \in N \text { e } u_{F} \in I(N) .
$$

Logo,

$$
\lim _{h \backslash 0} U(t+h, t-h) u_{F}(t-h)=u_{F}(t),
$$

uniformemente para cada $F \in N$ e $u_{F} \in I(F)$.

Isto sugere definir a seguinte família de operadores compactos:

$$
I(N)(t) \ni u_{F}(t) \stackrel{T^{h}}{\longrightarrow} U(t+h, t-h) u_{F}(t-h) \in X
$$

para $0<h<\delta^{\prime}$. Tomando o limite para $h$ tendendo a zero, obtemos $T^{0}=i_{I(N)(t)}$, a inclusão de $I(N)(t)$ em $X$. Pelo Teorema 1.51, $I(N)(t)$ é relativamene compacto. Isto conclui a prova.

Corolário 2.21 Sob as hipóteses $\mathbf{H 1}, \mathrm{H} 2$ e $\mathbf{H} 7$ o conjunto solução $I(M)$ é relativamente compacto, para todo $M \subset C([0, T] ; X)$ uniformemente integrável.

Prova: Dado $M \subset C([0, T] ; X)$ uniformemente integrável e $f \in M$ definimos $F$ o operador constante

$$
F u \equiv f, \quad \forall u \in C([0, T] ; X) .
$$

Seja $N$ o conjunto destes operadores. Dado $x \in D=\overline{D(A(0))}$, o operador $B_{x} F$, para cada $F \in N$, satisfaz $\mathbf{H 3}, \mathbf{H} 4, \mathbf{H} 5$ e H6. Pelo Teorema 2.20 o conjunto $I(N)$ é relativamente compacto. 
Teorema 2.22 Seja $A(t), 0 \leq t \leq T$, uma família de operadores $m$-acretivos satisfazendo H1, H2 e H7. Seja F : $\bar{C}([0, T] ; X) \rightarrow C([0, T] ; X)$ um operador contínuo limitado e causal. Então, o problema $P A(t) C(F u ; x)$ tem pelo menos uma soluçâo local e o conjunto das soluções $I(F)$ é compacto.

Prova: A idéia da prova é usar os Teoremas 2.7 e 2.20 .

Seja $K \subset C([0, T] ; X)$ limitado. Dados $f \in F(K)$ e $E \subset[0, T]$ mensurável, temos

$$
\int_{E}\|f(t)\| d t=\int_{E} 1 .\|f(t)\| d t \leq\|f\|_{\infty} m(E) \leq M m(E)
$$

onde $M=\sup \left\{\|f\|_{\infty}: f \in F(K)\right\}$.

Logo, $F(K)$ é subconjunto uniformemente integrável. Usando o Corolário $2.21 \mathrm{com}$ $M=F(K)$, obtemos que $I(F(K))$ é relativamente compacto, isto é, $B_{x}(F(K))=B_{x} F(K)$ é relativamente compacto. Como o operador de Bénilan-Pavel $B_{x}$ é contínuo, segue que $\left(B_{x} F\right)$ leva subconjuntos limitados em subconjuntos relativamemente compactos. Segue do Teorema 2.7 que $P A(t) C(F u ; x)$ tem conjunto solução $I(F)$ fechado e não vazio, portanto, pelo teorema $2.20 I(F)$ é compacto.

O Teorema 2.22 generaliza, para o caso de uma família $A(t), 0 \leq t \leq T$, um resultado de Vrabie [60] que provou a existência de solução para $P A C(F u ; x)$, com

$$
F: C([0, T] ; X) \rightarrow L^{p}(0, T ; X), 1<p \leq \infty
$$

Veja o Corolário 3.3

Usando o Teorema 2.20 podemos reformular o exemplo 2.12. De fato, podemos retirar as hipóteses de convexidade e de compacidade fraca.

Exemplo 2.23 Como anteriormente, consideremos o problema de controle

$$
\inf _{u \in I(F)} \int_{0}^{T} V(u(t)) d t+R(u(T))
$$

sujeito ao seguinte problema abstrato de Cauchy

$$
\left\{\begin{array}{l}
\frac{d u}{d t}+A(t) u(t) \ni(F u)(t), 0 \leq t \leq T \\
u(0)=0 \in \overline{D(A(0))}
\end{array}\right.
$$

Estamos admitindo que as soluções do problema de Cauchy estejam todas definidas no intervalo $[0, T]$ e que $V, R: X \rightarrow \mathbf{R}^{+} \cup\{\infty\}$ sejam próprias e sci.

Suponha que exista $u_{F} \in I(F)$ tal que

- $V\left(u_{F}(\cdot)\right) \in L^{1}\left(0, T ; \mathbf{R}^{+}\right)$

- $R(u(T))<\infty$. 
Suponha também que $N=\{F\}$ satisfaz $\mathbf{H 3}$ e que $\left(B_{x} F\right)$ satisfaz $\mathbf{H} 4$ ou $\mathbf{H} 5$ ou $\mathbf{H 6}$, e que $A(t), 0 \leq t \leq T$, satisfaz $\mathbf{H 1}, \mathbf{H} 2$ e $\mathbf{H} 7$.

Nestas condições afirmamos que o problema de controle admite pelo menos um controle ótimo. Com efeito, o funcional $L$ sobre $C([0, T] ; X)$ dado por

$$
u \mapsto \int_{0}^{T} V(u(t)) d t+R(u(T))
$$

é sci e $I(F)$ é compacto pelo Teorema 2.20. Segue que existe $u_{0} \in I(F)$ tal que

$$
L\left(u_{0}\right) \leq L(u), \forall u \in I(F) .
$$

Exemplo 2.24 O modelo Físico de condução de calor em uma barra ou de vibraçâo de uma membrana de materiais com memória pode ser escrito na forma abaixo. Veja [3] ou [46]. Vamos provar que o seguinte problema

$$
\left\{\begin{array}{l}
\frac{d u}{d t}+A(t) u(t) \ni \int_{0}^{t} k(t-s) g(s, u(s)) d s, 0 \leq t \leq T \\
u(0)=x \in D,
\end{array}\right.
$$

onde $A(t), 0 \leq t \leq T$ é uma familia de operadores $m$-acretivos em um espaço de Banach $X, g:[0, T] \times D \rightarrow X$ e $k:[0, T] \rightarrow L(X)$ são contínuas, admite pelo menos uma soluçâo integral local.

Temos o seguinte resultado:

Sejam $A(t), 0 \leq t \leq T$, uma família de operadores $m$-acretivos que satisfazem H1, H2 e H7 e $g$ e $k$ como acima. Então, para cada $x \in D$ existe $c \in(0, T]$ tal que o problema $(*)$ tem pelo menos uma solução integral em $[0, c]$.

De fato, seja $u_{0}$ a solução integral do problema homogêneo associado a (*). Tomemos $\rho>0, c_{0} \in(0, T]$ e $M>0$ tal que

$$
\begin{gathered}
B(x ; \rho)=\{v \in X:\|v-x\| \leq \rho\} \\
\|g(s, v)\| \leq M
\end{gathered}
$$

$s \in\left[0, c_{0}\right], v \in D \cap B(x ; \rho) \mathrm{e}$

$$
\left\|u_{0}(t)-x\right\|+c_{0} M=\|U(t, 0) x-x\|+c_{0} M \leq \rho,
$$

$t \in\left[0, c_{0}\right]$, onde $\{U(t, s): U(t, s): D \rightarrow D, 0 \leq s \leq t \leq T\}$ é um operador de evolução associado à família $A(t), 0 \leq t \leq T$.

Escolha $c_{1} \in\left(0, c_{0}\right]$ e $r \in(0, M]$ tal que

$$
c_{1} \sup _{\tau \in[0, T]}\|k(\tau)\| M \leq r,
$$

e defina

$$
C_{1}=\left\{f \in C\left(\left[0, c_{1}\right] ; X\right):\|f(t)\| \leq r, t \in\left[0, c_{1}\right]\right\}
$$


que é não vazio, convexo, limitado e fechado em $C\left(\left[0, c_{1}\right] ; X\right)$. Como $C_{1}$ é uniformemente integrável, pelo Corolário 2.21 existe $c \in\left(0, c_{1}\right]$ tal que o conjunto de soluções integrais $B_{x}\left(C_{1}\right)$, vistas como funções restritas ao intervalo $[0, c]$, é relativamente compacto em $C([0, c] ; X)$.

Defina $C=\{f \in C([0, c] ; X):\|f(t)\| \leq r, t \in[0, c]\}$. Seja $P: D(P) \subseteq C \rightarrow C([0, c] ; X)$ dado por $P f(t)=\int_{0}^{t} k(t-s) g\left(s, u_{f}(s)\right) d s$, onde $u_{f}$ é a única solução integral do problema $P A(t) C(f ; x) \mathrm{e}$

$$
D(P)=\left\{f \in C: u_{f} \text { é a solução integral de } P A(t) C(f ; x)\right\} .
$$

Observamos que se $P f=f$, então $u_{f}$ é solução integral de $(*)$. Para completar a prova é suficiente mostrar que $P$ tem pelo menos um ponto fixo em $C$. Afirmamos que $D(P)=C$. De fato, dado $f \in C$ temos

$$
\begin{aligned}
\left\|u_{f}(t)-x\right\| & \leq\left\|u_{f}(t)-U(t, 0) x\right\|+\|U(t, 0) x-x\| \\
& \leq\|U(t, 0) x-x\|+\int_{0}^{c}\|f(s)\| d s \\
& \leq\|U(t, 0) x-x\|+c r \leq \rho
\end{aligned}
$$

$\operatorname{assim} u(t) \in D \cap B(x ; \rho)$ e portanto $D(P)=C$.

Vemos também que $P(C) \subset C$, pois para $f \in C$, temos que

$$
\begin{aligned}
\|P f(t)\| & \leq \int_{0}^{t}\|k(t-s)\|\left\|g\left(s, u_{f}(s)\right)\right\| d s \\
& \leq c \sup _{\tau \in[0, T]}\|k(\tau)\| M \leq r,
\end{aligned}
$$

para todo $t \in[0, c]$. Segue que $P f \in C$.

$\mathrm{O}$ operador $P$ pode ser fatorado como $P=F B_{x}$, onde

$$
B_{x}: C \rightarrow C([0, c] ; X)
$$

é o operador de Bénilan-Pavel e $F: C([0, c] ; X) \rightarrow C([0, c] ; X)$ é dado por

$$
F u(t)=\int_{0}^{t} k(t-s) g(s, u(s)) d s .
$$

Como $F$ e $B_{x}$ são contínuos, então $P$ é contínuo de $C$ em si mesmo, $C$ munido da topologia forte induzida de $C([0, c] ; X)$.

Defina $K=\overline{\operatorname{co} P(C)}$. Observamos que $K \subseteq C$ e portanto $B_{x}(K)$ é relativamente compacto em $C([0, c] ; X)$. Como $C([0, c] ; X)$ é separado e $F$ é contínuo, temos que $F B_{x}(K)=$ $P(K)$ é relativamente compacto em $C([0, c] ; X)$. Pelo Teorema de Schauder $P$ tem um ponto fixo em $K$. Isto é, existe $f \in K$ e portanto em $C$, tal que $P f=f$. Ou seja $B_{x} f$ é solução integral em $[0, c]$. 


\subsection{Um Resultado de convergência}

$\mathrm{Na}$ primeira secção vimos que sob certas condições o operador $\left(T_{r} B_{x} F E_{r}\right)$ aplica $C_{\varepsilon}^{r}$ em $C_{\varepsilon}^{\tau}$, para algum $0<r \leq T$. Agora veremos que um procedimento numérico pode ser utilizado para gerar uma sequência de funções contínuas $\left(u_{n}\right)$ que converge forte para uma solução do problema abstrato de Cauchy $P A(t) C(F u ; x)$.

Definição 2.25 Uma aplicação multivalente $A: X \rightarrow 2^{X}$ sobre um espaço métrico completo $(X, d)$ é chamada localmente Lipschitz contínua em $x_{0} \in X$, com módulo a, se em alguma vizinhança de $x_{0}$,

$$
f_{x_{0}}^{A}(x):=\sup _{z \in A x} d\left(z, A x_{0}\right) \leq a d\left(x, x_{0}\right) .
$$

Note que $A$ é semicontínua superiormente em $x_{0}$ se, e somente se, $f_{x_{0}}^{A}$ é contínua em $x_{0}$. $\mathrm{O}$ resultado de convergência é uma consequência simples de um teorema devido a Khang. A idéia é usar o teorema de Khang do Capítulo 1 para determinar pontos fixos de $\left(T_{r} B_{x} F E_{r}\right)$, que serão soluções locais.

Teorema 2.26 Sejam $A(t), 0 \leq t \leq T$, uma família de operadores m-acretivos satisfazendo H1 e $\mathbf{H 2}$ e $N=\{F\}$ uma aplicação contínua e limitada como em H3. Suponha ainda que $\left(T_{r} B_{x} F E_{r}\right)$ seja nấ-expansiva, que o conjunto $V$ de seus pontos fixos seja não-vazio e que

$$
\left(I-T_{r} B_{x} F E_{r}\right)^{-1}
$$

seja localmente Lipschitz contínua na origem com módulo a. Então, para $0<t<1$, a sequência $\left(u_{n}\right)$ dada por

$$
u_{n+1}=(1-t) u_{n}+t\left(T_{r} B_{x} F^{\prime} E_{r}\right) u_{n}, \quad n \geq 1
$$

e $u_{1}=0$, converge fortemente para um ponto fixo de $\left(T_{r} B_{x} F E_{r}\right)$ em $C_{\varepsilon}^{r}$. Além disso, para todo $\varepsilon_{1}>0$ existe $N \in \mathbf{N}$ tal que

$$
d\left(u_{n}, V\right) \leq a \varepsilon_{1}, \forall n \geq N .
$$

Prova:Tomemos

$$
\begin{aligned}
T & =\left(T_{r} B_{x} F E_{r}\right) \\
v_{n} & =T u_{n}-u_{n} \\
w_{n} & =\frac{1}{t}\left(T u_{n+1}-T u_{n}\right) .
\end{aligned}
$$

Então, valem as propriedades:

i) $\left(\left\|v_{n}\right\|_{\infty}\right)$ é não-crescente.

2i) $\left\|w_{n}\right\|_{\infty} \leq\left\|v_{n}\right\|_{\infty}$

$3 \mathrm{i}) v_{n+1}=(1-t) v_{n}+t w_{n}$.

Usando o resultado 1.52 de D. B. Khang $\operatorname{com} M=\frac{1}{t} \operatorname{diam} C_{\varepsilon}^{r}$ e $\varepsilon_{1}>0$ arbitrário, existe $N$ natural tal que

$$
\left\|(I-T) u_{n}\right\|_{\infty}<\varepsilon_{1}, \forall n \geq N \text {. }
$$


Como $V=(I-T)^{-1}(0)$ segue que

$$
d\left(u_{n}, V\right) \leq f_{0}^{(I-T)^{-1}}\left(u_{n}-T u_{n}\right) \leq a\left\|u_{n}-T u_{n}\right\|_{\infty}<a \varepsilon_{1} .
$$

Segue que $\lim u_{n}=u \in V$ e isto termina a prova. $\square$ 


\section{Capítulo 3}

\section{Soluções Lipschitz contínuas}

Neste capítulo procuramos ainda provar a existência de soluções integrais de problemas abstratos de Cauchy para operadores dependentes ou não do tempo. Procuramos demonstrar também a existência de soluções integrais Lipschitz contínuas.

\subsection{Resultados de existência}

Para um operador $A m$-acretivo sobre um espaço de Banach $X, x \in \overline{D(A)}$ e $f \in L^{1}(0, T ; X)$, Bénilan [6] provou a existência e unicidade de soluções integrais para o problema abstrato $P A C(f ; x)$. Pavel estendeu este resultado para problemas do tipo $P A(t) C(f ; x)$. Veja Teorema de Pavel no Capítulo 2.

Já falamos de soluções integrais segundo Pavel, agora vamos recordar o conceito de solução integral devido a Bénilan para $P A C(f ; x)$.

Definição 3.1 Uma solução integral, no sentido de Bénilan, para $P A C\left(f ; x_{0}\right), x_{0} \in \overline{D(A)}$, é uma funçẫo $u \in C([0, T] ; \overline{D(A)})$ tal que $u(0)=x_{0}$ e que

$$
\|u(t)-x\|^{2} \leq\|u(s)-x\|^{2}+2 \int_{s}^{t}\langle f(\tau)-y, u(\tau)-x\rangle_{s} d \tau
$$

para todo $x \in D(A), y \in A x$ e todo $0 \leq s \leq t \leq T$.

Observação 3.2 Note que um operador $A$ m-acretivo sempre satisfaz às hipóteses $\mathbf{H} 1 \epsilon$ H2 do Capítulo 2, com $h \equiv 0$ ou $L \equiv 0$. Neste caso, os dois conceitos de soluçấo integral coincidem. Em [47] Pavel mostrou que sob as condiçôes $\mathbf{H 1}$ e $\mathbf{H} 2$ A gera um semigrupo.

Para um operador acretivo $A$, lembramos que o resolvente de $A$ é $J_{\lambda}=(I+\lambda A)^{-1}$ e a aproximação de Yosida é $A_{\lambda}=\frac{1}{\lambda}\left(I-J_{\lambda}\right)$.

Sejam $|A x|=\lim _{\lambda \rightarrow 0}\left\|A_{\lambda} x\right\|, x \in X, \mathrm{e}$

$$
\hat{D}(A)=\{x \in X:|A x|<\infty\} .
$$

$\hat{D}(A)$ é chamado o domínio generalizado de $A$. Em geral, temos

$$
D(A) \subset \hat{D}(A) \subset \overline{D(A)} \text {. }
$$


Se $x \in \hat{D}(A)$ e $f \in L^{1}(0, T ; X)$ é de variação limitada, então sabemos de [3] que $P A C(f ; x)$ admite uma única solução integral u e esta é Lipschitz contínua em $[0, T]$. Provaremos que o mesmo ainda é verdade para problemas um pouco mais gerais.

Inicialmente consideremos o problema $P A C(F u ; x)$, onde $A$ é um operador $m$-acretivo sobre um espaço de Banach $X, x \in D(A)$ e $F: C([0, T] ; X) \rightarrow C([0, T] ; X)$ operador contínuo. Quando $A(t), 0 \leq t \leq T$, não depende de $t$ podemos admitir que $F u \in L^{1}(0, T ; X)$. É o que diz o corolário abaixo, que é consequência direta do Teorema 2.7 do capítulo anterior

Corolário 3.3 Sejam A um operador $m$-acretivo definido em um espaço de Banach $X$, $F: C([0, T] ; X) \rightarrow L^{1}(0, T ; X)$ contínuo e limitado que aplica subconjuntos limitados em subconjuntos uniformememnte integráveis e $x \in D=\overline{D(A)}$. Suponha ainda que $F$ satisfaz a uma das condiçôes:

H4- $B_{x} F$ é compacto.

H5- Existe $\varphi: C([0, T] ; X) \rightarrow \mathbf{R}^{+}$sci tal que

$$
\left\|v-B_{x} F v\right\|_{\infty} \leq \varphi(v)-\varphi\left(B_{x} F v\right)
$$

para todo $v \in C([0, T] ; X)$.

H6- Existe $\varphi: \mathbf{R}^{+} \rightarrow \mathbf{R}^{+}$scs tal que $\varphi(t)<t e$

$$
\left\|B_{x} F u-B_{x} F v\right\|_{\infty} \leq \varphi(\|u-v\|),
$$

para todos u e $v \in C([0, T] ; X)$.

Então, o problema PAC $(F u, x)$ tem pelo menos uma solução num intervalo $[0, \delta]$. Além disso, o conjunto de soluçôes $I(F)$ é limitado e fechado em $C([0, \delta] ; X)$.

Prova: Seguindo a prova do Teorema 2.7, dados $\varepsilon>0$ e $u_{0}$ a única solução integral de $P A C(0 ; x)$, que existe pelo Teorema 2.4 , definimos como anteriormente $C_{\varepsilon}$ conjunto não vazio, convexo, limitado e fechado em $C([0, T] ; X)$. Para $r \in(0, T]$, escrevemos

$$
C_{\varepsilon}^{r}=T_{r}\left(C_{\varepsilon}\right)
$$

que também é convexo, limitado e fechado.

Considerando o conjunto $S_{\varepsilon}$ da prova do Teorema 2.7 tem-se claramente $E_{r}\left(C_{\varepsilon}^{r}\right) \subset S_{\varepsilon}$. Como $S_{\varepsilon}$ é limitado, segue que $F\left(S_{\varepsilon}\right)$ é uniformemente integrável e assim para o $\varepsilon>0$ dado existe $\delta \in(0, T]$ tal que para $r=\delta$ tem-se

$$
\left(T_{\delta} B_{x} F E_{\delta}\right)\left(C_{\varepsilon}^{\delta}\right) \subset C_{\varepsilon}^{\delta},
$$

pois vale a desigualdade abaixo para funçoẽs de $L^{1}$,

$$
\begin{aligned}
\left\|\left(T_{\delta} B_{x} F E_{\delta} v\right)(s)-T_{\delta} u_{0}(s)\right\| & =\left\|\left(B_{x} F E_{\delta} v\right)(s)-u_{0}(s)\right\| \\
& \leq \int_{0}^{\delta}\left\|F E_{\delta} v(s)\right\| d s \leq \varepsilon .
\end{aligned}
$$

Segue $T_{\delta} B_{x} F E_{\delta}$ tem um ponto fixo, que é uma solução local em $[0, \delta]$.

Também é consequência do Capítulo 2 o seguinte 
Teorema 3.4 Seja A um operador m-acretivo.

1) Se $F: C([0, T] ; X) \rightarrow L^{1}(0, T ; X)$ é contínuo, limitado, aplica subconjuntos limitados em subconjuntos uniformemente integráveis e $\left(B_{x} F\right)$ satisfaz $\mathbf{H 4}$ ou $\mathbf{H} 5$ ou $\mathbf{H 6}$, então o problema PAC $(F u ; x)$ tem um conjunto soluçâa $I(F)$ não-vazio, limitado e fechado.

2) Se A gera um semigrupo compacto, então $\overline{I(N)(t)}$ é compacto para cada $t \in[0, T]$.

3) Se $\overline{I(N)(t)}$ é compacto para cada $t \in[0, T]$, então $\overline{I(N)}$ é compacto.

Denotaremos por $B V([0, T] ; X)$ e $L C([0, T] ; X)$ o conjunto das funções de variação limitada e Lipschitz contínuas de $[0, T]$ em $X$, respectivamente. Lembramos que se $f \in$ $B V([0, T] ; X)$ então $f$ tem apenas uma quantidade enumerável de descontinuidades de primeira espécie. Vamos agora enunciar o primeiro resultado de existência de soluções integrais Lipschitz contínuas.

Teorema 3.5 Sejam A um operador m-acretivo, $x \in \hat{D}(A), B_{x}$ o operador de BénilanPavel e $F: C([0, T] ; X) \rightarrow B V([0, T] ; X)$ contínuo limitado que aplica subconjuntos limitados em subconjuntos uniformemente integráveis. Se $B_{x} F$ satisfaz $\mathbf{H} 4$ ou $\mathbf{H} 5$ ou $\mathbf{H 6}$, então o problema PAC $(F u ; x)$ tem pelo menos uma soluçâo (integral) local Lipschitz contínua.

Prova: A idéia da prova é usar o Teorema 2.7. Pelo Teorema 2.7, dado $\varepsilon>0$ existe $\delta>0$ tal que

$$
\left(T_{\delta} B_{x} F E_{\delta}\right)\left(C_{\varepsilon}^{\delta}\right) \subset C_{\varepsilon}^{\delta}
$$

Como $F E_{\delta}\left(C_{\varepsilon}^{\delta}\right)$ é de variação limitada e como $B_{x} f$ é Lipschitz contínua sempre que $f \in B V$ (veja [47]), então $\left(T_{\delta} B_{x} F E_{\delta}\right)$ tem pelo menos um ponto fixo que é uma solução local Lipschitz contínua.

Ainda neste capítulo provaremos uma extensão deste resultado.

Exemplo 3.6 Sejam $H$ um espaço de Hilbert $e \varphi: H \rightarrow \mathbf{R} \cup\{\infty\}$ uma função convexa, própria e sci. Sabemos que $A=-\partial \varphi$ é um operador acretivo maximal. Veja por exemplo [20]. Brezis provou que se

$$
C_{r}=\{x \in D(\varphi):\|x\| \leq r e \varphi(x) \leq r\}
$$

é compacto em $H$ para todo $r>0$, entâo o semigrupo associado a $A$ é compacto. Vale também a recíproca. Segue do Teorema 3.4 que se $F$ satisfaz $\mathbf{H 3}$ e $B_{x} F$ satisfaz $\mathbf{H} 4$ ou $\mathbf{H 5}$ ou H6, entâo o conjunto soluçâo $\overline{I(F)}$ de PAC $(F u ; x), x \in D(A)$, é compacto. Além disso, as soluçôes locais dadas pelo Teorema 2.7 são soluções fortes, pois $H$ tem a propriedade de Radon-Nikodym. Veja [47].

Corolário 3.7 Se o operador

$$
F(u) \equiv f \in B V([0, T] ; X), \forall u,
$$

então o problema PAC $(f ; x)$ tem uma solução local Lipschitz contínua .

A demonstração é imediata, pois neste caso $B_{x} F$ satisfaz claramente $\mathbf{H} 4$ e $\mathbf{H 6}$, e $F$ satisfaz H3. O resultado segue do Teorema 3.5.

Reobtemos assim um resultado já conhecido, veja [3]. 
Observação 3.8 Se o espaço X tem RNP, então a solução dada no teorema acima é diferenciável em quase todo ponto, sendo portanto uma soluçấo forte.

Para os próximos resultados usaremos a noção de seleçâo de operadores multivalentes introduzida no Capítulo 1.

\subsection{Teorema de existência II}

Teorema 3.9 Seja $A: X \rightarrow \mathcal{F}(X)$ um operador acretivo e sci num espaço de Banach $X$, onde

$$
\mathcal{F}(X)=\{S \subset X: S \text { é não vazio, convexo e fechado }\} .
$$

Então, para cada $x_{0} \in X$ existe uma função $u:[0, \infty) \rightarrow X$ de classe $C^{1}$ satisfazendo

$$
\left\{\begin{array}{l}
\frac{d u}{d t}+A u \ni 0, t \geq 0 \\
u(0)=x_{0}
\end{array}\right.
$$

Prova: O teorema de E. Michael [43] garante a existência de uma seleção contínua $f: X \rightarrow$ $X$ para o operador $A$. Como $f$ é contínua e acretiva, existe uma única funçâo $u:[0, \infty) \rightarrow X$ de classe $C^{1}$, veja [3], satisfazendo

$$
\left\{\begin{array}{l}
\frac{d u}{d t}+f(u(t))=0 \\
u(0)=x_{0}
\end{array}\right.
$$

Isto conclui a prova.

Observação 3.10 Note que para X Gateaux suave, Au é sempre convexo e fechado para todo A acretivo e maximal. Veja [59].

Decorre de um resultado de Lakshmikantam [14] e de Fitzgibbon [23] que se $A$ é um operador acretivo sobre um espaço de Banach $X$ e que admite uma seleção $S: X \rightarrow X$ fraca-contínua satisfazendo à condição de Kamke então o problema $P A C(0 ; x), x \in X$, tem uma solução global forte.

O seguinte teorema generaliza um resultado de Fitzgibbon [23].

Teorema 3.11 Seja $A(t), t \geq 0$, uma familia de operadores acretivos (unívocos) fracocontínuos em $[0, \infty) \times X$. Suponha que para cada $t \geq 0$ existe um $\delta>0$ tal que se $0 \leq s \leq \delta$ então

$$
A(t) \subseteq A(t+s) .
$$

Sejam $x_{0} \in X$ e $b>0$. Suponha que exista $M \geq 0$ tal que

$$
\|A(t) x\| \leq M, \forall(t, x) \in[0, \infty) \times B\left(x_{0}, b\right),
$$

onde $B\left(x_{0}, b\right)$ é a bola de centro $x_{0}$ e raio $b$. Suponha que exista uma função de Kamke $g$ tal que

$$
\beta[A(t) U] \leq g(\beta(U)), \forall U \subset B\left(x_{0}, b\right) .
$$

Então, o problema $P A(t) C\left(0 ; x_{0}\right)$ tem uma soluçâo global forte e a família $A(t), t \geq 0$, é $m$-acretiva. 
Prova: Nestas condiçòes, conforme [14] e [23] existe uma solução local Lipschitz contínua $u$ que é também solução forte de $P A\left(t_{0}\right) C\left(0, x_{0}\right), \forall t_{0} \geq 0$. Seja $u$ solução de $P A\left(t_{0}\right) C\left(0, x_{0}\right)$ e suponha que esteja definida no intervalo máximo $\left[0, T_{x_{0}}\right)$. Afirmamos que $T_{x_{0}}=\infty$. Suponha, com vistas a um absurdo, que não, então dado $t \in\left[0, T_{x_{0}}\right)$ existe $\delta>0$ tal que se $0 \leq s<\delta,(t+s) \in\left[0, T_{x_{0}}\right)$ então,

$$
\frac{d}{d t}\|u(t+s)-u(t)\|^{2}=2\langle y(t+s)-y(t), u(t+s)-u(t)\rangle_{s}
$$

onde $y(t+s) \in A\left(t_{0}\right) u(t+s)$ e $y(t) \in A\left(t_{0}\right) u(t)$. Segue da acretividade de $A\left(t_{0}\right)$ que

$$
\frac{d}{d t}\|u(t+s)-u(t)\|^{2} \leq 0
$$

isto é,

$$
\|u(t+s)-u(t)\| \leq\|u(s)-u(0)\|=\left\|u(s)-x_{0}\right\| .
$$

Dado $\varepsilon>0$, da continuidade de $u$ existe $\delta_{1}$ tal que se $0 \leq s<\delta_{1}$, então

$$
\left\|u(s)-x_{0}\right\|<\varepsilon .
$$

Tomando $\delta_{0}=\min \left\{\delta, \delta_{1}\right\}$, então

$$
\|u(t+s)-u(t)\|<\varepsilon, 0 \leq s<\delta_{0} .
$$

Fazendo $t \rightarrow T_{x_{0}}$, obtemos que $\lim _{t \rightarrow T_{x_{0}}} u(t)=u\left(T_{x_{0}}\right)$ existe, contrariando a maximalidade de $T_{x_{0}}$. Logo, $T_{x_{0}}=\infty$ e $u$ é solução global de $P A\left(t_{0}\right) C\left(0 ; x_{0}\right)$.

Se $u_{0}$ é a solução global forte de $P A(0) C\left(0, x_{0}\right)$, segue da inclusão que $u_{0}$ também é solução de $P A\left(t_{0}\right) C\left(0, x_{0}\right), \forall t_{0} \geq 0$ e portanto solução forte de $P A(t) C\left(0, x_{0}\right)$.

Para a segunda afirmação, basta provar que para cada $t \geq 0, A(t)$ é $m$-acretivo. Mas isto está provado em Fitzgibbon [23].

Observação 3.12 Observe que a familia $A(t) \equiv A, t \geq 0$, com A acretivo sempre satisfaz à condição de inclusấo do teorema.

Enunciamos a seguir um resultado de existência inspirado nos trabalhos de Lakshmikantham, Fitzgibbon e no teorema acima.

Corolário 3.13 Sejam $A$ um operador acretivo com $D(A)=X$ e $S: X \rightarrow X$ uma seleção fraco-contínua. Suponha que $S$ satisfaz à condição de Kamke

$$
\beta(S(N)) \leq g(\beta(N)), \forall N \subset X \text { limitado. }
$$

Seja $x \in X$ e $F: C([0, T] ; X) \rightarrow B V([0, T] ; X)$ contínuo e limitado satisfazendo $\mathbf{H} 3$. Se $\mathbf{H} 4$ ou $\mathbf{H 5}$ ou $\mathrm{H} 6$ é satisfeita, então $P A C(F u ; x)$ tem uma soluçâo local Lipschitz contínua. 
Prova: Como $S$ é fraco-contínuo, a condição de Kamke implica que $S$ é $m$-acretivo (Teorema 3.11), e portanto $A$ é $m$ - acretivo. Agora o resultado segue do Teorema 3.5.

Note que o problema $P A C(F u ; x) \operatorname{com} F \not \equiv 0$ e $A$ fraco-contínuo sobre $X$ não se sabia ainda ter solução. Outra observação é que se $X$ é reflexivo e a seleção $S$ leva subconjuntos limitados em subconjuntos limitados, a condição de Kamke pode ser retirada pois ambos os lados da desigualdade são nulos.

Corolário 3.14 Seja $A(t), t \geq 0$, uma família de operadores acretivos satisfazendo a condiçâo de inclusão do Teorema 3.11. Suponha que $A(t)$ admite uma seleção $S(t): X \rightarrow X$ fracocontínua nas condiçôes do Teorema 3.11. Então:

1) $A(t), t \geq 0$ é $m$-acretiva e satisfaz $\mathbf{H 1}$ e $\mathbf{H 2}$.

2) Se F satisfaz $\mathbf{H 3}$ e, $\mathbf{H 4}$ ou $\mathbf{H 5}$ ou $\mathbf{H 6}$, então $P A(t) C(F u ; x)$ tem uma soluçâo local forte.

A demonstração decorre imediatamente do Teorema 3.11 e do Teorema 2.7.

Falkner [21] provou que $A: X \rightarrow X$ apenas fraco-contínuo não é suficiente para garantir a existência de solução para $\operatorname{PAC}(0 ; x)$, mesmo no sentido fraco. Hipóteses adicionais sobre o espaço $X$ ou sobre o operador $A$ são então necessárias.

E interessante notar que a existência de seleção $S$ para um operador acretivo $A$ sobre um espaço de Banach $X$ está muito próximo da noção de homeomorfismo. De fato, sabemos que se $S$ satisfaz

$$
(S x-S y, x-y)_{+} \geq c\|x-y\|^{2},
$$

com $c>0$, então $S$ é um homeomorfismo em X. Mais resultados nesta direção podem ser encontrados em Deimling [18]. Sobre existência de seleções veja [43] e [56].

Para o próximo resultado, utilizaremos o seguinte Teorema do Máximo, cuja demonstração pode ser encontrada em [20].

Teorema 3.15 (Teorema do Máximo) Sejam $X$ e $Y$ espaços topológicos (Hausdorff), $A: Y \rightarrow 2^{X}$ contínua com valores compactos $e W: X \times Y \rightarrow \mathbf{R}$ contínua. Então:

1) $Y \ni y \stackrel{V}{\mapsto} \sup _{y \in A(x)} W(x, y) \in \mathbf{R}$ é contínua.

2) $Y \ni y \stackrel{M}{\mapsto}\{x \in A(y) ; V(y)=W(x, y)\} \subset X$ é scs.

Observação 3.16 Segue da definição de semicontinuidade que se $M$ é univalente, então $M$ é contínua, sendo portanto uma seleção contínua para $A$.

Corolário 3.17 Sejam $X$ um espaço de Banach e $A: X \rightarrow 2^{X}$ um operador acretivo fracofraco-contínuo com valores fraco-compactos e $W: X \times X \rightarrow \mathbf{R}$. Suponha que $W$ determina uma função (unívoca) $M$ como no Teorema 3.15 e que

$$
\beta(M(N)) \leq g(\beta(N)), \forall N \text { limitado. }
$$

Então, o problema PAC $\left(0, x_{0}\right)$ tem uma solução local Lipschitz. Além disso, A ém-acretivo.

Prova: Como $M$ é uma seleção fraco-contínua para $A$ e satisfaz as hipóteses do Corolário 3.13 , o resultado segue imediatamente. 
Em [47] prova-se que se $x \in \hat{D}(A(0))$ e $h$ da hipótese $\mathbf{H 1}$ é de variação limitada em $[0, T]$, então a função

$$
t \mapsto U(t, 0) x=u(t)
$$

é Lipschitz contínua em $[0, T]$, onde

$$
U=\{U(t, s): 0 \leq s \leq t \leq T\}
$$

é o operador de evolução associado à família de operadores $A(t), 0 \leq t \leq T$. Também $u$ é solução integral de $P A(t) C(0 ; x)$ em $[0, T]$. Veja [47]

O próximo resultado melhora o Teorema 3.5 .

Teorema 3.18 Seja $A(t), 0 \leq t \leq T$, uma familia de operadores $m$-acretivos satisfazendo H1 e H2. Seja F : $C([0, T] ; X) \rightarrow C([0, T] ; X) \cap B V([0, T] ; X)$ contínuo e limitado. Suponha que $B_{x} F$ satisfaça $\mathbf{H 4}, \mathbf{H} 5$ ou $\mathbf{H 6}$ e que em $\mathbf{H 1}$ h seja de variação limitada em $[0, T]$. Entâo, dado $x \in \hat{D}(A(0))$, o problema $P A(t) C(F u ; x)$ tem uma solução local Lipschitz contínua.

Prova: Pelo Teorema 2.7 do Capítulo 2 dado $\varepsilon>0$ existe um $\delta>0$ tal que

$$
\left(T_{\delta} B_{x} F E_{\delta}\right)\left(C_{\varepsilon}^{\delta}\right) \subset C_{\varepsilon}^{\delta} .
$$

Segue das hipóteses que o conjunto dos pontos fixos $I(F)$ deste operador é não vazio. Como o operador de Bénilan-Pavel leva funções de $B V \cap C([0, T] ; X)$ em funções Lipschitz contínuas, então o problema abstrato de Cauchy $P A(t) C(F u ; x)$ tem pelo menos uma solução local Lipschitz contínua em $[0, \delta]$.

Corolário 3.19 Se $x \in D(A(0)), h$ da hipótese $\mathbf{H 1}$ é de variação limitada em $[0, T]$ e $X$ tem RNP, então a soluçấo local de $P A(t) C(F u ; x)$ é uma solução forte.

Prova: Como a solução local é de variação limitada e $X$ tem RNP, então a solução é forte. $\square$

Exemplo 3.20 (Equação da onda não linear) Seja $\Omega \subset \mathbf{R}^{N}$ um aberto limitado com fronteira suave $\Gamma$ e consideremos o problema

$$
\left\{\begin{array}{l}
\frac{\partial^{2} u}{\partial t^{2}}-\Delta u+\beta\left(\frac{\partial u}{\partial t}\right) \ni(f u)(t, x) \text { em } \mathbf{R}^{+} \times \Omega \\
u=0 \text { em } \partial \Omega \\
u(0, x)=u_{0}(x) \text { em } \Omega \\
\frac{\partial u}{\partial t}(0, x)=v_{0}(x) \text { em } \Omega
\end{array}\right.
$$

Fisicamente a equação descreve as vibraçôes de uma membrana elástica com fronteiras fixas e sujeita a uma força de atrito $\beta$ e força externa $f$.

Suponha que:

a)f:C([0,T]; $\left.L^{2}(\Omega)\right) \rightarrow C\left([0, T] ; L^{2}(\Omega)\right)$ satisfaz

$$
|f u(t)-f v(t)|_{L^{2}(\Omega)} \leq c t\|u-v\|_{\infty}
$$


para quase todo $t \in[0, T]$,

b) $\beta$ é operador monótono maximal $\operatorname{com} \beta(0) \ni 0$

Como $\beta$ é monótono maximal e $\beta(0) \ni 0$ existe $j: \mathbf{R} \rightarrow(-\infty, \infty]$ tal que $\partial j=\beta$. Considere $\varphi: H_{0}^{1}(\Omega) \rightarrow(-\infty, \infty]$ dada por

$$
\varphi(v)=\left\{\begin{array}{l}
\int_{\Omega} j(v(x)) d x, \text { se jov } \in L^{1}(\Omega) \\
+\infty, \text { caso contrário. }
\end{array}\right.
$$

Claramente $\varphi$ é própria, convexa e semicontínua inferior e sua extensão a $L^{2}(\Omega)$ também. Seja então $\tilde{\beta}=\partial \varphi$, definida em $D(\tilde{\beta}) \subset H_{0}^{1}(\Omega)$, portanto $\tilde{\beta}$ é monótono maximal. Sabemos que $-\Delta: H_{0}^{1}(\Omega) \rightarrow H^{-1}(\Omega)$ também é monótono maximal. Lembramos que os mergulhos

$$
H_{0}^{1}(\Omega) \mapsto L^{2}(\Omega) \mapsto H^{-1}(\Omega)
$$

sâo compactos.

Fixado $t \geq 0, u(t)$ denotará a funçâo $x \mapsto u(t, x)$. Fazendo $v=\frac{\partial u}{\partial t}, U=\left[\begin{array}{l}u \\ v\end{array}\right], U_{0}=\left[\begin{array}{l}u_{0} \\ v_{0}\end{array}\right]$ e $A=\left[\begin{array}{cc}0 & -I \\ -\Delta & \tilde{\beta}\end{array}\right]$, o problema acima pode ser escrito na forma

$$
\left\{\begin{array}{l}
\frac{d U}{d t}+A U \ni F(U)(t), t \geq 0 \\
U(0)=U_{0}
\end{array}\right.
$$

onde $\left.U \in C\left([0, T], H_{0}^{1} \Omega\right) \times L^{2}(\Omega)\right), F: C\left([0, T] ; H_{0}^{1}(\Omega) \times L^{2}(\Omega)\right) \rightarrow C\left([0, T] ; H_{0}^{1}(\Omega) \times L^{2}(\Omega)\right)$ com $F U=F(u, v)=(0, f u)$ e o espaço de Banach é $X=H_{0}^{1}(\Omega) \times L^{2}(\Omega)$ munido do produto interno

$$
\left(U_{1}, U_{2}\right)=\int_{\Omega} \nabla u_{1} \cdot \nabla u_{2} d x+\int_{\Omega} v_{1} v_{2} d x
$$

onde $U_{i}=\left[\begin{array}{l}u_{i} \\ v_{i}\end{array}\right]$

O operador A e seu domínio são

$$
\begin{gathered}
D(A)=\left\{(p, q) \in\left(H_{0}^{1}(\Omega) \times H_{0}^{1}(\Omega)\right):(-\Delta p+\tilde{\beta}(q)) \cap L^{2}(\Omega) \neq \emptyset\right\} \subset X, \\
A(p, q)=\{-q\} \times(-\Delta p+\tilde{\beta}(q)) \cap L^{2}(\Omega) \subset X,
\end{gathered}
$$

para todo $(p, q) \in D(A)$. Sabemos de [3] que A é monótono maximal e que $\overline{D(A)}$ é dado por

$$
\overline{D(A)}=H_{0}^{1}(\Omega) \times\left\{\xi \in L^{2}(\Omega): \xi(x) \in \overline{D(j)} \text { a.e. } x \in \Omega\right\}
$$

Como F satisfaz

$$
\left|F U_{1}(t)-F U_{2}(t)\right| \leq c t\left\|u_{1}-u_{2}\right\|_{\infty},
$$

onde $U_{i}=\left(u_{i}, v_{i}\right)$, segue do Corolário 2.16 ou do Teorema 2.7 que o problema (*) acima tem uma soluçâo local integral. Observamos que se $F U$ é de variação limitada e se $U_{0} \in \hat{D}(A)$. entâo do Teorema 3.4 que o problema $\left(^{*}\right)$ tem uma soluçâo local Lipschitz contínua. 
De fato neste exemplo basta supor $F U \in L^{1}(0, T ; X)$ para garantirmos a existência de solução integral, pois o operador $A$ independe de $t$. Um importante caso particular da equaçào da onda nào linear é

$$
\begin{aligned}
& \frac{\partial^{2} u}{\partial t^{2}}-\frac{\partial^{2} u}{\partial x^{2}}+\mu \operatorname{sign}\left(\frac{\partial u}{\partial t}\right) \ni f(t, u), t>0, x \in[0,1] \\
& u(t, 0)=u(t, 1)=0, \quad t>0
\end{aligned}
$$

que descreve as vibrações de uma corda de comprimento 1, com as extremidades fixas numa superfície sólida plana que opõe ao movimento da corda uma força de atrito de magnitude $\operatorname{sign}\left(\frac{\partial u}{\partial t}\right)$. Veja [46] e [3]. 


\section{Capítulo 4}

\section{Problemas Normais}

Neste capítulo daremos uma aplicação do Princípio Variacional de Ekeland às equaçòes de evolução em espaços de Hilbert. Estudaremos algumas propriedades do operador de BénilanPavel e provaremos um resultado de existência de solução para problemas normais.

\subsection{Introdução}

Antes de enunciarmos os resultados vamos precisar de alguns conceitos.

Definição 4.1 Sejam $X$ um espaço topológico Hausdorff e $f: X \rightarrow \mathbf{R} \cup\{\infty\}$.

1) $f$ é semicontínuo inferiormente (sci) se para cada $a \in \mathbf{R}$, o conjunto

$$
\{x \in \dot{X}: f(x)>a\} \text { é aberto. }
$$

2) $f$ é sequencialmente semicontínuo inferiormente em $x_{0} \in X$ se para qualquer sequência $\left(x_{n}\right)$ convergente para $x_{0}$ vale

$$
f\left(x_{0}\right) \leq \liminf f\left(x_{n}\right) .
$$

Dizemos que $f$ é sequencialmente semicontinuo inferiormente em $X$ se $f$ é sequencialmente semicontínuo inferiormente em $x \in X$ para todo $x \in X$.

Observação 4.2 A relação entre estes dois conceitos pode ser resumida no seguinte:

- 1) implica 2),

- 2) implica 1) quando X satisfaz ao primeiro axioma de enumerabilidade.

Segue que num espaço de Banach $X$ os dois conceitos coincidem.

Observação 4.3 Num espaço de Banach X podemos definir semicontinuidade e semicontinuidade sequencial tanto na topologia forte como na topologia fraca. Como sequências convergentes de $X$ são fracamente convergentes, segue que funcionais sequencialmente fracamente sci são sequencialmente semicontínuos inferiormente. Por outro lado, como todo aberto da topologia fraca contém um aberto da topologia forte, temos que todo funcional semicontínuo fraco inferiormente é semicontínuo inferiormente. Sob algumas condiçôes as duas 
reciprocas são verdadeiras, por exemplo, se o funcional for convexo ou se $X$ for reflexivo ou mais geralmente, se a topologia fraca satisfizer o primeiro axioma de enumerabilidade.

Como a topologia fraca de um espaço de Banach $X$ não satisfaz o primeiro axioma de enumerabilidade, não podemos garantir que semicontinuidade sequencial inferior na topologia fraca implique semicontinuidade inferior na mesma topologia. Garantimos apenas que se $A \subset$ $X$ é limitado e $X$ reflexivo, todo funcional sequencialmente fraco semicontínuo inferiormente em A é fraco semicontinuo inferiormente em $A$. Veja [22] para mais detalhes.

Observação 4.4 Lembramos que um operador $F: X \rightarrow Y$ (não necessariamente linear) entre espaços de Banach é compacto se é contínuo e leva subconjuntos limitados de $X$ em subconjuntos relativamente compactos de $Y$. F é completamente contínuo se leva sequências fracamente convergentes em sequências fortemente convergentes.

Em 1972 Ivar Ekeland demonstrou o hoje conhecido Princípio Variacional de Ekeland para espaços métricos completos $X$. A partir dele Caristi provou um teorema de ponto fixo para aplicações com valores nas partes de $X$, que mais tarde constatou-se ser equivalente ao Princípio Variacional de Ekeland. Veja [44].

Vejamos seus enunciados.

\section{Teorema 4.5 (Princípio Variacional de Ekeland)}

Sejam $(M, d)$ um espaço métrico completo e $f: M \rightarrow \mathbf{R} \cup\{\infty\}$ um funcional sci e limitado inferiormente. Dados $\varepsilon>0$ e $\bar{u}$ tais que

$$
f(\bar{u}) \leq \inf _{x \in M} f(x)+\frac{\varepsilon}{2}
$$

então para qualquer $\lambda>0$ existe $u_{\lambda} \in M$ tal que:

1) $f\left(u_{\lambda}\right) \leq f(\bar{u})$

2) $d\left(u_{\lambda}, \bar{u}\right) \leq \lambda$

3) $f\left(u_{\lambda}\right)<f(u)+\frac{\varepsilon}{\lambda} d\left(u, u_{\lambda}\right), \forall u \neq u_{\lambda}$.

Teorema 4.6 (Ponto Fixo de Caristi)

Sejam $(M, d)$ um espaço métrico completo e $g: M \rightarrow \mathbf{R} \cup\{\infty\}$ semicontínua inferiormente, própria e limitada inferiormente. Se $T: M \rightarrow 2^{M}$ tem a propriedade

$$
d(x, y)+g(y) \leq g(x)
$$

para cada $x \in M$ e $y \in T x$, então existe $z \in M$ tal que $z \in T z$.

A prova destes resultados pode ser encontrada em [22] e em [38]. Para uma generalização dos mesmos veja [44].

\subsection{Aplicações}

A) Consideremos o seguinte problema:

$$
\left\{\begin{array}{l}
\frac{d u}{d t}+B(t) u(t)=f(t), 0<t<T, \\
u(0)=x
\end{array}\right.
$$


onde $B(t)$ é um operador contínuo sobre o espaço de Hilbert $H$ e $f \in C([0, T] ; H)$.

Suponha que $B(t)$ admita uma decomposição do tipo

$$
B(t)=A(t)+A_{1}(t), \quad 0 \leq t \leq T,
$$

com $A(t)$ linear autoadjunto, contínuo e monótono maximal. Definimos:

$A_{1}: C([0, T] ; H) \rightarrow C([0, T] ; H)$,

$F: C([0, T] ; H) \rightarrow C([0, T] ; H)$,

$g(t, \cdot): H \rightarrow \mathbf{R}$, dadas por :

- $\left(A_{1} u\right)(t)=A_{1}(t) u(t)$,

- $(F u)(t)=f(t)-\left(A_{1} u\right)(t)$,

- $g(t, x)=\frac{1}{2}\langle x, A(t) x\rangle$.

Assim o problema $(P 1)$ pode ser reescrito na forma de um problema abstrato:

$$
\left\{\begin{array}{l}
\frac{d u}{d t}+\partial g(t, u(t)) \ni(F u)(t), 0<t<T, \\
u(0)=x .
\end{array}\right.
$$

Se a parte não monótona de $B(t)$, isto é, $A_{1}(t)$, tem algumas propriedades tais como " $B_{x} F$ compacto" e "F leva subconjuntos limitados em subconjuntos uniformemente integráveis" e a parte monótona satisfaz às hipótese $\mathbf{H 1}$ e $\mathbf{H 2}$ do Capítulo 2, então o Teorema 2.7 do Capítulo 2 pode ser aplicado para provar a existência de uma solução integral local do problema $(P 1)$.

B) Outro exemplo é o clássico problema de valor inicial. Consideremos o problema de determinar uma soluçào de

$$
\left\{\begin{array}{l}
\frac{d u}{d t}=f(t, u(t)), 0<t<T \\
u(0)=x \in \mathbf{R}^{n}
\end{array}\right.
$$

onde $f:[0, T] \times \mathbf{R}^{n} \rightarrow \mathbf{R}^{n}$ é contínua.

Escolhemos $A:[0, T] \rightarrow L\left(\mathbf{R}^{n}\right)$ uma aplicação linear, contínua com $A(t)$ autoadjunto, monótono maximal e definimos $g:[0, T] \times \mathbf{R}^{n} \rightarrow \mathbf{R}$ por

$$
g(t, x)=\frac{1}{2}\langle x, A(t) x\rangle .
$$

Como $\partial g(t, x)=A(t) x$, o problema $(P 3)$ pode ser reescrito na forma:

$$
\left\{\begin{array}{l}
\frac{d u}{d t}+A(t) u(t) \ni(F u)(t), \\
u(0)=x
\end{array}\right.
$$

onde $F(u)(t)=f(t, u(t))+A(t) u(t)$. 
Podemos escolher a família $A(t)$ satisfazendo $\mathbf{H 1}$ e $\mathbf{H 2}$. Se além disso $F$ satisfaz às hipóteses $\mathbf{H 3}$ e $\mathbf{H 4}$ ou $\mathbf{H 5}$ ou $\mathbf{H 6}$ então o Teorema 2.7 pode ser usado para provar a existência de solução local integral.

C) Consideremos o seguinte problema

$$
\left\{\begin{array}{l}
\frac{d u}{d t}+A(t) u(t)=f(t), 0<t<T \\
u(0)=u_{0},
\end{array}\right.
$$

onde para cada $t \in[0, T], A(t)$ é um operador linear contínuo em $H$ e $f \in L^{2}([0, T] ; H)=Y$.

Suponha $A(t)=A_{1}(t)+B(t)$, com $A_{1}(t)$ linear contínuo, autoadjunta e positiva definida. Tomando

$$
H \ni x \stackrel{f_{1}(t, \cdot)}{\mapsto} \frac{1}{2}\left\langle x, A_{1}(t) x\right\rangle \in \mathbf{R},
$$

e $F(t, u)=f(t)-B(t) u(t)$, o problema $(P 5)$ acima pode ser reescrito como

$$
\left\{\begin{array}{l}
\frac{d u}{d t}+\partial f_{1}(t, u(t)) \ni F(t, u(t)) \\
u(0)=u_{0} .
\end{array}\right.
$$

Suponha que as aplicações

$(A u)(t)=A(t) u(t)$

$\left(A_{1} u\right)(t)=A_{1}(t) u(t)$,

$(B u)(t)=B(t) u(t)$

definidas em Y sejam lineares contínuas e que existe $c>0$ tal que

$$
\left[u, A_{1} u\right] \geq c[u, u]
$$

para todo $u \in Y$. O símbolo $[\cdot, \cdot]$ denota o produto interno de $Y$.

Sabemos de [2] que existe um funcional $J$ associado a problemas do tipo $(P 6)$ tal que $J(u)=0 \Longleftrightarrow u$ é solução de $(P 6)$. Sejam $V$ e $K$ dados respectivamente por $V=\{v \in Y$ : $\dot{v} \in Y\}$ e $K=\left\{v \in V: v(0)=u_{0}\right\}$, então o funcional $J$ associado ao problema $(P 6)$ é dado por:

$$
J(v)=\frac{1}{2} \int_{0}^{T} f_{1}\left(t, v(t)-A_{1}^{-1}(t)(f(t)-B(t) v(t)-\dot{v}(t)) d t\right.
$$

e é não negativo, próprio, convexo e fraco-semicontínuo inferiormente em $V$ e portanto em $K$. Note que $K$ é convexo e fechado em $V$ e que $v \in V$ implica que $v:[0, T] \rightarrow H$ é contínua. Note que $V$ é um espaço de Hilbert sob a norma

$$
\|v\|_{V}^{2}=\int_{0}^{T}\left[\|v(t)\|_{Y}^{2}+\|\dot{v}(t)\|_{Y^{*}}^{2}\right] d t
$$

Segue da convexidade que $J$ é semicontínuo inferiormente e portanto contínuo no interior do seu domínio efetivo. Disto podemos extrair algumas informações, a mais imediata é que $0 \in \partial J(\bar{x})$ se, e somente se, $\bar{x}$ minimiza $J$. Do Teorema de Bronsted-Rockafellar, para $\bar{x} \in \operatorname{dom}(J)$, existe uma sequência $\left(x_{k}\right)$ tal que 
a) $x_{k} \rightarrow \bar{x}$

b) $J\left(x_{k}\right) \rightarrow J(\bar{x})$

c) $\partial J\left(x_{k}\right) \neq \emptyset, \forall k$.

Também do Princípio Variacional de Ekeland existe uma aplicação $G$ Lipschitz contínua e $\hat{v} \in V$ tal que $\hat{v}$ seja mínimo estrito de $(J+G)$.

Do Teorema 4.5 podemos provar que para uma função $f$ semicontínua inferiormente, Gateaux diferenciável e limitada inferiormente, existe uma sequência minimizante particular $\left(y_{n}\right)$ tal que $f\left(y_{n}\right) \rightarrow \inf f$ e $f^{\prime}\left(y_{n}\right) \rightarrow 0 \mathrm{em} X^{*}$. Não é verdade, em geral, que existe $\bar{y}$ pertencente ao fecho do conjunto $\left\{y_{n}: n\right.$ é natural $\}$, satisfazendo $f^{\prime}(\bar{y})=0$. Isso sugere a seguinte definição.

Definição 4.7 Seja $X$ um espaço de Banach.

1) Se $f: X \rightarrow \mathbf{R}$ é um funcional Gateaux diferenciável, dizemos que $f$ satisfaz à condição $(C)$ de Palais-Smale sobre um subespaço $V \subset X$ se existe uma sequência $\left(y_{n}\right)$ em $V$ com

$$
\begin{gathered}
\left|f\left(y_{n}\right)\right| \leq \text { constante }, \\
f^{\prime}\left(y_{n}\right) \rightarrow 0 \text { em } X^{*}
\end{gathered}
$$

então no fecho do conjunto $\left\{y_{n}: n\right.$ é natural $\}$ existe algum ponto $\bar{y}$ onde $f^{\prime}(\bar{y})=0$.

2) O funcional $f$ satisfaz à condição fraca $(C)$ sobre o subespaço $V \subset X$ se existe uma sequência $\left(y_{n}\right)$ em $V$ com:

1i) $\left|f\left(y_{n}\right)\right| \leq$ constante

2i) $f^{\prime}\left(y_{n}\right) \neq 0, \forall n$

3i) $f^{\prime}\left(y_{n}\right) \rightarrow 0 \mathrm{em} X^{*}$,

então existe algum $\bar{y} \in X$ tal que $\liminf f\left(y_{n}\right) \leq f(\bar{y}) \leq \limsup f\left(y_{n}\right)$ e $f^{\prime}(\bar{y})=0$.

Sabemos que se $f$ satisfaz à condição $(C)$ de Palais-Smale, então $f$ satisfaz à condição fraca $(C)$. Veja $[20]$.

O próximo resultado é um caso particular de um resultado mais geral (e já conhecido) em espaços reflexivos. Veja também [20]. Voltando ao problema $(P 6)$ temos a seguinte proposição:

Proposição 4.8 Suponha J Gateaux diferenciável e que

$$
\frac{1}{2}[v,(A+B) v] \geq c\|v\|_{Y}^{2}, \forall v \in V .
$$

Então, J satisfaz à condição fraca $(C)$ em $V$.

Prova: A hipótese implica que $J$ satisfaz

$$
J(v) \geq c\|v\|_{Y}^{2}-\|v\|_{Y}\|f\|_{Y^{*}}-\frac{1}{2}\left\langle u_{0}, u_{0}\right\rangle
$$

e portanto $J(v) \rightarrow \infty$ quando $\|v\|_{Y} \rightarrow \infty$. 
Dada uma sequência $\left(v_{n}\right)$ nas condições da Definição $4.7(2)$, existe uma subsequência $\left(u_{n}\right)$ tal que

$$
\lim J\left(u_{n}\right)=\lim \sup J\left(v_{n}\right) .
$$

Como $V$ é reflexivo e $J$ é coercivo existe uma subsequência $\left(w_{n}\right)$ de $\left(u_{n}\right)$ que converge fracamente para algum $\bar{v}$. Como $J$ é fraco-semicontínuo inferiormente, obtemos

$$
J(\bar{v}) \leq \lim J\left(w_{n}\right)=\limsup J\left(v_{n}\right) .
$$

Como $J$ é convexo e Gateaux diferenciável,

$$
\left\langle J^{\prime}\left(w_{n}\right), v-w_{n}\right\rangle+J\left(w_{n}\right) \leq J(v), \forall v \in Y .
$$

Da condição $3 i$ ) obtemos

$$
\limsup J\left(v_{n}\right)=\lim J\left(w_{n}\right) \leq J(v) .
$$

Como $v$ é arbitrário, segue que $\bar{v}$ minimiza $J$ e que $J^{\prime}(\bar{v})=0$. Então,

$$
\limsup J\left(v_{n}\right)=\inf J,
$$

$\operatorname{assim} J\left(v_{n}\right)$ converge para $J(\bar{v})$.

Não podemos garantir que $J(\bar{v})=0$. Se isto ocorrer, então $\bar{v}$ seria solução de $(P 6)$. Observamos que $J$ é coercivo em $K$ e portanto $J$ assume seu ínfimo em $K$.

Com uma hipótese adicional sobre sequências minimizantes podemos melhorar o resultado da proposição anterior e obter existência de solução para o problema $(P 6)$.

Definição 4.9 Um funcional $F: X \rightarrow \mathbf{R}$ localmente Lipschitziano satisfaz a condição de Chang se para toda sequência $\left(u_{n}\right)$ de $X$ tal que

1i) $F\left(u_{n}\right) \rightarrow c=\inf _{X} F$

2i) $m\left(u_{n}\right)=\min \left\{\left\|\mu_{n}\right\|_{*} ; \mu_{n} \in \partial F\left(u_{n}\right)\right\} \rightarrow 0$, então $\left(u_{n}\right)$ possui uma subsequência convergente.

Quando o espaço $X$ é reflexivo, sabemos de [20] que a condição de Chang é equivalente à seguinte condição $[P S]_{c}$ :

se $\left(u_{n}\right)$ é uma sequência em $X$ e $\left(\varepsilon_{n}\right),\left(\delta_{n}\right) \in \mathbf{R}^{+}$são sequências reais convergentes para zero, tais que

1i) $F\left(u_{n}\right) \rightarrow c=\inf _{X} F$

2i) $F\left(u_{n}\right) \leq F(u)+\varepsilon_{n}\left\|u_{n}-u\right\|$, se $\left\|u_{n}-u\right\| \leq \delta_{n}$, então $\left(u_{n}\right)$ possui uma subsequência convergente.

Proposição 4.10 Seja J o funcional associado ao problema $(P 6)$ e suponha que $J$ satisfaz $[P S]_{0} e \inf _{K} J=0$. Então o problema tem pelo menos uma soluçấo.

Prova: Como $V$ é reflexivo, $J$ é semicontínuo inferiormente e convexo, então $J$ é contínuo no interior do seu domínio efetivo. Além disso, $F$ é localmente Lipschitziano.

Seja $\left(v_{n}\right)$ uma sequência minimizante em $K \operatorname{com} \delta_{n}=J\left(v_{n}\right)>0, \delta_{n} \rightarrow 0$. Para cada inteiro positivo $n$, tomamos $\varepsilon=\delta_{n}, \lambda=\delta_{n}^{\frac{1}{2}}$ no Princípio Variacional de Ekeland e obtemos 
uma sequência $\left(u_{n}\right)$ em $K$ tal que

1i) $J\left(u_{n}\right) \leq J\left(v_{n}\right)$

2i) $\left\|u_{n}-v_{n}\right\| \leq \sqrt{\delta_{n}}$,

3i) $J\left(u_{n}\right)<J(u)+\sqrt{\delta_{n}}\left\|u-u_{n}\right\|$ se $u \neq u_{n}$.

Assim $J\left(u_{n}\right) \rightarrow 0$ e $J\left(u_{n}\right) \leq J(u)+\sqrt{\delta_{n}}\left\|u_{n}-u\right\|$, para todo $u \in K$ e portanto, satisfaz a condição de Chang. Segue então que $\left(u_{n}\right)$ possui uma subsequência convergente, ainda denotada por $\left(u_{n}\right)$, digamos $u_{n} \rightarrow w$. Logo, $J(w) \leq \liminf J\left(u_{n}\right)=0$, e portanto $J(w)=0$. Isto conclui a prova. $\square$

\subsection{Problemas normais}

Nesta seção fixamos $T=1$ e $A(t), 0 \leq t \leq 1$, uma família de operadores $m$-acretivos satisfazendo às condições $\mathbf{H 1}$ e $\mathbf{H 2}$ do Capítulo 2, $x \in D=\overline{D(A(0))}$ e consideramos o operador $B_{x}$ de Bénilan-Pavel.

Se existe uma aplicaçâo $F$ de $C([0,1] ; X)$ nas partes não vazias de $C([0,1] ; X)$ tal que $u \in\left(B_{x} F\right) v$ para algum $v \in C([0,1] ; X)$, então necessariamente existe uma função $f \in F(v)$ tal que $B_{x} f=u$. Tomando $A v=\left(B_{x} F\right) v-u, v \in C([0,1] ; X)$, recaímos no problema $A v \ni 0$, que é básico em Análise não linear. A existência de $f$ implica que $A v \ni 0$ admite uma soluçào em $C([0,1] ; X)$.

Nesta secção estudamos algumas propriedades de $\left(B_{x} F\right)$.

Definição 4.11 Se $X$ e $Y$ são espaços normados, uma aplicação $F: X \rightarrow \mathcal{P}(Y)$ é nâo expansiva se existe uma constante $0 \leq k \leq 1$ satisfazendo

$$
H(F u, F v) \leq k\|u-v\|_{X}
$$

onde para $A$ e $B \in \mathcal{P}(Y)$ tem-se

$$
H(A, B)=\max \left\{\sup _{a \in A} d(a, B), \sup _{b \in B} d(A, b)\right\},
$$

é a conhecida distância de Hausdorff entre $A$ e $B$. Quando $k<1, F$ é chamada uma contração.

Como usual denotamos por $C B(D)$ o espaço de todos os subconjuntos limitados e fechados de $D$ munido da métrica de Hausdorff $H$. Fixamos também $F: C([0,1] ; X) \rightarrow$ $\mathcal{P}(C([0,1] ; X))$.

É fácil ver que $B_{x}$ é um operador não-expansivo de $C([0,1] ; X)$ em $C([0,1] ; X)$. De fato, se $f$ e $g$ pertencem a $C([0,1] ; X)$, então

$$
\left\|B_{x} f(t)-B_{x} g(t)\right\| \leq \int_{0}^{t}\|f(s)-g(s)\| d s \leq\|f-g\|_{\infty} .
$$

Tomando o supremo em $t \in[0,1]$, obtemos

$$
\left\|B_{x} f-B_{x} g\right\|_{\infty} \leq\|f-g\|_{\infty} .
$$


Lema 4.12 Sejam $A(t), 0 \leq t \leq 1$, uma família de operadores $m$-acretivos em $X$ satisfazendo $\mathbf{H 1}$ e $\mathbf{H 2}$ e $F$ uma aplicação não expansiva de $C([0,1] ; X)$ nas partes nâo vazias de $C([0,1] ; X)$. Entâo, $\left(B_{x} F\right): C([0,1] ; X) \rightarrow \mathcal{P}(C([0,1] ; X))$ é também nâo expansiva.

Prova: Tomemos $u$ e $v$ em $C([0,1] ; X)$. Sejam $a \in\left(B_{x} F\right) u$ e $b \in\left(B_{x} F\right) v$, então existem

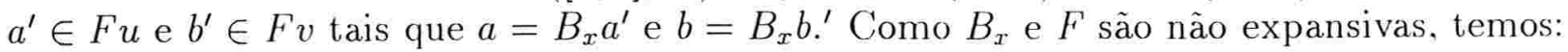

$$
\begin{gathered}
d\left(a, B_{x} F v\right)=d\left(B_{x} a^{\prime}, B_{x} F v\right)=\inf _{b^{\prime} \in F v} d\left(B_{x} a^{\prime}, B_{x} b^{\prime}\right) \leq \inf _{b^{\prime} \in F v} d\left(a^{\prime}, b^{\prime}\right)= \\
=d\left(a^{\prime}, F v\right) \leq \sup _{a^{\prime} \in F u} d\left(a^{\prime}, F v\right) \leq \max \left\{\sup _{a^{\prime} \in F u} d\left(a^{\prime}, F v\right), \sup _{b^{\prime} \in F v} d\left(F u, b^{\prime}\right)\right\}= \\
=H(F u, F v) \leq k\|u-v\|_{\infty} .
\end{gathered}
$$

Analogamente, $d\left(B_{x} F u, b\right) \leq k\|u-v\|_{\infty}$, e segue que

$$
H\left(B_{x} F u, B_{x} F v\right) \leq k\|u-v\|_{\infty},
$$

mostrando que $\left(B_{x} F\right)$ é não expansiva.

Notemos que a desigualdade acima mostra que $\left(B_{x} F\right)$ é Lipschitz contínua e portanto é semicontínua superiormente. Se $c o(K)$ denota a envoltória convexa de $K$, a aplicação $\left(\widetilde{B_{x} F}\right)$ definida por

$$
\left(\widetilde{B_{x} F}\right)(u)=\operatorname{co}\left(B_{x} F u\right)
$$

é também Lipschitz contínua com mesma constante $k$.

Lema 4.13 Seja $F$ uma aplicação de $C([0,1] ; X)$ nas partes não vazias de $C([0,1] ; X)$ que aplica subconjuntos limitados em conjuntos uniformemente integráveis. Seja $A(t), 0 \leq t \leq 1$, uma famítia de operadores $m$-acretivos satisfazendo $\mathbf{H 1}, \mathbf{H 2}$ e $\mathbf{H} 7$ em $X$. Entâo, existe $\delta>0$ tal que o conjunto é $T_{\delta} B_{x}\left(F E_{\delta} u\right)$ é relativamente compacto para todo $u \in C([0, \delta] ; X)$.

Prova: Segue do Teorema 2.7 do Capítulo 2 que dado $\varepsilon>0$ existe $\delta>0$ tal que se $v \in C_{\varepsilon}^{\delta}$ então para cada $f \in F E_{\delta} v$ temos,

$$
\left\|T_{\delta} B_{x} f(t)-T_{\delta} u_{0}(t)\right\| \leq \int_{0}^{\delta}\|f(s)\| d s \leq \varepsilon .
$$

Logo, $T_{\delta} B_{x} f \in C_{\varepsilon}^{\delta}, \forall f \in F E_{\delta} v$ e para todo $v \in C_{\varepsilon}^{\delta}$. Segue que $T_{\delta} B_{x} F E_{\delta}\left(C_{\varepsilon}^{\delta}\right) \subset C_{\varepsilon}^{\delta}$. Dado $u \in C([0,1] ; X)$ tomando $N=\{f: f \in F u\}$ como em H3 e usando o Corolário 2.21 obtemos que $T_{\delta} B_{x} F E_{\delta}(u)$ é relativamente compacto em $C([0, \delta] ; X)$.

Definição 4.14 Seja $D \subset X$ um subconjunto de um espaço normado $X$. Uma sequência $\left(F_{n}\right)$ de aplicaçôes de $D$ nas partes de $X$ é chamada assintoticamente regular se para cada $x_{1} \in D$ existe uma sequência $\left(x_{n}\right)$ em $X$ satisfazendo:

1) $x_{n+1} \in F_{n} x_{n}, n \geq 1$,

2) $\left\|x_{n+1}-x_{n}\right\| \rightarrow 0$ quando $n \rightarrow \infty$.

Uma aplicação $F$ é assintoticamente regular se a sequência constante $F_{n}=F$ é assintoticamente regular. 
Proposição 4.15 Sejam $A(t), 0 \leq t \leq 1$, uma família de operadores m-acretivos em $X$ satisfazendo $\mathbf{H 1}, \mathbf{H 2}$ e $\mathbf{H} 7$ e $F$ uma aplicaçâo nâo expansiva de $C([0,1] ; X)$ nas partes nâo vazias de $C([0,1] ; X)$ com valores compactos. Então, dado $\varepsilon>0$ existem $\delta>0, C_{\varepsilon}^{\delta}$ convexo e limitado tal que para qualquer sequência de reais $\left(t_{n}\right)$ com $0 \leq t_{n} \leq t<1$ e $\sum t_{n}=\infty$, a sequência

$$
P_{n}=\left(1-t_{n}\right) I+t_{n}\left(T_{\delta} B_{x} F E_{\delta}\right): C_{\varepsilon}^{\delta} \rightarrow C B\left(C_{\varepsilon}^{\delta}\right)
$$

é assintoticamente regular.

Prova: Notemos que $F$ leva subconjuntos limitados em subconjuntos uniformemente integráveis. Se $D=C_{\varepsilon}^{\delta}$, como $T_{\delta} B_{x} F E_{\delta}$ é não expansiva de $D$ com imagem em $C B(D)$ e $T_{\delta} B_{x} F E_{\delta} u$ é compacto por 4.13 , então a afirmação segue imediatamente de um resultado de D. Khang [33] que está no Capítulo 1 .

Note que $T_{\delta} B_{x} F E_{\delta}$ e $\left(P_{n}\right)$ têm o mesmo conjunto de pontos fixos e a vantagem é que $\left(P_{n}\right)$ é assintoticamente regular.

Proposição 4.16 Seja $F$ de $C([0,1] ; X)$ nas partes de $C([0,1] ; X)$ uma contração com valores compactos e $A(t)$ uma família de operadores $m$-acretivos em $X$ satisfazendo $\mathbf{H} 1, \mathbf{H} 2$ e H7. Então, existe $\delta>0$ tal que $T_{\delta} B_{x} F E_{\delta}$ tem um ponto fixo em $C([0, \delta] ; X)$.

Prova: Pelo lema $4.12\left(T_{\delta} B_{x} F E_{\delta}\right)$ é contração e pelo lema 4.13 seus valores são compactos. Segue do Teorema de Nadler que $T_{\delta} B_{x} F E_{\delta}$ tem um ponto fixo.

Exemplo 4.17 Uma aplicaçấo $F: X \rightarrow \mathcal{P}(X)$ é chamada USCO se é semicontínua superiormemente e tem valores compactos. Os lemas 4.12 e 4.13 dizem que sob aquelas condiçôes mais $F(u)$ compacto, para cada $u \in C(0,1] ; X)$, então a aplicação $\left(T_{\delta} B_{x} F E_{\delta}\right)$ é USCO.

Teorema 4.18 Seja $F$ uma aplicação semicontínua inferiormente de $C([0,1] ; X)$ nas partes não vazias de $C([0,1] ; X)$ que leva subconjuntos limitados em conjuntos uniformente integráveis e com valores $(F u)$ fechados para cada $u \in C([0,1] ; X)$. Se $A(t), 0 \leq t \leq 1$, é uma familia de operadores m-acretivos satisfazendo $\mathbf{H 1}, \mathbf{H} 2$ e $\mathbf{H} 7$, então a aplicaçâo

$$
G(u)=\overline{c o}\left(T_{\delta} B_{x} F E_{\delta} u\right)
$$

admite uma seleçâo contínua.

Prova: Pelo Lema $4.13\left(T_{\delta} B_{x} F E_{\delta} u\right)$ é relativamente compacto em $C([0, \delta] ; X)$ e portanto $G(u)$ é compacto. Como $\left(B_{x} F\right)$ é semicontínua inferiormente, então $G$ também o é e assim $G$ é sci e com valores convexos e compactos não vazios. De [43] segue que $G$ admite uma seleção contínua.

Consideremos o seguinte problema: determinar uma função contínua $u$ de $C([0,1] ; X)$ tal que

$$
\left\{\begin{array}{l}
\frac{d u}{d t}+A(t) u(t) \bar{\ni}(F u)(t) \\
u(0)=x \in \overline{D(A(0))}
\end{array}\right.
$$


onde $A(t), 0 \leq t \leq 1$, é uma família de operadores $m$-acretivos e $F$ é uma aplicação de $C([0,1] ; X)$ nas partes não vazias de $C([0,1] ; X)$. Admitiremos sempre $F$ causal.

A equação de inclusão $\left(P_{G}\right)$ deve ser interpretada do seguinte modo: determinar $u \in$ $C([0,1] ; X)$ solução integral de

$$
\left\{\begin{array}{l}
\frac{d u}{d t}+A(t) u(t) \ni f(t) \\
u(0)=x \in \overline{D(A(0))},
\end{array}\right.
$$

para alguma $f \in F u$.

Dizemos que o problema $\left(P_{G}\right)$ acima é viável se $F u \neq \emptyset$ para todo $u$. Um problema viável é chamado normal se $F$ admite uma seleção contínua.

Observamos que toda solução de $\left(P_{G}\right)$ é ponto fixo de $B_{x} F$ e que todo ponto fixo de $B_{x} F$ é solução de $\left(P_{G}\right)$. Uma solução local de $\left(P_{G}\right)$ é qualquer ponto fixo de $\left(T_{a} B_{x} F E_{a}\right)$, para algum $a \in(0,1]$.

Exemplo 4.19 1) Se $F u \equiv f \in C([0,1] ; X)$ para todo $u \in C([0,1] ; X)$, entâo o problema $\left(P_{G}\right)$ é normal e admite uma solução. Ver Teorema 2.7.

2) Se $F$ é unívoca e contínua, o problema $\left(P_{G}\right)$ é normal.

3) Se F é semicontínua inferiormente com valores convexos, fechados e nâo vazios, entâo o problema $\left(P_{G}\right)$ é normal. Veja [43].

4) Se F é semicontínua inferiormente com valores convexos e compactos não vazios, entâo o problema $\left(P_{G}\right)$ é normal.

Se a família de operadores $A(t)$ é unívoca, então o problema acima poder ser reescrito como

$$
\left\{\begin{array}{l}
\frac{d u}{d t}+A(t) u(t) \in(F u)(t) \\
u(0)=x \in \overline{D(A(0))}
\end{array}\right.
$$

que é comum aparecer em teoria do controle, veja [1].

Uma consequência imediata é o seguinte

Teorema 4.20 Seja $A(t), 0 \leq t \leq 1$, uma familia de operadores $m$-acretivos em $X$ satisfazendo $\mathbf{H 1}$ e $\mathbf{H 2}$. Sejam $F$ uma aplicação de $C([0,1] ; X)$ nas partes não vazias de $C([0,1] ; X)$ e $g: C([0,1] ; X) \rightarrow \mathbf{R} \cup\{\infty\}$ semicontínua inferiormente, própria e limitada inferiormente. Se $\left(B_{x} F\right)$ satisfaz à condição

$$
\|u-v\|_{\infty}+g(v) \leq g(u),
$$

para cada $u \in C([0,1] ; X)$ e $v \in\left(B_{x} F\right)$ u, então o problema $\left(P_{G}\right)$ tem uma soluçấo.

Prova: É imediata do Teorema de ponto fixo de Caristi.

Teorema 4.21 Sejam $A(t), 0 \leq t \leq 1$ uma familia de operadores m-acretivos em $X$ satisfazendo $\mathbf{H 1}$ e $\mathbf{H} 2$ e $F$ uma contração de $C([0,1] ; X)$ em $\mathcal{P}(C([0,1] ; X))$ que leva subconjuntos limitados em subconjuntos uniformemente integráveis com $F u$ compacto nâo vazio para todo $u \in C([0,1] ; X)$. Então, o problema $\left(P_{G}\right)$ tem uma solução local. 
Prova: Como na Proposição 4.16, dado $\varepsilon>0$ e $u_{0}$ a solução integral de $P A(t) C(0, x)$, existe um $\delta>0$ tal que $\left(T_{\delta} B_{x} F E_{\delta}\right) C_{\varepsilon}^{\delta} \subset C_{\varepsilon}^{\delta}$. Segue que $\left(T_{\delta} B_{x} F E_{\delta}\right)$ tem um ponto fixo.

Uma consequência imediata decorre do Teorema 3.5.

Corolário 4.22 Seja $F$ como no teorema anterior. Suponha que Fu e h sejam contínuas e de variação limitada e $x \in \hat{D}(A(0))$. Então, a solução local é Lipschitz contínua.

Prova: Como existe $u \in\left(T_{\delta} B_{x} F E_{\delta}\right) u$, então existe $f$ de variação limitada em $[0, \delta]$ tal que $u=T_{\delta} B_{x} f$. Pelo Teorema $3.18 u$ é necessariamente Lipschitz contínua. $\square$ 


\section{Capítulo 5}

\section{Solução de um problema elíptico}

Neste capítulo vamos provar a existência de duas soluções não triviais para um problema elíptico semi-linear

$$
-\Delta u=\lambda_{1} u+g(x, u) \text { em } \Omega \quad u=0 \text { em } \partial \Omega .
$$

No primeiro parágrafo apresentamos o problema e enunciamos o principal resultado, no segundo falamos dos resultados auxiliares que usaremos e finalmente no terceiro parágrafo daremos a prova do teorema principal.

\subsection{Preliminares}

Considere o seguinte problema elíptico

$$
\left\{\begin{array}{l}
-\Delta u=\lambda_{1} u+g(x, u) \text { em } \Omega \\
u=0 \text { em } \partial \Omega
\end{array}\right.
$$

onde $\Omega$ é um domínio limitado em $\mathbf{R}^{N}$ com fronteira $\partial \Omega$ suave, $\lambda_{1}$ é o primeiro autovalor de $\left(-\Delta, H_{0}^{1}(\Omega)\right)$ e $g: \Omega \times \mathbf{R} \longrightarrow \mathbf{R}$ é uma função limitada e de Carathéodory, isto é, $g$ satisfaz as seguintes condiçòes:

a) $g(., s)$ é mensurável em $\Omega$ para quase todo $s \in \mathbf{R}$ fixado,

b) $g(x,$.$) é contínua em \mathbf{R}$ para quase todo $x \in \Omega$.

Assumiremos também que

$$
g(x, u) \rightarrow 0 \text { quando }|u| \rightarrow \infty
$$

uniformemente em $x \in \Omega$ e que existe $k \in L^{\infty}(\Omega)$ tal que

$$
|G(x, u)| \leq k(x), \forall u \in \mathbf{R}, \text { a. e. em } \Omega,
$$

onde $G(x, u)=\int_{0}^{u} g(x, s) d s$.

As condições (2) e (3) caracterizam (1) como um problema de ressonância forte no infinito. Este tipo de problema foi primeiramente estudado por Thews [58] e mais tarde caracterizado como um problema de ressonância forte por Bartolo-Benci-Fortunato [4]. Veja [4] e [55] para uma discussão mais completa e outras referências. 
Estudaremos (1) via métodos variacionais e para isto consideramos o seguinte funcional associado ao problema

$$
F(u)=\frac{1}{2} \int_{\Omega}\left(|\nabla u|^{2}-\lambda_{1} u^{2}\right) d x-\int_{\Omega} G(x, u(x)) d x,
$$

em $H_{0}^{1}(\Omega)$ e procuramos seus pontos críticos. Como $g$ é função limitada e de Carathéodory, então $F$ está bem definido, $F \in C^{1}\left(H_{0}^{1}(\Omega), \mathbf{R}\right)$ e

$$
F^{\prime}(u) h=\int_{\Omega}\left[\nabla u \cdot \nabla h-\lambda_{1} u h-g(x, u) h\right] d x
$$

para todo $u, h \in H_{0}^{1}(\Omega)$. Segue que $u$ é solução (fraca) de (1) se, e somente se, $u$ é ponto crítico de $F$. Veja [22] e [9]. Como usual $H_{0}^{1}(\Omega)$ denota o espaço de Sobolev com a norma $\|u\|=\left(\int_{\Omega}|\nabla u|^{2} d x\right)^{\frac{1}{2}}$, e para $p \geq 1,\|\cdot\|_{p}$ denota a norma em $L^{p}(\Omega)$.

Como $G$ é limitada é fácil ver que $F$ é limitada inferiormente. Temos também que $F$ é não coercivo, pois se $t \in \mathbf{R}$ e se $\varphi_{1}$ é a autofunção associada a $\lambda_{1}$, então

$$
F\left(t \varphi_{1}\right)=-\int_{\Omega} G\left(x, t \varphi_{1}(x)\right) d x
$$

é finito. Portanto $F$ não satisfaz à condição de compacidade de Palais-Smale, pois neste caso, $F$ seria coercivo. Veja [39] e [9].

Lembramos que dados $X$ um espaço de Banach, $A \subset X$ um conjunto fechado com interior não vazio e $\Phi: X \rightarrow \mathbf{R}$ um funcional de classe $C^{1}$, dizemos que $\Phi$ satisfaz a condição de Palais-Smale em $A$ no nível $c \in \mathbf{R},(P S)_{c, A}$ como usualmente representado, se qualquer sequência $\left(u_{n}\right)$ de $A$ tal que $\Phi\left(u_{n}\right) \rightarrow c$ e $\Phi^{\prime}\left(u_{n}\right) \rightarrow 0$ admite subsequência convergente. Se $(P S)_{c, A}$ vale para todo $c \in \mathbf{R}$, então dizemos que $\Phi$ satisfaz a condição de Palais-Smale em $A$. Quando $A=X$ omitimos $A$ nas abreviações e dizemos simplesmente a condiçâo de Palais-Smale no nível $c,(P S)_{c}$, ou condição de Palais-Smale, $(P S)$.

Observação 5.1 Dizemos que $\Phi$ satisfaz a condição (PSB) se satisfaz a condição de PalaisSmale para sequências limitadas. Esta condição é verificada por F definida em (4) para uma grande classe de perturbações $\mathrm{g}$. Por exemplo, se $\mathrm{g}$ tem crescimento subcrítico no infinito, entâo $\nabla F$ é uma perturbação compacta da identidade em $H_{0}^{1}(\Omega)$, e portanto satisfaz (PSB). Veja [51] apêndice $B$.

Admitiremos também que

$$
g(x, 0)=0, \forall x \in \Omega .
$$

Isto implica que $u=0$ é uma solução (trivial) do problema (1) e assim procuramos pontos críticos não nulos de $F$. Por $\lambda_{n}$ representamos o $n$-ésimo valor próprio de $\left(-\Delta, H_{0}^{1}(\Omega)\right)$.

O principal resultado desta seç̧ão é o seguinte:

Teorema 5.2 Assuma que valem as condições (2),(3),(5). Assuma também que existem $\alpha, \beta \in L^{\infty}(\Omega)$, tais que

$$
0 \leq \alpha(x)=\liminf _{u \rightarrow 0} \frac{2 G(x, u)}{u^{2}} \leq \limsup _{u \rightarrow 0} \frac{2 G(x, u)}{u^{2}}=\beta(x)<\lambda_{2}-\lambda_{1},
$$


uniformemente em $x \in \Omega$, com a desigualdade estrita $\alpha(x)>0$ valendo em algum subconjunto de $\Omega$ com medida positiva e que

$$
\int_{\Omega} \lim _{|u| \rightarrow \infty} G(x, u) d x=0
$$

Entâo, o problema (1) tem pelo menos duas soluçôes nâo-triviais.

Resultados de multiplicidade para problemas de ressonância forte foram obtidos por vários autores, por exemplo, [58] e [4]; mas com alguma condição de simetria. Nào assumimos no teorema 5.2 qualquer condição de simetria e nem a condição $(P S)_{c}$, para todo $c \in \mathbf{R}$.

Para a prova deste resultado, que daremos na terceira secção, precisaremos de alguns resultados auxiliares.

\subsection{Resultados auxiliares}

Sejam $X$ um espaço de Banach real e $\Phi: X \rightarrow \mathbf{R}$ um funcional de classe $C^{1}$. Por $K_{c}$ denotaremos o conjunto

$$
K_{c}=\left\{u \in X ; \Phi(u)=c \text { e } \Phi^{\prime}(u)=0\right\}
$$

e por $\Phi^{c}$ o conjunto

$$
\Phi^{c}=\{u \in X ; \Phi(u) \leq c\} .
$$

O seguinte resultado é uma versão $C^{1}$ do Lema da Deformação.

Uma prova completa pode ser encontrada em [52].

Lema 5.3 (Lema de deformação 2) Dados constantes $a<b$, suponha que $\Phi$ não tem valores críticos no intervalo $(a, b)$ e que $\Phi^{-1}(a)$ contém no máximo um número finito de pontos críticos de $\Phi$. Se $\Phi$ satisfaz $(P S)_{c}$ para todo $c \in[a, b)$, então existe uma homotopia $\Phi$-decrescente de homeomorfismos

$$
h:[0,1] \times \Phi^{b} \backslash K_{b} \rightarrow X
$$

tal que

$$
\begin{gathered}
h(0, u)=u \quad \forall u \in \Phi^{b} \backslash K_{b}, \\
h\left(1, \Phi^{b} \backslash K_{b}\right) \subset \Phi^{a} \text { e } h(t, u)=u, \forall u \in \Phi^{a} .
\end{gathered}
$$

O seguinte princípio minimax, devido a Brezis, está relacionado ao lema do passo da montanha generalizado de Rabinowitz. Veja [51], [22], [9] ou [52]. É a principal ferramenta usada na prova do Teorema 5.2. Veja também o Teorema 5.9.

Teorema 5.4 Sejam $X$ um espaço de Banach e $\Phi: X \rightarrow \mathbf{R}$ um funcional $C^{1}$. Seja $Q \subset X$ compacto com bordo $\partial Q$ e $\gamma_{0}: \partial Q \rightarrow X$ uma aplicaçâo contínua. Defina

$$
\Gamma=\left\{\gamma \in C(Q ; X) ; \gamma=\gamma_{0} \quad \text { em } \quad \partial Q\right\}
$$


e seja

$$
c=\inf _{\gamma \in \Gamma} \max _{u \in Q} \Phi(\gamma(u))
$$

Se

$$
\max _{u \in Q} \Phi(\gamma(u))>\max _{u \in \partial Q} \Phi(\gamma(u)), \quad \forall \gamma \in \Gamma,
$$

então existe uma sequência $\left(u_{n}\right)$ tal que $\Phi\left(u_{n}\right) \rightarrow$ e e $\Phi^{\prime}\left(u_{n}\right) \rightarrow 0$.

Em particular, se vale $(P S)_{c}$ então c é um valor crítico de $\Phi$.

Uma situação típica onde o teorema acima é aplicável é quando existe $S \subset X$ não vazio e disjunto de $\partial Q$ e $\gamma_{0}: Q \rightarrow X$ tal que $\gamma_{0}(Q) \cap S \neq \emptyset$, isto é, $\gamma_{0}(\partial Q)$ e $S$ link, e

$$
\sup _{u \in \partial Q} F\left(\gamma_{0}(u)\right)<\inf _{u \in S} F(u) \text {. }
$$

Neste caso, $(*)$ é imediatamente verificada. Além disso, se vale $(P S)_{c}$ então $c$ é um valor crítico. Veja [9].

Outro resultado que será útil na prova do teorema 5.2 é a seguinte situação de linking. A prova pode ser encontrada em [9].

Teorema 5.5 Suponha $X=V \oplus W \operatorname{com} V$ de dimensão finita e e $\in W$ um vetor unitário fixado. Seja

$$
Q=\{u=v+t e, v \in V, t \geq 0 e\|u\| \leq R\}
$$

onde $R>0$ está fixado. Seja $\gamma_{0}: Q \rightarrow X$ uma aplicação contínua tal que

$$
\gamma_{0}(v)=v, \forall v \in V e\|v\| \leq R
$$

e que para algum $r>0$

$$
\left\|\gamma_{0}(u)\right\| \geq r, \forall u \in Q \text { e }\|u\|=R .
$$

Então, $\gamma_{0}(Q) \cap S \neq \emptyset$, para todo conjunto

$$
S=\{w \in W ;\|w\|=\rho, 0<\rho<r\} .
$$

Um resultado já conhecido é o seguinte [9].

Teorema 5.6 Suponha que $\Phi$ seja limitado inferiormente e de classe $C^{1}$. Se vale $(P S)_{c}$ com $c=\inf _{X} \Phi$, então $\Phi$ assume o seu mínimo.

A prova é simples. Suponha $K_{c}=\emptyset$, então pelo $1^{-0}$ lema da deformação existem $\varepsilon>0$ e homotopia $h$ tal que $\emptyset \neq h\left(1, \Phi^{c+\varepsilon}\right) \subset \Phi^{c-\varepsilon}=\emptyset$, absurdo. 


\subsection{Prova do teorema principal}

Nesta secção provaremos a existência de duas soluções não triviais para o problema elíptico de ressonância forte (1). Precisaremos de mais um resultado auxiliar.

O seguinte lema é uma situação especial de um resultado provado em [55]; daremos aqui uma prova diferente.

Lema 5.7 Assuma as condições (2), (3) e (7). Então, $F$ satisfaz $(P S)_{c}$, para todo c $\neq 0$.

Prova:Como $g$ tem crescimento subcrítico no infinito então $\nabla F$ é uma perturbação compacta da identidade, veja [51] apêndice B ou [22]. Logo, basta provar que toda sequência $\left(u_{n}\right)$ satisfazendo

$$
F\left(u_{n}\right) \rightarrow c \neq 0 \text { e } F^{\prime}\left(u_{n}\right) \rightarrow 0
$$

é necessariamente limitada.

Sejam $V=\operatorname{Span}\left\{\varphi_{1}\right\}$ e $W=V^{\perp} \cap H_{0}^{1}(\Omega)$, onde $\varphi_{1}>0$ é a $\lambda_{1}$-autofunção normalizada de $\left(-\Delta, H_{0}^{1}(\Omega)\right)$, com ortogonalidade no sentido $L^{2}$, então podemos escrever

$$
H_{0}^{1}(\Omega)=V \oplus W
$$

$u_{n}=t_{n} \varphi_{1}+w_{n} \in H_{0}^{1}(\Omega)$ de modo único.

Temos que

$$
F^{\prime}\left(u_{n}\right) w_{n}=\left\|w_{n}\right\|^{2}-\lambda_{1}\left\|w_{n}\right\|_{2}^{2}-\int_{\Omega} g\left(x, u_{n}(x)\right) w_{n} d x=o\left(\left\|w_{n}\right\|\right)
$$

e da caracterização variacional do segundo valor próprio $\lambda_{2}$ de $\left(-\Delta, H_{0}^{1}(\Omega)\right)$, podemos escrever

$$
\frac{\lambda_{2}-\lambda_{1}}{\lambda_{2}}\left\|w_{n}\right\|^{2} \leq \int_{\Omega} g\left(x, u_{n}(x)\right) w_{n}(x) d x+o\left(\left\|w_{n}\right\|\right) .
$$

Como $g$ é limitada e $w_{n} \in H_{0}^{1}(\Omega)$, então $\left(\left\|w_{n}\right\|\right)$ é uma sequência limitada.

Então, se $\left(\left\|u_{n}\right\|\right)$ não é limitada, necessariamente $\left|t_{n}\right| \rightarrow \infty$. Como existe subsequência de $\left(w_{n}\right)$, ainda denotada por $\left(w_{n}\right)$, que converge na topologia fraca para algum $w \in H_{0}^{1}(\Omega)$, então da inclusão compacta de $H_{0}^{1}(\Omega)$ em $L^{2}(\Omega)$, temos que $w_{n} \rightarrow w$ em $L^{2}(\Omega)$. Passando a uma subsequência se necessário, podemos supor que $w_{n}(x) \rightarrow w(x)$ para quase todo $x \in \Omega$. Isto implica com (2) que $g\left(x, u_{n}(x)\right) \rightarrow 0$ para quase todo $x \in \Omega$. Logo, temos que

$$
\begin{aligned}
\frac{\lambda_{2}-\lambda_{1}}{\lambda_{2}} \lim _{n \rightarrow \infty}\left\|w_{n}\right\|^{2} & \leq \lim _{n \rightarrow \infty} \int_{\Omega} g\left(x, u_{n}(x)\right) w_{n}(x) d x \\
& \leq \lim _{n \rightarrow \infty}\left(\int_{\Omega} g^{2}\left(x, u_{n}(x)\right) d x\right)^{\frac{1}{2}}\left\|w_{n}\right\|_{2}^{2} .
\end{aligned}
$$

Como $g_{n}(x)=g^{2}\left(x, u_{n}(x)\right)$ é mensurável e limitada, segue do teorema da convergência dominada de Lebesgue que

$$
\lim _{n \rightarrow \infty}\left\|w_{n}\right\|=0
$$


Finalmente usando (7) e ortogonalidade temos de

$$
F\left(u_{n}\right)=\frac{1}{2}\left\|w_{n}\right\|^{2}-\lambda_{1}\left\|w_{n}\right\|_{2}^{2}-\int_{\Omega} G\left(x, u_{n}(x)\right) d x
$$

que

$$
\lim _{n \rightarrow \infty} F\left(u_{n}\right)=0 \text {, }
$$

o que é absurdo pois $F\left(u_{n}\right) \rightarrow c \neq 0$. Logo, $F$ satisfaz $(P S)_{c}$, para todo $c \neq 0$.

Novamente, observamos que $(P S)_{0}$ não pode valer, pois isto mais o lema anterior implicariam que $F$ é coercivo, o que é absurdo.

Agora estamos em condições de dar a prova do teorema principal.

\section{Prova do teorema 5.2}

De (6) segue que dado $\varepsilon>0$ existe $\delta>0$ tal que se $|u|<\delta$, então

$$
(\alpha(x)-\varepsilon) u^{2} \leq 2 G(x, u) \leq(\beta(x)+\varepsilon) u^{2} \forall x \in \Omega .
$$

Como $\alpha>0$ em algum subconjunto de medida positiva e $\varphi_{1}>0$, segue que para $\varepsilon>0$ suficientemente pequeno

$$
\int_{\Omega}(\alpha(x)-\varepsilon) \varphi_{1}^{2}(x) d x=2 K>0
$$

e portanto para $v \in V$, como $|v(x)|=|t| \varphi_{1}(x)$ e $\|v\|=|t|$, se

$$
\|v\| \leq \frac{\delta}{\left\|\varphi_{1}\right\|_{\infty}}=R
$$

temos

$$
\begin{aligned}
F(v)=-\int_{\Omega} G(x, v(x)) d x & \leq-\frac{1}{2} \int_{\Omega}(\alpha(x)-\varepsilon) v^{2}(x) d x \\
& =-\frac{1}{2} \int_{\Omega}(\alpha(x)-\varepsilon) \varphi_{1}^{2}(x)\|v\|^{2} d x \\
& =-\frac{1}{2} 2 K\|v\|^{2} \\
& =-K\|v\|^{2} .
\end{aligned}
$$

Isto mostra que existe $R>0$ tal que $F(v)<0$ em

$$
B_{V}(0, R)=\left\{s \varphi_{1} ;|s| \leq R\right\}
$$

e como $F$ é limitado inferiormente, $l=\inf _{H_{0}^{1}(\Omega)} F<0$. Agora do lema 5.7, $F$ satisfaz a condição $(P S)_{c}$ para $c<0$ e portanto seu ínfimo é assumido, digamos em $u_{0} \in H_{0}^{1}(\Omega)$. Assim encontramos um valor crítico negativo para $F$.

Agora para encontrar o segundo ponto crítico não nulo de $F$, vamos assumir que não existem valores críticos no intervalo $(l, 0)$ e que $u_{0}$ e 0 sejam os úncios pontos críticos de $F$, 
pois caso contrário a prova está terminada. Vamos aplicar o teorema 5.4 para mostrar que existe um valor crítico em $(0, \infty)$. Fixemos um ponto $e \in W$ unitário e defina o seguinte conjunto

$$
Q=\left\{u=s \varphi_{1}+t e ;|s| \leq R, t \geq 0,\|u\| \leq R\right\} .
$$

Segue que $Q$ é subconjunto compacto de $H_{0}^{1}(\Omega)$ e tem fronteira (relativo a $V \oplus \mathbf{R} e$ )

$$
\partial Q=\left(B_{V}(0, R) \times\{0\}\right) \cup\{u \in Q,\|u\|=R\} .
$$

Como não existem valores críticos em $(l, 0)$, existe pelo lema da deformação uma homotopia de homeomorfismos $F$-decrescente $h:[0,1] \times F^{0} \backslash\{0\} \rightarrow H_{0}^{1}(\Omega)$ tal que $h(1, u)=\left\{u_{0}\right\}$. Assim podemos definir $\tilde{\gamma}: B_{V}(0, R) \rightarrow H_{0}^{1}(\Omega)$ por

$$
\tilde{\gamma}\left(s \varphi_{1}\right)=\left\{\begin{array}{lll}
u_{0} & \text { se } & |s|<R / 2 \\
h\left(\frac{2(R-|s|)}{R}, \frac{R s \varphi_{1}}{|s|}\right) & \text { se } & |s| \geq R / 2 .
\end{array}\right.
$$

É claro que $\tilde{\gamma}$ é contínua em $B_{V}(0, R)$, e como $h$ é $F$-decrescente, isto é, $F\left(h\left(s_{1}, u\right)\right)>$ $F\left(h\left(s_{2}, u\right)\right)$ se $0 \leq s_{1}<s_{2} \leq 1$, temos

$$
F(\tilde{\gamma}(v)) \leq \max _{\partial B_{V}(0, R)} F=d<0
$$

para todo $v \in B_{V}(0, R)$ com $\|v\| \geq \frac{R}{2}$. A seguir definimos uma aplicação $\gamma_{0}: \partial Q \rightarrow H_{0}^{1}(\Omega)$ por

$$
\gamma_{0}\left(s \varphi_{1}+t e\right)=\left\{\begin{array}{lll}
s \varphi_{1} & \text { se } & t=0 \\
\tilde{\gamma}\left(s \varphi_{1}\right) & \text { se } & 0<t \leq R, 0 \leq|s|<R .
\end{array}\right.
$$

É fácil ver que $\gamma_{0}$ é também contínua pois é uma projeção ou uma composta de contínuas, além disso pode ser continuamente extendida para todo o conjunto $Q$. Para completar as hipóteses da condição de linking devemos provar que, para algum $r>0,\left\|\gamma_{0}(u)\right\| \geq r$ para todo $u \in \partial Q$ tal que $\|u\|=R$. De fato, como $F(0)=0$ segue da continuidade que dado $0<\varepsilon<-d$, existe $r>0$ tal que se $\|u\| \leq r$ então $F(u) \geq-\varepsilon>d$. Assim, se $F(u) \leq d<0$, então temos $\|u\| \geq r$. Portanto de (11) segue que $\|\tilde{\gamma}(u)\| \geq r$ para todo $u \mathrm{em}$ $\{u \in \bar{\partial} Q:\|u\|=R\}$, pois $\tilde{\gamma}\left(B_{V}\right)=\gamma_{0}(\{u \in \partial Q:\|u\|=R\})$, e a condição vale. Então do Teorema de linking, $\gamma(Q) \cap S \neq \emptyset$ para todo conjunto

$$
S=\{w \in W ;\|w\|=\rho, 0<\rho<r\},
$$

onde

$$
\Gamma=\left\{\gamma \in C\left(Q, H_{0}^{1}(\Omega)\right) ; \gamma=\gamma_{0} \text { em } \partial Q\right\}
$$

Para terminar a prova mostraremos que existe um conjunto $S$ tal que $\inf _{S} F>0$. Isto implicará que a condição do princípio minimax vale para $Q$ e $S$, e assim

$$
c=\inf _{\gamma \in \Gamma} \max _{u \in Q} F(\gamma(u))>0
$$


é um valor crítico de $F$ pois $F$ satisfaz $(P S)_{c}$ para $c>0$. De fato, como $G$ é uniformemente limitada, dado $\delta>0$ existe uma constante $C_{\delta}>0$ e $\sigma>2 \operatorname{com} \sigma=\frac{2 N}{N-2}$ se $N \geq 3$ e $\sigma=3$ se $N=1,2$ tal que

$$
|G(x, u)| \leq C_{\delta}|u|^{\sigma}, \quad|u| \geq \delta
$$

para quase todo $x \in \Omega$. Combinando a desigualdade acima com (10) temos que para todo $u \in \mathbf{R}$, e para quase todo $x \in \Omega$,

$$
G(x, u) \leq \frac{1}{2}(\beta(x)+\varepsilon) u^{2}+C_{\delta}|u|^{\sigma} .
$$

Como $\beta(x)<\lambda_{2}-\lambda_{1}$, temos (para $\varepsilon$ suficientemente pequeno)

$$
\int_{\Omega}(\beta(x)+\varepsilon) u^{2} \leq\left(\lambda_{2}-\lambda_{1}-\varepsilon\right) \int_{\Omega} u^{2}
$$

Então para todo $w \in W$,

$$
\begin{aligned}
F(w) & \geq \frac{1}{2} \int_{\Omega}|\nabla w|^{2}-\frac{\lambda_{1}}{2} \int_{\Omega}(\beta(x)+\varepsilon) w^{2}-C_{\delta} \int_{\Omega}|w|^{\sigma} \\
& \geq \frac{1}{2}\|w\|^{2}-\frac{1}{2}\left(\lambda_{2}-\varepsilon\right) \int_{\Omega} w^{2}-C_{\delta}\|w\|_{\sigma}^{\sigma} \\
& \geq \frac{\varepsilon}{2 \lambda_{2}}\|w\|^{2}-C_{\delta} \theta\|w\|^{\sigma}
\end{aligned}
$$

onde $\theta$ é a constante de Sobolev do mergulho de $H_{0}^{1} \subset L^{\sigma}$. Então, como $\sigma>2$, segue que $F(w)>0$ em $S=\{w \in W ;\|w\|=\rho\}$ com $0<\rho<r$ suficientemente pequeno.

Observação 5.8 O Teorema 5.2 continua válido se a condição (6) for substituída por

$$
\lambda_{k}-\lambda_{1}<\alpha \leq \liminf _{u \rightarrow 0} \frac{2 G(x, u)}{u^{2}} \leq \limsup _{u \rightarrow 0} \frac{2 G(x, u)}{u^{2}} \leq \beta<\lambda_{k+1}-\lambda_{1},
$$

para algum $k \geq 1$ natural.

Observamos que os extremos de (6) são obtidos de $\left(6^{\prime}\right)$ tomando $k=1$. A prova é essencialmente a mesma com $H_{0}^{1}(\Omega)$ decomposto como $V_{k} \oplus W_{k}$, onde $V_{k}=\operatorname{Span}\left\{\varphi_{1}, \ldots, \varphi_{k}\right\}$ e $W_{k}=V_{k}^{\perp} \cap H_{0}^{1}(\Omega)$, ortogonalidade no sentido de $L^{2}$.

O seguinte teorema mostra que o conjunto $\Gamma$ é não vazio. A demonstração pode ser encontrada no livro de Spanier, Algebraic Topology, McGraw-Hill, New York (1966).

Teorema 5.9 Sejam E um espaço de Banach e $C$ um subconjunto fechado de um espaço métrico $X$. Toda aplicação contínua $f: C \rightarrow E$ tem uma extensão contínua $F: X \rightarrow$ $c o(f(C))$ cuja restriçâo a $X \backslash C$ é localmente lipschitziana.

Veremos a seguir um exemplo simples relacionado ao problema anterior. 
Exemplo 5.10 Consideremos como caso particular de Au $\ni f$ o seguinte problema

$$
\left\{\begin{array}{l}
-\Delta u=\lambda_{1} u+g(x, u) \text { em } \Omega \\
u(0)=0 \text { em } \partial \Omega
\end{array}\right.
$$

onde $\Omega \subset \mathbf{R}^{n}, \lambda_{1}$ é o primeiro autovalor de $-\Delta$ em $X=H_{0}^{1}(\Omega)$ e $g: \Omega \times \mathbf{R} \rightarrow \mathbf{R}$ é uma função limitada e Carathéodory tal que

$$
g(x, 0)=0,
$$

para todo $x \in \Omega$.

Associado ao problema acima consideremos o seguinte funcional $F: H_{0}^{1}(\Omega) \rightarrow \mathbf{R}$ dado por

$$
F(u)=\frac{1}{2} \int_{\Omega}\left(|\nabla u|^{2}-\lambda_{1} u^{2}\right) d x-\int_{\Omega} G(x, u(x)) d x
$$

onde

$$
G(x, s)=\int_{0}^{s} g(x, t) d t
$$

é limitada por alguma constante $K>0$. É fácil ver que $F$ é de classe $C^{1}$, limitado inferiormente $e$,

$$
F(0)=0
$$

e que as soluções (fracas) do problema acima são precisamente os pontos críticos de $F$. Admita que

$$
\int_{\Omega} \lim _{|s| \rightarrow \infty} G(x, s) d x=0
$$

que

$$
\text { existe } r>0 \text { tal que }-\infty<\inf _{X} F(u)<\inf _{\partial B(0, r)} F=a
$$

e que

$$
g(x, s) \rightarrow 0 \text { quando }|s| \rightarrow \infty .
$$

As condições (4), (6),(8) implicam que $F$ satisfaz $(P S)_{c}, \forall c \neq 0$, enquanto que as condiçóes $(4),(8)$ caracterizam, como já vimos, o problema como um problema de ressonância forte no infinito. Veja [9].

Observamos que $u=0$ é uma solução de (1). Afirmamos que nas condiçôes acima o problema (1) tem pelo menos uma solução não trivial. De fato, seja $A=X-B(0, r)$. Temos

a) $c=\inf _{A} F>-\infty$

b) $\exists w \in$ int A tal que $F(w)<\inf _{\partial A} F$.

c) vale $(P S)_{c, A}$, para $c=\inf _{A} F$, pois dado uma sequência minimizante $\left(u_{n}\right)$ como inf $\cos _{X} F$ a existe $n_{0} \in \mathbf{N}$ tal que $u_{n} \notin B(0, r)$ e portanto $u_{n} \in A$ para todo $n \geq n_{0}$.

Portanto, estão verificadas as condições do seguinte teorema: 
Teorema 5.11 ([Mizoguchi] [45]) Seja $F \in C^{1}(X ; \mathbf{R})$ limitado inferiormente sobre um subconjunto fechado $A \subset X$ com interior não vazio. Suponha também que

$$
F(\tilde{u})<\inf _{\partial A} F \text { para algum } \tilde{u} \in \text { int } A .
$$

Se $F$ satisfaz $(P S)_{c, A}$, então $F$ tem um mínimo local $u \in$ int $A$.

Segue do teorema de Mizoguchi que F tem um minimo local no interior de A. Portanto, o problema (1) tem uma solução nâo trivial. 


\section{Referências}

[1] N. U. Ahmed. Nonlinear Analysis and Applications. Lectures Note in Pure and Applied Maths no.109,39-49 (1991).

[2] G. Auchmuty. Variational Principles for Operator Equations and Initial Value Problems. Nonlinear Analysis, Theory, Methods and Applications, vol 12, no. 5, 531-564 (1988).

[3] V. Barbu. Nonlinear Semigroups and Differential Equations in Banach Spaces. Nordoff Inst. Publishing (1976).

[4] P. Bartolo, V. Benci and D. Fortunato. Abstract critical point theorems and applications to some nonlinear problems with strong resonance at infinity. Nonl. Anal. TMA 7 (1983), 981-1012.

[5] B. Beauzamy. Introduction to Banach spaces and their Geometry. North-Holland Mathematics Studies (1982).

[6] Ph. Bénilan. Equations d'Evolution dans un Space de Banach Quelconque et applications. These, Orsay (1972).

[7] H. Brezis. New Results Concerning Monotone Operators and Nonlinear Semigroups. R. I. M. S., Kyoto Univ. (1975).

[8] H. Brezis. Analyse Fonctionnelle- Thèorie et Applications. Masson (1987).

[9] H. Brezis and L. Nirenberg. Remarks on finding critical points. Comm. Pure App. Math. 44 (1991), 939-963.

[10] H. Brezis and Pazy. Accretive Sets and Differential Equations in Banach Spaces. Israel J. Math. 8, 367-383 (1970).

[11] F. E. Browder. Nonlinear accretive Operators in Banach Spaces. Bull. Amer. Math. Soc. 73 (1967), 470-476.

[12] A. Capozzi, D. Lupo and S. Solimini. On the existence of a nontrivial solutions to nonlinear problems at resonance. Nonl. Anal. TMA 13 (1989), 151-163.

[13] K. C. Chang, Variational methods for non-differentiable function and their applications to partial differential equations. J. Math. Anal. Appl. 80 (1981), 102-129. 
[14] E. Cramer, Laksmikantham and A. R. Mitchel. On Existence of Weak Solutions of Differential Equations in Non-reflexive Banach Spaces. Nonlinear Analysis, Theory. Methodos and Appl., vol. 2, no. 2, 169-177 (1976).

[15] M. Crandall et Ligget. Generation of Semigroups of Nonlinear Transformation in General Banach Spaces. Am. J. Math., 93, 265-298 (1971).

[16] M. Crandall and Pazy. Nonlinear Evolution Equations in Banach Spaces. Israel J. Math., 11, 57-49 (1972).

[17] F. S. DeBlase. On the Differentiability of Multifunctions. Pacific J. Math., 66, 67-81 (1976).

[18] K. Deimling. Ordinary Differential Equations in Banach Spaces. Lectures Note in Mathematics 596. Springer-Verlag (1977).

[19] J. Diestel and J. J. Ulh Jr. Vector Measures. Mathematics Survey no. 15. Am. Math. Soc., Providence, Rodhe Island (1977).

[20] I. Ekeland and J. P. Aubin. Applied Nonlinear Analysis. John Wiley and Sons (1984).

[21] G. D. Falkner. On the Non Existence of Weak Solutions to Abstract Differential Equations in Nonreflexive Spaces. Nonlinear Analysis, 2, 505-508 (1978).

[22] D. G. Figueiredo. The Ekeland Variational Principle With Applications and Detours. Springer-Verlag (1989).

[23] W. E. Fitzgibbon. Weakly Continuous Accretive Operators in General Banach Spaces. Bull. Austral. Math Soc., vol 41, 185-199 (1990).

[24] A. Fonda and J. P. Gossez. Semicoercive variational problems at ressonance: an approach abstract. Diff. Int. Eq. 3 (1990), 695-708.

[25] S. Gutman. Existence Theorems for Nonlinear Evolution Equations. Nonlinear Analysis, vol 11, no. 10, 1193-1206 (1987).

[26] S. Gutman. Compact Perturbation of m-accretive operators in General Banach Spaces. SIAM J. Math. Anal., 13, 789-800 (1982).

[27] K. Goebel and J. Banaś. Measure of Noncompactness in Banach spaces. Lecture notes in pure and applied mathematics, vol 60, (1980).

[28] E. Hille and R. S. Phillips. Functional Analysis and Semigroups. Amer. Math. Soc., Providence R. I. (1957).

[29] C. S. Hönig. Aplicações da Topologia à Análise. Projeto Euclides, IMPA (1976).

[30] V. I. Istratescu. Strict Convexity and Complex Strict Convexity. Dekker (1984).

[31] V. I. Istratescu. Fixed Points Theory, An Introduction. D. Reidel Publishing Company (1988). 
[32] T. Kato. Nonlinear Semigroups and Evolution Equations. J. Math. Soc. Japon., 19, 508-520 (1967).

[33] D. B. Khang. On The Asymptotic Regularity of Non Expansive Maps. Math. Hung.48 $(1,2), 109-115$ (1986).

[34] Knight. Solutions of Differential Equations in Banach Spaces. Duke Math. J., 41, 437-442 (1974).

[35] K. Kobayasi,Y. Kobayashi and S. Oharu. Nonlinear Evolution Operators in Banach Spaces. Osaka J. Math., 21 281-310 (1984).

[36] Y. Komura. Nonlinear Semigroups in Hilbert Spaces. J. Math. Soc., 19, 493-507 (1967).

[37] K. Kuratowski. Topology, vol. III. Academic Press, New York (1966).

[38] V. Lakshmikanthan and S. Leela. Nonlinear Differential Equations in Abstract Spaces. International Series in Nonlinear Mathematics, Pergamon Press (1981).

[39] S. Li. Some existence theorems of critical points and applications. Preprint ICTP, IC $86 / 90,1986$.

[40] J. L. Lions and E. Magenes. Non Homogeneous Boundary Value Problems and Applications, vol. I. Springer-Verlag (1972).

[41] A. Marino and G. Prodi. Metodi perturbative nella teoria di Morse. Boll. UMI 11 (3) (1975),1-32.

[42] R. H. Martin Jr. Differential Equations on Closed Subsets of a Banach Spaces. Trans. Am. Math Soc. 179, 399-414 (1973).

[43] E. Michael. Continuous Selections. Annals of Math., vol. 63,no 2 march, 361-382 (1956).

[44] N. Mizoguchi. A Generalization of Bronsted Results and its Applications. Proccedings of the American Mathematical Society, vol 108, no. 03 march, 707-714 (1990).

[45] N. Mizoguchi. Existence of a nontrivial solutions of partial differential equations with discontinuous nonlinearities. Nonlinear Analysis, 16, 1025-1034 (1991)

[46] G. Morosanu. Nonlinear evolution equations and applications. D. Reidel Pub. Co. (1988).

[47] N. H. Pavel. Nonlinear Evolution Operators and Semigroups Applications to Partial Differential Equations. Lectures Note in Math. no.1260. Springer-Verlag (1987).

[48] N. H. Pavel. Nonlinear Evolution Equations Governed by f-quasi- dissipative Operators. Nonlinear Analysis. vol 5. no. 5, 449-468 (1981). 
[49] A. Pazy. A class of Semilinear Equations of Evolutions. Israel J. Math. 20, 23-26 (1975).

[50] R. R. Phelps. Convex Functions, Monotone Operators and Differentiability. LNM 1364. Springer-Verlag (1988).

[51] P. Rabinowitz. Minimax methods in critical theory with applications to differential equations. BCMS Reg. Conf. S. Math. 65, AMS, Providence, 1986.

[52] M. Ramos and C. Rebelo. A unified approach to min-max critical points theorems. Portugaliae Math. (to appear).

[53] M. Ramos and L. Sanches. Variational elliptic problems involving noncoercive functionals. Proc. R. Soc. Edinburgh 112A (1989), 177-185.

[54] E. Schechter. Perturbations of Regularizing Maximal Monotone Operators. Israel J. Math. 43, 49-61 (1982).

[55] E. A. B. Silva. Linking theorems and applications to semilinear elliptic equations at resonance. Nonl. Anal. TMA 16 (1991), 455-477.

[56] C. Stegall. Functions of the First Baire Class With Values in Banach Spaces. Proccedings of the Amer. Math. Soc., vol 111, no. 4 april, 981-991 (1991).

[57] A. Szep. Existence Theorem of Weak Solutions of Ordinary Differential Equations in reflexive Banach Spaces. Studia Sci. Math. Hungar., 6,197-203 (1971).

[58] K. Thews. Nontrivial solutions of elliptic equations at resonance. Proc. R. Soc. Edinburgh 85A (1980), 119-129.

[59] L. Vesely. Some new results on Accretive Multivalued Operators. Comment. Math. Univ. Carolinae 30, 1, 45-55 (1989).

[60] D. Volkman. ein Existenzats Sur Gewolneiche Differentialgleichungen in Banach Raumen. Proc. Amer. Math. Soc., 80, 297-300 (1980).

[61] I. Vrabie. The Nonlinear Version of Pazy Local Existence Theorem. Israel J, Math 32, 221-235 (1979).

[62] D. Wagner. Survey of measurable selections theorems. SIAM J. Control Optim., 15 (1977), 859-903.

[63] G. Webb. Continuous Nonlinear Perturbations of Linear Accretive Operators in Banach Spaces. J. Funct. Anal. 10, 191-203 (1972). 